\title{
Projectors and seed conformal blocks for traceless mixed-symmetry tensors
}

\author{
Miguel S. Costa, ${ }^{a, b}$ Tobias Hansen, ${ }^{a, c}$ João Penedones ${ }^{a, b, d}$ and Emilio Trevisani ${ }^{a}$ \\ ${ }^{a}$ Centro de Física do Porto, Departamento de Física e Astronomia, \\ Faculdade de Ciências da Universidade do Porto, \\ Rua do Campo Alegre 687, 4169-007 Porto, Portugal \\ ${ }^{b}$ Theory Division, Department of Physics, CERN, \\ CH-1211 Genève 23, Switzerland \\ ${ }^{c}$ II. Institut für Theoretische Physik, Universität Hamburg, \\ Luruper Chaussee 149, D-22761 Hamburg, Germany \\ ${ }^{d}$ Fields and Strings Laboratory, Institute of Physics, EPFL, \\ CH-1015 Lausanne, Switzerland \\ E-mail: miguelc@fc.up.pt, thansen@fc.up.pt, joao.penedones@epfl.ch, \\ emiliotrevisani@yahoo.it
}

ABSTRACT: In this paper we derive the projectors to all irreducible $\mathrm{SO}(d)$ representations (traceless mixed-symmetry tensors) that appear in the partial wave decomposition of a conformal correlator of four stress-tensors in $d$ dimensions. These projectors are given in a closed form for arbitrary length $l_{1}$ of the first row of the Young diagram. The appearance of Gegenbauer polynomials leads directly to recursion relations in $l_{1}$ for seed conformal blocks. Further results include a differential operator that generates the projectors to traceless mixed-symmetry tensors and the general normalization constant of the shadow operator.

Keywords: Conformal and W Symmetry, Field Theories in Higher Dimensions

ArXiv EPrint: 1603.05551 


\section{Contents}

1 Introduction 2

2 Projectors to traceless mixed-symmetry tensors 3

2.1 Review of projectors to traceless symmetric tensors 3

2.2 Projectors for Young diagrams with two rows 4

2.2.1 Birdtrack notation 6

2.2.2 Projectors to the irreps $\left(l_{1}, 1\right) \quad 6$

$\begin{array}{lll}\text { 2.2.3 Algorithm for solving the differential equations } & 7\end{array}$

2.2.4 Projectors to the irreps $\left(l_{1}, 2\right) \quad 8$

2.3 Projectors for Young diagrams with three rows 8

2.3.1 Projectors to the irreps $\left(l_{1}, 1,1\right) \quad 9$

2.3.2 Projectors to the irreps $\left(l_{1}, 2,1\right) \quad 9$

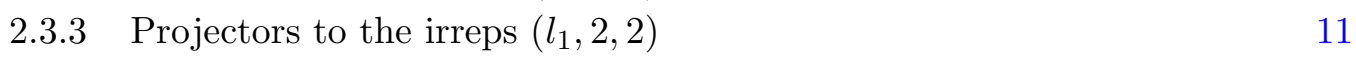

$\begin{array}{ll}2.4 & \text { Normalization of the projectors } \\ \end{array}$

3 Recursion relations for seed conformal blocks $\quad 14$

$\begin{array}{ll}3.1 \text { Classification of seed conformal blocks } & 14\end{array}$

$\begin{array}{lll}3.2 & \text { Three-point functions } & 15\end{array}$

$\begin{array}{ll}3.3 & \text { Recursion relations from the shadow formalism } \\ 3.4 & \text { Solution }\end{array}$

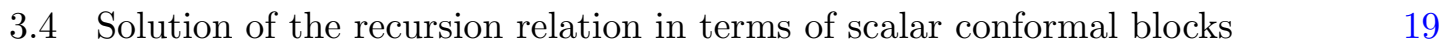

3.5 Recursion relation for radial coordinates 20

4 Concluding remarks $\quad 20$

A A mixed-symmetry differential operator $\quad 22$

B Relating different projectors $\quad 24$

C More projectors $\quad 24$

$\begin{array}{lll}\text { C.1 Projectors to the irreps }\left(l_{1}, 3\right) & 25\end{array}$

C.2 Projectors to the irreps $\left(l_{1}, 4\right) \quad 26$

$\begin{array}{lll}\text { C.3 Projectors to the irreps }\left(l_{1}, 3,1\right) & 28\end{array}$

D Computation of the constants $\mathcal{S}_{\Delta \Delta_{12}}^{\lambda}$ from the shadow formalism $\quad 30$

D.1 Spin transfer operator 31

D.2 Young diagrams with two rows 32

D.3 Arbitrary representations 36

D.4 Conformal integrals 38

$\begin{array}{lll}\text { E OPE limit of conformal blocks in the shadow formalism } & 39\end{array}$

$\begin{array}{llr}\text { F Spherical tensor harmonics } & 41\end{array}$

F.1 Covariant derivatives 43 


\section{Introduction}

One missing ingredient for the conformal bootstrap program [1-34] for correlation functions with operators with spin in $d$ dimensions is the explicit knowledge of seed conformal blocks exchanging mixed-symmetry tensors. Seed conformal blocks are the conformal blocks exchanging a given irreducible representation (irrep) of $\mathrm{SO}(d)$, while having a minimal amount of spin in the external operators, such that the conformal block is unique. They are seeds in the sense that conformal blocks for the exchange of the same representation, but for external operators with higher spin, can be derived from them by acting with differential operators that generate the required extra tensor structures, as described in $[26,35,36]$.

Much of the structure of a seed conformal block is encoded by the projector to the $\mathrm{SO}(d)$ irrep which labels the exchanged operator. This can be seen by considering the integral expressions of the conformal blocks in the shadow formalism [37-39]. Indeed, the lack of explicit expressions for the projectors is the main reason why the seed conformal blocks are still unknown. So far, the projectors and seed conformal blocks in $d$ dimensions are only known for the exchanged operators in the irreps $\left(l_{1}\right)=\square[\ldots \square[38,40,41]$ and $\left(l_{1}, 1\right)=\square[42]$. Expressions for the projectors to the irreps $\left(l_{1}, 1,1\right)$ and $\left(l_{1}, 2\right)$ were given in $[43,44]$. Table 1 shows all irreps that appear in a correlator of four stress-tensors, and for each irrep the correlator where it appears in a seed conformal block.

In section 2 the projectors for all irreps appearing in table 1 will be derived in a compact form. The length of the first row of the Young diagram $l_{1}$ is left unspecified and only appears in the final results as a parameter of Gegenbauer polynomials and in the overall normalization. A consequence of this are universal recursion relations in $l_{1}$ for the seed conformal blocks. These recursion relations are shown to hold for the seed conformal blocks of all the correlators in table 1 and conjectured to hold for any seed conformal block of bosonic operators. These relations are derived in section 3, making use of the integral representations of the conformal blocks in the shadow formalism, where the projector appears explicitly. Section 4 presents final remarks.

The appendices contain several other general results related to projectors and seed conformal blocks. In appendix A we derive a differential operator that generates projectors to traceless mixed-symmetry tensors for Young diagrams of two rows. This operator is a generalization of a well known operator for traceless symmetric tensors. Appendix B deals with a relation between projectors of different irreps that arise from certain index contractions. In appendix $\mathrm{C}$ we state some of the longer explicit results for projectors. Appendix D computes the normalization constant that arises when a shadow transformation is performed on an operator in any three-point function that can appear in a seed conformal block. In appendix E the OPE limit of general seed conformal blocks in the shadow formalism is computed to facilitate comparisons to other results. Finally, in appendix F we explain the relation between projectors to traceless mixed-symmetry tensors and tensor harmonics on the sphere. Included with this work is a Mathematica notebook containing the derived projectors. 


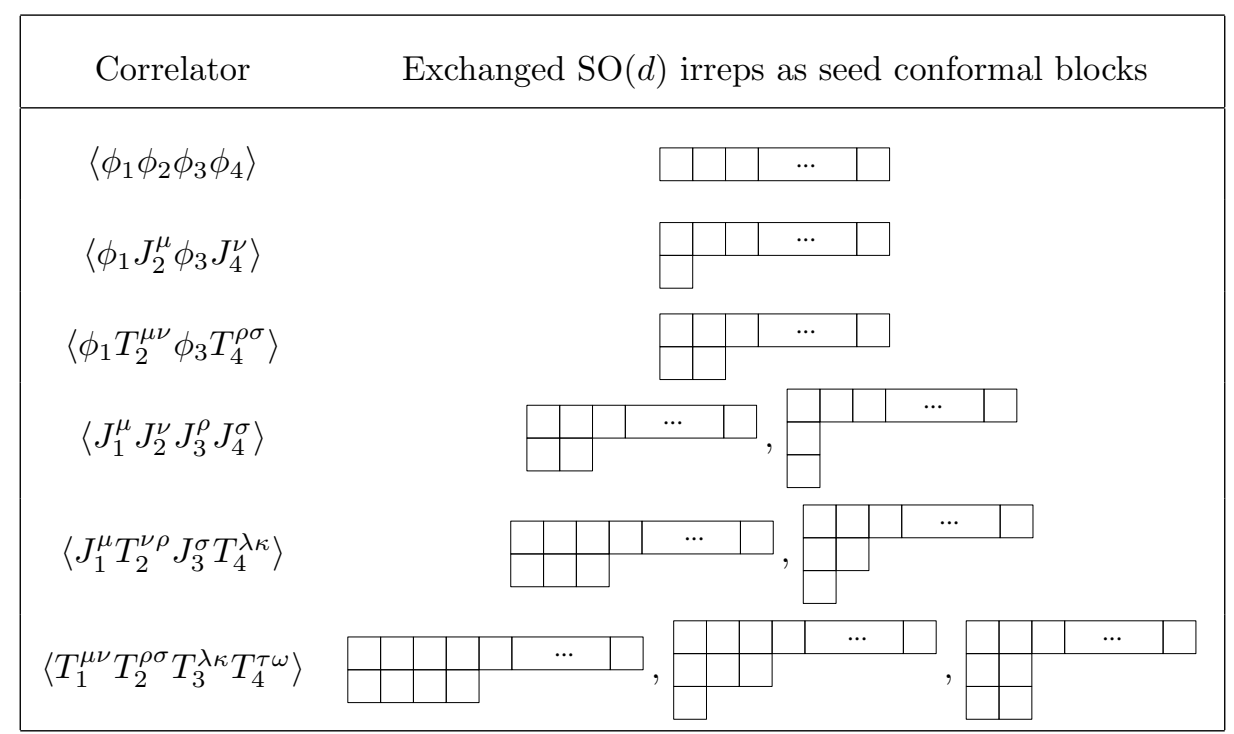

Table 1. Exchanged irreps in correlators of currents and stress-tensors. Each line shows only the irreps exchanged as a seed block. For a correlator in a given line, the irreps in the lines above can also be exchanged, but those conformal blocks can be constructed by acting with derivatives.

\section{Projectors to traceless mixed-symmetry tensors}

\subsection{Review of projectors to traceless symmetric tensors}

As an inspiration for the ideas ahead let us briefly review how to quickly derive the projector to traceless symmetric tensors encoded in a simple polynomial, following [38]. The projector is defined by its symmetry

$$
\pi_{(l)}^{a_{1} \ldots a_{l}, b_{1} \ldots b_{l}}=\pi_{(l)}^{\left(a_{1} \ldots a_{l}\right),\left(b_{1} \ldots b_{l}\right)}
$$

tracelessness

$$
\delta_{a_{1} a_{2}} \pi_{(l)}^{a_{1} \ldots a_{l}, b_{1} \ldots b_{l}}=\pi_{(l)}^{a_{1} \ldots a_{l}, b_{1} \ldots b_{l}} \delta_{b_{1} b_{2}}=0
$$

and idempotence

$$
\pi_{(l)}^{a_{1} \ldots a_{l}, b_{1} \ldots b_{l}} \pi_{(l) b_{1} \ldots b_{l}}{ }^{c_{1} \ldots c_{l}}=\pi_{(l)}^{a_{1} \ldots a_{l}, c_{1} \ldots c_{l}} .
$$

Due to the first property the projector can be implemented as a function of a single variable by contracting with two auxiliary vectors $z^{a}, \bar{z}^{b} \in \mathbb{R}^{d}$,

$$
\pi_{(l)}(z, \bar{z})=z^{a_{1}} \ldots z^{a_{l}} \pi_{(l) a_{1} \ldots a_{l}, b_{1} \ldots b_{l}} \bar{z}^{b_{1}} \ldots \bar{z}^{b_{l}}=\left(z^{2} \bar{z}^{2}\right)^{\frac{l}{2}} f_{l}(t), \quad t=\frac{z \cdot \bar{z}}{\left(z^{2} \bar{z}^{2}\right)^{\frac{1}{2}}}
$$

The tracelessness can be imposed using the differential operator

$$
\frac{\partial}{\partial z} \cdot \frac{\partial}{\partial z} \pi_{\lambda}(z, \bar{z})=0
$$

which results in a Gegenbauer differential equation for $f_{l}(t)$,

$$
\left(t^{2}-1\right) f_{l}^{\prime \prime}(t)+(d-1) t f_{l}^{\prime}(t)=l(l+d-2) f_{l}(t),
$$


constraining $f_{l}(t)$ to be proportional to the Gegenbauer polynomial $C_{l}^{\left(\frac{d}{2}-1\right)}(t)$. The result including normalization is [38]

$$
\pi_{(l)}(z, \bar{z})=c_{(l)}\left(z^{2} \bar{z}^{2}\right)^{\frac{l}{2}} C_{l}^{\left(\frac{d}{2}-1\right)}(t), \quad \text { with } c_{(l)}=\frac{l !}{2^{l}\left(\frac{d}{2}-1\right)_{l}},
$$

where $(x)_{n}=\frac{\Gamma(x+n)}{\Gamma(x)}$ is the Pochhammer symbol.

\subsection{Projectors for Young diagrams with two rows}

Next let us consider the irreps which are labeled by Young diagrams with two rows $\lambda=$ $\left(l_{1}, l_{2}\right)$. The total number of boxes in the Young diagram $\lambda$ will be denoted by $|\lambda|$, so in the case at hand we have $|\lambda|=l_{1}+l_{2}$. Again we want to construct these projectors as polynomials and then impose differential equations. In this case the projector can be encoded in a polynomial depending on four vectors

$$
\pi_{\lambda}\left(z_{1}, z_{2}, \bar{z}_{1}, \bar{z}_{2}\right)=z_{1}^{a_{1}} \ldots z_{1}^{a_{l_{1}}} z_{2}^{a_{l_{1}+1}} \ldots z_{2}^{a_{|\lambda|}} \pi_{\lambda a_{1} \ldots a_{|\lambda|}, b_{1} \ldots b_{|\lambda|}} \bar{z}_{1}^{b_{1}} \ldots \bar{z}_{1}^{b_{l_{1}}} \bar{z}_{2}^{b_{l_{1}+1}} \ldots \bar{z}_{2}^{b_{|\lambda|}} .
$$

Our strategy is now to first implement the mixed-symmetry property and then the tracelessness. Along the way we will always make sure to keep the construction symmetric under exchange of $z_{i}$ and $\bar{z}_{i}$,

$$
\pi_{\lambda}\left(z_{1}, z_{2}, \bar{z}_{1}, \bar{z}_{2}\right)=\pi_{\lambda}\left(\bar{z}_{1}, \bar{z}_{2}, z_{1}, z_{2}\right) .
$$

This means it is enough to impose conditions on one side of the projector.

The mixed symmetry or Young symmetrization of a tensor $f$ in the symmetric representation of the irrep $\left(l_{1}, l_{2}\right)$ amounts to the following two conditions ${ }^{1}$

$$
\begin{aligned}
f_{a_{1} \ldots a_{l_{1}} b_{1} \ldots b_{l_{2}}} & =f_{\left(a_{1} \ldots a_{l_{1}}\right)\left(b_{1} \ldots b_{l_{2}}\right)}, \\
-f_{\left(a_{1} \ldots a_{l_{1}}\right)\left(b_{1} \ldots b_{l_{2}}\right)} & =f_{\left(b_{1} a_{2} \ldots a_{l_{1}}\right)\left(a_{1} b_{2} \ldots b_{l_{2}}\right)}+f_{\left(a_{1} b_{1} a_{3} \ldots a_{l_{1}}\right)\left(a_{2} b_{2} \ldots b_{l_{2}}\right)}+\ldots+f_{\left(a_{1} \ldots a_{l_{1}-1} b_{1}\right)\left(a_{l_{1}} b_{2} \ldots b_{l_{2}}\right)} .
\end{aligned}
$$

The first condition is automatically satisfied after the contraction with polarizations. The second one can be rephrased as

$$
z_{1}^{a_{1}} \ldots z_{1}^{a_{l_{1}}} z_{1}^{b_{1}} z_{2}^{b_{2}} \ldots z_{2}^{b_{l_{2}}} f_{\left(a_{1} \ldots a_{l_{1}}\right)\left(b_{1} \ldots b_{l_{2}}\right)}=0 .
$$

One can see the equivalence by acting on the latter expression with

$$
\partial_{z_{1}^{a_{1}}} \ldots \partial_{z_{1} a_{l_{1}}} \partial_{z_{1}^{b_{1}}} \partial_{z_{2}^{b_{2}}} \ldots \partial_{z_{2}^{b_{l}}}
$$

Hence, the mixed-symmetry property just means that the polynomial must vanish whenever one of the vectors $z_{2}$ is replaced by $z_{1}$, or

$$
\left(z_{1} \cdot \frac{\partial}{\partial z_{2}}\right) \pi_{\lambda}\left(z_{1}, z_{2}, \bar{z}_{1}, \bar{z}_{2}\right)=0 .
$$

\footnotetext{
${ }^{1}$ This is explained in section 2.3 of [45].
} 
This can be easily implemented by constructing $\pi_{\lambda}\left(z_{1}, z_{2}, \bar{z}_{1}, \bar{z}_{2}\right)$ out of structures $Q_{s}$ that have this property

$$
\pi_{\lambda}\left(z_{1}, z_{2}, \bar{z}_{1}, \bar{z}_{2}\right)=c_{\lambda}\left(z_{1}^{2} \bar{z}_{1}^{2}\right)^{\frac{l_{1}}{2}} \sum_{s} f_{s}(t) Q_{s}\left(z_{1}, z_{2}, \bar{z}_{1}, \bar{z}_{2}\right), \quad t=\frac{z_{1} \cdot \bar{z}_{1}}{\left(z_{1}^{2} \bar{z}_{1}^{2}\right)^{\frac{1}{2}}} .
$$

The structures $Q_{s}$ have weight $l_{2}$ in $z_{2}$ and $\bar{z}_{2}$, and zero weight in $z_{1}$ and $\bar{z}_{1}$. We are already familiar with such transverse structures from the construction of conformally invariant correlators in embedding space. These structures can be built out of transverse building blocks. In this case one can use

$$
\begin{gathered}
H\left(z_{2}, \bar{z}_{2}\right)=z_{2}^{a} H_{a b} \bar{z}_{2}^{b}=z_{2}^{a}\left(\delta_{a b}-\frac{\bar{z}_{1 a} z_{1 b}}{z_{1} \cdot \bar{z}_{1}}\right) \bar{z}_{2}^{b}, \\
V\left(z_{2}\right)=z_{2}^{a} V_{a}=z_{2}^{a}\left(\delta_{a b}-\frac{z_{1 a} z_{1 b}}{z_{1}^{2}}\right) \frac{\bar{z}_{1}^{b}}{\sqrt{t \bar{z}_{1}^{2}}}, \\
\bar{V}\left(\bar{z}_{2}\right)=\bar{V}_{b} \bar{z}_{2}^{b}=\frac{z_{1}^{a}}{\sqrt{t z_{1}^{2}}}\left(\delta_{a b}-\frac{\bar{z}_{1 a} \bar{z}_{1 b}}{\bar{z}_{1}^{2}}\right) \bar{z}_{2}^{b}, \\
T\left(z_{2}, z_{2}\right)=z_{2}^{a_{1}} z_{2}^{a_{2}} T_{a_{1} a_{2}}=z_{2}^{a_{1}} z_{2}^{a_{2}}\left(\delta_{a_{1} a_{2}}-\frac{z_{1 a_{1}} z_{1 a_{2}}}{z_{1}^{2}}\right), \\
\bar{T}\left(\bar{z}_{2}, \bar{z}_{2}\right)=\bar{T}_{b_{1} b_{2}} \bar{z}_{2}^{b_{1}} \bar{z}_{2}^{b_{2}}=\left(\delta_{b_{1} b_{2}}-\frac{\bar{z}_{1 b_{1}} \bar{z}_{1 b_{2}}}{\bar{z}_{1}^{2}}\right) \bar{z}_{2}^{b_{1}} \bar{z}_{2}^{b_{2}} .
\end{gathered}
$$

This construction ensures that the number of undetermined functions $f_{s}(t)$ is as small as possible. Note that the dependence of the building blocks on $t$ is

$$
\begin{aligned}
H\left(z_{2}, \bar{z}_{2}\right) & =\mathcal{O}(1)+\mathcal{O}\left(t^{-1}\right), \\
V\left(z_{2}\right), \bar{V}\left(\bar{z}_{2}\right) & =\mathcal{O}\left(t^{-\frac{1}{2}}\right)+\mathcal{O}\left(t^{\frac{1}{2}}\right), \\
T\left(z_{2}, z_{2}\right), \bar{T}\left(\bar{z}_{2}, \bar{z}_{2}\right) & =\mathcal{O}(1) .
\end{aligned}
$$

The individual terms in the sum of (2.14) can have negative powers of $t$, however any such terms must cancel in the sum. The building blocks were chosen such that the $f_{s}(t)$ are polynomials of minimal degree, i.e. they do not have overall factors of $t$. This can be demonstrated by considering the example $\lambda=\left(l_{1}, 1\right)$, and using that $\pi_{\lambda}\left(z_{1}, z_{2}, \bar{z}_{1}, \bar{z}_{2}\right)$ must be a polynomial in products of $z_{1}, z_{2}, \bar{z}_{1}, \bar{z}_{2}$, that is

$$
\begin{aligned}
\pi_{\left(l_{1}, 1\right)} & \left(z_{1}, z_{2}, \bar{z}_{1}, \bar{z}_{2}\right) \\
\propto & \left(z_{1}^{2} \bar{z}_{1}^{2}\right)^{\frac{l_{1}}{2}}\left(f_{1}(t) H\left(z_{2}, \bar{z}_{2}\right)+f_{2}(t) V\left(z_{2}\right) \bar{V}\left(\bar{z}_{2}\right)\right) \\
= & \left(z_{1}^{2} \bar{z}_{1}^{2}\right)^{\frac{l_{1}}{2}}\left(z_{2} \cdot \bar{z}_{2}\right) f_{1}(t) \\
& \quad-\left(z_{1}^{2} \bar{z}_{1}^{2}\right)^{\frac{l_{1}-1}{2}}\left(z_{1} \cdot \bar{z}_{2}\right)\left(z_{2} \cdot \bar{z}_{1}\right)\left(\frac{f_{1}(t)-f_{2}(t)}{t}\right) \\
& \quad+\left(z_{1}^{2} \bar{z}_{1}^{2}\right)^{\frac{l_{1}-2}{2}}\left(\left(z_{1} \cdot z_{2}\right)\left(\bar{z}_{1} \cdot \bar{z}_{2}\right)\left(z_{1} \cdot \bar{z}_{1}\right)-\left(z_{1} \cdot z_{2}\right)\left(z_{1} \cdot \bar{z}_{2}\right) \bar{z}_{1}^{2}-\left(\bar{z}_{1} \cdot z_{2}\right)\left(\bar{z}_{1} \cdot \bar{z}_{2}\right) z_{1}^{2}\right) f_{2}(t) .
\end{aligned}
$$

From the second, third and fourth line of this equation we conclude that

$$
f_{1}(t)=\sum_{i=0}^{l_{1}} a_{i} t^{i}, \quad \frac{f_{1}(t)-f_{2}(t)}{t}=\sum_{i=0}^{l_{1}-1} b_{i} t^{i}, \quad f_{2}(t)=\sum_{i=0}^{l_{1}-2} c_{i} t^{i},
$$


respectively. Combining these three conditions yields for this case

$$
f_{1}(t)=\sum_{i=0}^{l_{1}} a_{i} t^{i}, \quad f_{2}(t)=\sum_{i=0}^{l_{1}-2} c_{i} t^{i}, \quad a_{0}=c_{0} .
$$

Furthermore, both functions have to be even to avoid a square root in $z_{1}^{2} \bar{z}_{1}^{2}$.

These polynomials are determined by solving a coupled system of second order differential equations arising from the tracelessness conditions

$$
\frac{\partial}{\partial z_{1}} \cdot \frac{\partial}{\partial z_{1}} \pi_{\lambda}\left(z_{1}, z_{2}, \bar{z}_{1}, \bar{z}_{2}\right)=\frac{\partial}{\partial z_{1}} \cdot \frac{\partial}{\partial z_{2}} \pi_{\lambda}\left(z_{1}, z_{2}, \bar{z}_{1}, \bar{z}_{2}\right)=\frac{\partial}{\partial z_{2}} \cdot \frac{\partial}{\partial z_{2}} \pi_{\lambda}\left(z_{1}, z_{2}, \bar{z}_{1}, \bar{z}_{2}\right)=0 .
$$

After discussing a first example, we will describe the algorithm that is used to solve these equations in section 2.2.3. Then the structures of the individual families of projectors will be presented. Finally, the overall normalization constants $c_{\lambda}$ appearing in (2.14) will be computed in section 2.4 .

\subsubsection{Birdtrack notation}

To construct Young symmetrized structures $Q_{i}$ it is convenient to use birdtrack notation, with lines denoting index contractions. Using multiple copies of the same vector results in a group of symmetric indices, which is denoted by a white bar

$$
z \longleftarrow a_{a_{2}}^{a_{1}}=z_{a_{1}} z_{a_{2}}
$$

while a black bar denotes antisymmetrization

$$
a_{a_{2}}^{a_{1}} \boldsymbol{Z}_{b_{2}}^{b_{1}}=\frac{1}{2}\left(\delta_{a_{1} b_{1}} \delta_{a_{2} b_{2}}-\delta_{a_{1} b_{2}} \delta_{a_{2} b_{1}}\right) .
$$

The building blocks transverse to $z_{1}^{a}$ and to $\bar{z}_{1}^{b}$ that were defined in (2.15) will be denoted by the following symbols

$$
\begin{aligned}
H_{a b} & =a \multimap b, & \delta_{a b} & =a-b, \\
V_{a} & =a \longrightarrow, & \bar{V}_{b} & =\curvearrowleft b, \\
T_{a_{1} a_{2}} & =a_{a_{2}} a_{1} \supset, & \bar{T}_{b_{1} b_{2}} & =\complement_{b_{2}}^{b_{1}} .
\end{aligned}
$$

The $\delta_{a b}$ was defined to indicate that short lines connecting $\left[-\right.$ and $\mathbf{z}=$ do not stand for $H_{a b}$. The notation should be clear after the first examples which are given both in birdtrack and explicit notation.

\subsubsection{Projectors to the irreps $\left(l_{1}, 1\right)$}

The projectors to $\mathrm{SO}(d)$ irreps with Young diagrams of shape $\square^{11 \cdots} \square$ were already derived in [42]. We include them here for completeness. The structures are

$$
\begin{aligned}
& Q_{1}=z_{2} \square \square \square \bar{z}_{2}=H\left(z_{2}, \bar{z}_{2}\right), \\
& Q_{2}=z_{2} \square \bullet \bullet \square \bar{z}_{2}=V\left(z_{2}\right) \bar{V}\left(\bar{z}_{2}\right) .
\end{aligned}
$$


Imposing the tracelessness conditions (2.20) results in many differential equations for the functions $f_{1}(t)$ and $f_{2}(t)$. For example the term proportional to $\left(z_{1} \cdot z_{2}\right)\left(z_{1} \cdot \bar{z}_{2}\right)$ in the condition $\frac{\partial}{\partial z_{1}} \cdot \frac{\partial}{\partial z_{1}} \pi_{\lambda}\left(z_{1}, z_{2}, \bar{z}_{1}, \bar{z}_{2}\right)=0$ is

$$
-\left(l_{1}-2\right)\left(l_{1}+d\right) f_{2}(t)+(d+3) t f_{2}^{\prime}(t)+\left(t^{2}-1\right) f_{2}^{\prime \prime}(t)=0 .
$$

This is the Gegenbauer differential equation, solved by $f_{2}(t)=-\partial_{t}^{2} C_{l_{1}}^{\left(\frac{d}{2}-1\right)}(t) \propto C_{l_{1}-2}^{\left(\frac{d}{2}+1\right)}(t)$. The full solution is

$$
\begin{aligned}
& f_{1}(t)=(d-2) t \partial_{t} C_{l_{1}}^{\left(\frac{d}{2}-1\right)}(t)+\left(t^{2}-1\right) \partial_{t}^{2} C_{l_{1}}^{\left(\frac{d}{2}-1\right)}(t), \\
& f_{2}(t)=-\partial_{t}^{2} C_{l_{1}}^{\left(\frac{d}{2}-1\right)}(t) .
\end{aligned}
$$

In the next section we describe the algorithm that was used to automatize the process of solving the differential equations in all other cases.

\subsubsection{Algorithm for solving the differential equations}

The algorithm for solving the systems of differential equations we encounter is based on the assumption that all polynomials $f_{s}(t)$ can be written as a finite sum of derivatives of the Gegenbauer polynomial $C_{l_{1}}^{\left(\frac{d}{2}-1\right)}(t)$, which will be denoted by

$$
\mathcal{C}_{l_{1}}^{(n)}(t) \equiv \partial_{t}^{n} C_{l_{1}}^{\left(\frac{d}{2}-1\right)}(t)=2^{n}\left(\frac{d}{2}-1\right)_{n} C_{l_{1}-n}^{\left(\frac{d}{2}-1+n\right)}(t)
$$

with $l_{1}$-independent coefficients $w_{s, n}(d, t)$. That is, we write

$$
f_{s}(t)=\sum_{n} w_{s, n}(d, t) \mathcal{C}_{l_{1}}^{(n)}(t)
$$

This assumption turns out to be true for all computed projectors, with the sum ranging from $n=l_{2}$ up to $n=2 l_{2}$. The overall normalization of all functions $f_{s}(t)$ in a projector will be chosen such that ${ }^{2}$

$$
w_{1,2 l_{2}}(d, t)=t^{2 l_{2}}+\mathcal{O}\left(t^{2 l_{2}-2}\right) .
$$

The independence on $l_{1}$ of the coefficients is relevant for the derivation of recursion relations for conformal blocks.

We will use the following relation, which is a version of the Gegenbauer differential equation for the Gegenbauer polynomial appearing explicitly in (2.27),

$$
\left(l_{1}-n\right)\left(l_{1}+n+d-2\right) \mathcal{C}_{l_{1}}^{(n)}(t)=(d+2 n-1) t \mathcal{C}_{l_{1}}^{(n+1)}(t)+\left(t^{2}-1\right) \mathcal{C}_{l_{1}}^{(n+2)}(t) .
$$

Using this relation repeatedly on a polynomial of the form (2.28) of order $l_{1}$ or lower, one can remove all but the two highest derivatives and write it in the following way

$$
f_{s}(t)=\sum_{i=0}^{N} u_{s, i}\left(d, l_{1}\right) t^{i} \mathcal{C}_{l_{1}}^{(N)}(t)+\sum_{j=0}^{N-1} v_{s, j}\left(d, l_{1}\right) t^{j} \mathcal{C}_{l_{1}}^{(N-1)}(t),
$$

\footnotetext{
${ }^{2}$ The functions $f_{1}(t)$ are special in that the corresponding $Q_{1}$ are chosen to contain only $H$ building blocks.
} 
where $N$ is the highest $n$ appearing in the sum in (2.28). Plugging this ansatz into the differential equations that arise from demanding tracelessness results in a system of linear equations. In all cases discussed below, these linear systems have a unique nontrivial solution for $u_{s, i}\left(d, l_{1}\right)$ and $v_{s, j}\left(d, l_{1}\right)$, as long as one chooses $N$ large enough. After finding the solution, $(2.30)$ can again be used to bring the solution into a form with $l_{1}$-independent coefficients.

\subsubsection{Projectors to the irreps $\left(l_{1}, 2\right)$}

One can easily convince oneself that for Young diagrams of shape $\Psi^{\prime \cdots} \square$ the possible combinations of the proposed building blocks are

$$
\begin{aligned}
& Q_{1}=z_{2} \square \square \bar{z}_{2}=H\left(z_{2}, \bar{z}_{2}\right)^{2}, \\
& Q_{2}=z_{2} \bigsqcup \bullet \square \bar{z}_{2}=H\left(z_{2}, \bar{z}_{2}\right) V\left(z_{2}\right) \bar{V}\left(\bar{z}_{2}\right) \text {, } \\
& Q_{3}=z_{2} \square \rightarrow-\square \bar{z}_{2}=V\left(z_{2}\right)^{2} \bar{V}\left(\bar{z}_{2}\right)^{2} \text {, } \\
& Q_{4}=z_{2} \longmapsto \subset \bar{z}_{2}=T\left(z_{2}, z_{2}\right) \bar{T}\left(\bar{z}_{2}, \bar{z}_{2}\right) \text {, } \\
& Q_{5}=z_{2} \bigsqcup \bullet \bar{z}_{2}+z_{2} \bigsqcup ّ \bar{z}_{2}=T\left(z_{2}, z_{2}\right) \bar{V}\left(\bar{z}_{2}\right)^{2}+V\left(z_{2}\right)^{2} \bar{T}\left(\bar{z}_{2}, \bar{z}_{2}\right) \text {. }
\end{aligned}
$$

Using the algorithm for solving the tracelessness conditions one finds that the functions $f_{i}(t)$ can be expressed $a^{3}$

$$
f_{i}(t)=-\frac{\hat{f}_{i}(t)}{d-2}, \quad \forall i=1, \ldots, 5
$$

with

$$
\begin{aligned}
& \hat{f}_{1}(t)=(d-1) d\left(1-(d-2) t^{2}\right) \mathcal{C}_{l_{1}}^{(2)}(t)-2(d-2) d t\left(t^{2}-1\right) \mathcal{C}_{l_{1}}^{(3)}(t)-(d-2)\left(t^{2}-1\right)^{2} \mathcal{C}_{l_{1}}^{(4)}(t), \\
& \hat{f}_{2}(t)=2(d-1) d \mathcal{C}_{l_{1}}^{(2)}(t)+2(d-1) d t \mathcal{C}_{l_{1}}^{(3)}(t)+2(d-2)\left(t^{2}-1\right) \mathcal{C}_{l_{1}}^{(4)}(t), \\
& \hat{f}_{3}(t)=(d-1) d \mathcal{C}_{l_{1}}^{(2)}(t)+2 d t \mathcal{C}_{l_{1}}^{(3)}(t)+\left(2-d+t^{2}\right) \mathcal{C}_{l_{1}}^{(4)}(t), \\
& \hat{f}_{4}(t)=d\left(-2+(d-1) t^{2}\right) \mathcal{C}_{l_{1}}^{(2)}(t)+2 d t\left(t^{2}-1\right) \mathcal{C}_{l_{1}}^{(3)}(t)+\left(t^{2}-1\right)^{2} \mathcal{C}_{l_{1}}^{(4)}(t), \\
& \hat{f}_{5}(t)=(d-1) d t \mathcal{C}_{l_{1}}^{(2)}(t)+2 d t^{2} \mathcal{C}_{l_{1}}^{(3)}(t)+t\left(t^{2}-1\right) \mathcal{C}_{l_{1}}^{(4)}(t) .
\end{aligned}
$$

The projectors to the families of irreps $\left(l_{1}, 3\right)$ and $\left(l_{1}, 4\right)$ are given in appendix $\mathrm{C}$.

\subsection{Projectors for Young diagrams with three rows}

Next projectors to tensors corresponding to Young diagrams with three rows will be discussed. Such tensors can be encoded with three auxiliary vectors $z_{i}(i=1,2,3)$, hence for the projector we will consider

$$
\begin{aligned}
& \pi_{\lambda}\left(\left\{z_{i}, \bar{z}_{i}\right\}\right) \\
& \quad \equiv \pi_{\lambda}\left(z_{1}, z_{2}, z_{3}, \bar{z}_{1}, \bar{z}_{2}, \bar{z}_{3}\right) \\
& \quad=z_{1}^{a_{1}} \ldots z_{1}^{a_{1}} z_{2}^{a_{l_{1}+1}} \ldots z_{2}^{a_{l_{1}+l_{2}}} z_{3}^{a_{l_{1}+l_{2}+1} \ldots z_{3}^{a_{|\lambda|}}} \pi_{\lambda a_{1} \ldots a_{|\lambda|}, b_{1} \ldots b_{|\lambda|}} \bar{z}_{1}^{b_{1}} \ldots \bar{z}_{1}^{b_{1}} \bar{z}_{2}^{b_{l_{1}+1} \ldots} \bar{z}_{2}^{b_{1}+l_{2}} \bar{z}_{3}^{a_{l_{1}+l_{2}+1}} \ldots \bar{z}_{3}^{a_{|\lambda|}} .
\end{aligned}
$$

\footnotetext{
${ }^{3}$ Note that the overall denominator introduced in the first line is required for our normalization (2.29).
} 
The mixed-symmetry property amounts to relations such as (2.10), but now such relations hold between any two of the three sets of symmetrized indices. With the same argument as in the case of two-row Young diagrams, this property can be imposed on $\pi_{\lambda}$ by requiring

$$
\left(z_{1} \cdot \frac{\partial}{\partial z_{2}}\right) \pi_{\lambda}\left(\left\{z_{i}, \bar{z}_{i}\right\}\right)=\left(z_{1} \cdot \frac{\partial}{\partial z_{3}}\right) \pi_{\lambda}\left(\left\{z_{i}, \bar{z}_{i}\right\}\right)=\left(z_{2} \cdot \frac{\partial}{\partial z_{3}}\right) \pi_{\lambda}\left(\left\{z_{i}, \bar{z}_{i}\right\}\right)=0 .
$$

The first two conditions can again be implemented by using the building blocks defined in (2.15), now also allowing $z_{3}, \bar{z}_{3}$ in the place of $z_{2}, \bar{z}_{2}$. The third condition can be implemented by Young symmetrizing in $z_{2}$ and $z_{3}$ (and $\bar{z}_{2}, \bar{z}_{3}$ ). As before, this leads to the following ansatz separating structures depending on $z_{2}, z_{3}, \bar{z}_{2}, \bar{z}_{3}$ and functions of $t$

$$
\pi_{\lambda}\left(\left\{z_{i}, \bar{z}_{i}\right\}\right)=c_{\lambda}\left(z_{1}^{2} \bar{z}_{1}^{2}\right)^{\frac{l_{1}}{2}} \sum_{s} f_{s}(t) Q_{s}\left(z_{1}, z_{2}, z_{3}, \bar{z}_{1}, \bar{z}_{2}, \bar{z}_{3}\right)
$$

Tracelessness now amounts to the old equations (2.20) and to the three new ones

$$
\frac{\partial}{\partial z_{1}} \cdot \frac{\partial}{\partial z_{3}} \pi_{\lambda}\left(\left\{z_{i}, \bar{z}_{i}\right\}\right)=\frac{\partial}{\partial z_{2}} \cdot \frac{\partial}{\partial z_{3}} \pi_{\lambda}\left(\left\{z_{i}, \bar{z}_{i}\right\}\right)=\frac{\partial}{\partial z_{3}} \cdot \frac{\partial}{\partial z_{3}} \pi_{\lambda}\left(\left\{z_{i}, \bar{z}_{i}\right\}\right)=0 .
$$

\subsubsection{Projectors to the irreps $\left(l_{1}, 1,1\right)$}

For Young diagrams of shape antisymmetry between $z_{2}, z_{3}$ and $\bar{z}_{2}, \bar{z}_{3}$,

$$
\begin{aligned}
& Q_{1}=z_{z_{3}} \text { 口—目 } \bar{z}_{2}=\frac{1}{2}\left(H\left(z_{2}, \bar{z}_{2}\right) H\left(z_{3}, \bar{z}_{3}\right)-H\left(z_{2}, \bar{z}_{3}\right) H\left(z_{3}, \bar{z}_{2}\right)\right) \text {, }
\end{aligned}
$$

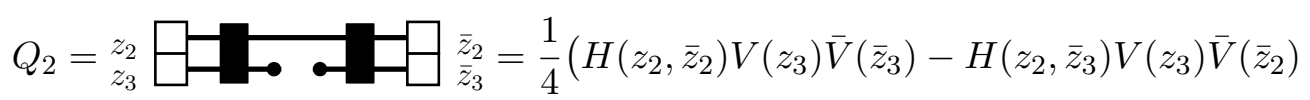

$$
\begin{aligned}
& \left.-H\left(z_{3}, \bar{z}_{2}\right) V\left(z_{2}\right) \bar{V}\left(\bar{z}_{3}\right)+H\left(z_{3}, \bar{z}_{3}\right) V\left(z_{2}\right) \bar{V}\left(\bar{z}_{2}\right)\right) \text {. }
\end{aligned}
$$

The resulting functions $f_{i}(t)$ are similar as in the case of irreps $\square+\cdots$ in (2.26),

$$
\begin{aligned}
& f_{1}(t)=(d-3) t \mathcal{C}_{l_{1}}^{(1)}(t)+\left(t^{2}-1\right) \mathcal{C}_{l_{1}}^{(2)}(t), \\
& f_{2}(t)=-2 \mathcal{C}_{l_{1}}^{(2)}(t) .
\end{aligned}
$$

\subsubsection{Projectors to the irreps $\left(l_{1}, 2,1\right)$}

Starting with this example, whose Young diagrams have shape $\bigoplus^{\cdots} \square$, it becomes helpful to consider some tensor products to make sure that one finds the correct number of structures. As explained above, the allowed structures are constructed from the building blocks in (2.15), including also the dependence on $z_{3}, \bar{z}_{3}$ in the place of $z_{2}, \bar{z}_{2}$. In order to satisfy the condition $\left(z_{2} \cdot \frac{\partial}{\partial z_{3}}\right) Q_{i}=0$ of $(2.36)$ it is enough to Young symmetrize in $z_{2}$ and $z_{3}$ according to the Young diagram $\left(l_{2}, l_{3}\right)=(2,1)$. In the case at hand that means to consider

$$
z_{2}={ }_{z_{3}}^{a_{2}}=z_{2}^{a_{1}} z_{2}^{b_{1}} z_{3}^{b_{2}} \frac{1}{2}\left(\delta^{b_{1} a_{2}} \delta^{b_{2} a_{3}}-\delta^{b_{1} a_{3}} \delta^{b_{2} a_{2}}\right) .
$$

Although this expression is a mixed-symmetry tensor, it is not traceless, hence it is in the reducible representation

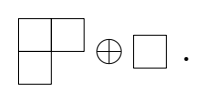


The only way to contract the building block $T_{a_{1} a_{2}}$ to expression (2.41) is

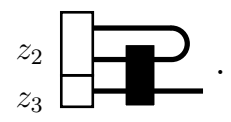

This term contains a contraction with the primitive $\mathrm{SO}(d)$ invariant $\delta_{a_{1} a_{2}}$. Thus, the number of possible contractions with $T_{a_{1} a_{2}}$ is the same as with $\delta_{a_{1} a_{2}}$. We conclude that the number of independent contractions of (2.41) to the corresponding expression with $\bar{z}_{2}$, $\bar{z}_{3}$, with $H$ and $T$ building blocks in the middle, is given by the multiplicity of the scalar representation $\bullet$ in the $\mathrm{SO}(d)$ tensor product ${ }^{4}$

$$
(\square \oplus \square) \otimes(\square \oplus \square)
$$

Since one can also use multiple copies of the building blocks $V$ and $\bar{V}$, which form a symmetric representation, one can then take a further tensor product with a one-row Young diagram of any length. Thus, the total number of birdtracks that one has to consider is the multiplicity of the scalar representation in the tensor product

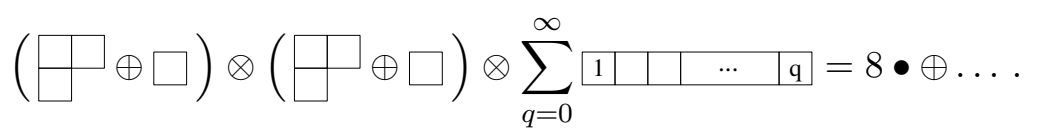

Two of these eight birdtracks are combined into a single structure $\left(Q_{7}\right.$ below), when requiring the building blocks to respect the left-right symmetry $z_{i} \leftrightarrow \bar{z}_{i}$. The resulting structures are

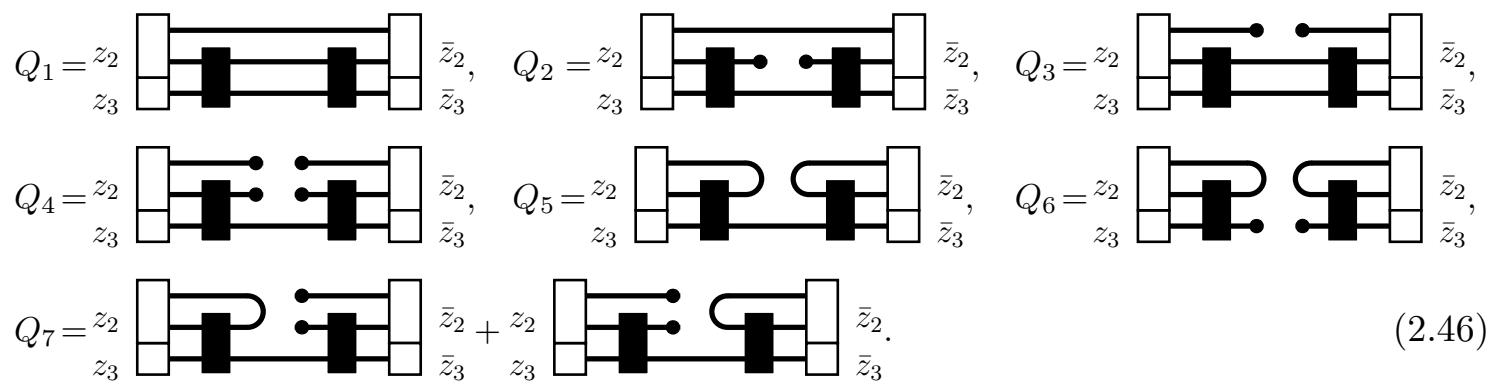

It can be helpful to make a correspondence between the irreps in the tensor product (2.45) and the expressions (2.41) and (2.43)
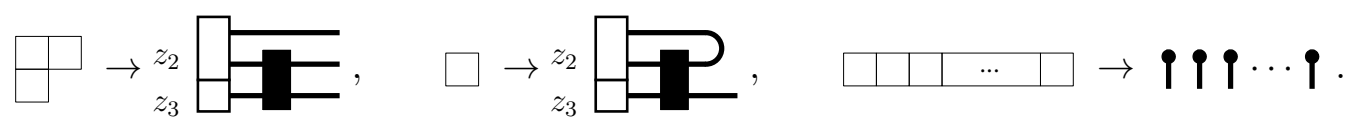

This is not a precise matching, for instance (2.41) is not traceless, however it can be helpful to find a set of independent structures such as (2.46). Using this correspondence one can map the structures to individual contributions in the tensor product (2.45) as follows

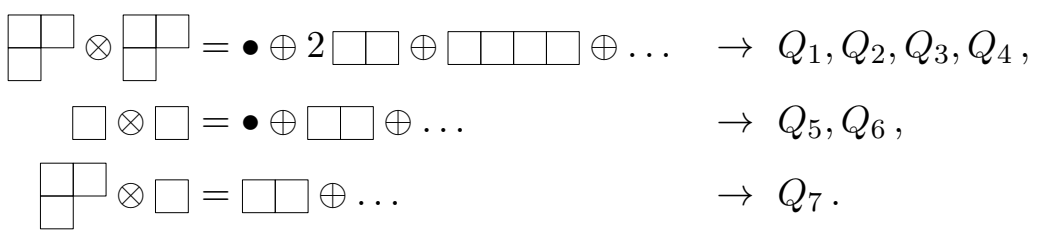

\footnotetext{
${ }^{4} \mathrm{All} \mathrm{SO}(d)$ tensor products in this work are done assuming that $d$ is sufficiently large to make the tensor product $d$ independent, see [45].
} 
The dots here indicate Young diagrams with more than one row, which cannot contribute to the multiplicity of the scalar representation in (2.45). The solution of the tracelessness conditions can then be written as

$$
f_{i}(t)=-\frac{\hat{f}_{i}(t)}{d-3}, \quad \forall i=1, \ldots, 7,
$$

with

$$
\begin{aligned}
\hat{f}_{1}(t)= & -(d-2) d\left(-1+(d-3) t^{2}\right) \mathcal{C}_{l_{1}}^{(2)}(t)-(d-3)(2 d-1) t\left(t^{2}-1\right) \mathcal{C}_{l_{1}}^{(3)}(t) \\
& -(d-3)\left(t^{2}-1\right)^{2} \mathcal{C}_{l_{1}}^{(4)}(t) \\
\hat{f}_{2}(t)= & 2(d-2) d \mathcal{C}_{l_{1}}^{(2)}(t)+2(-5+(d-1) d) t \mathcal{C}_{l_{1}}^{(3)}(t)+2(d-3)\left(t^{2}-1\right) \mathcal{C}_{l_{1}}^{(4)}(t), \\
\hat{f}_{3}(t)= & (d-2) d \mathcal{C}_{l_{1}}^{(2)}(t)+(7+(d-4) d) t \mathcal{C}_{l_{1}}^{(3)}(t)+(d-3)\left(t^{2}-1\right) \mathcal{C}_{l_{1}}^{(4)}(t) \\
\hat{f}_{4}(t)= & 2(d-2) d \mathcal{C}_{l_{1}}^{(2)}(t)+2(2 d-1) t \mathcal{C}_{l_{1}}^{(3)}(t)+2\left(3-d+t^{2}\right) \mathcal{C}_{l_{1}}^{(4)}(t) \\
\hat{f}_{5}(t)= & 2 d\left(-2+(d-2) t^{2}\right) \mathcal{C}_{l_{1}}^{(2)}(t)+2(2 d-1) t\left(t^{2}-1\right) \mathcal{C}_{l_{1}}^{(3)}(t)+2\left(t^{2}-1\right)^{2} \mathcal{C}_{l_{1}}^{(4)}(t), \\
\hat{f}_{6}(t)= & -4 d \mathcal{C}_{l_{1}}^{(2)}(t)-2(3+d) t \mathcal{C}_{l_{1}}^{(3)}(t)+\left(2-2 t^{2}\right) \mathcal{C}_{l_{1}}^{(4)}(t), \\
\hat{f}_{7}(t)= & 2(d-2) d t \mathcal{C}_{l_{1}}^{(2)}(t)+2(2 d-1) t^{2} \mathcal{C}_{l_{1}}^{(3)}(t)+2 t\left(t^{2}-1\right) \mathcal{C}_{l_{1}}^{(4)}(t)
\end{aligned}
$$

\subsubsection{Projectors to the irreps $\left(l_{1}, 2,2\right)$}

The tensor structures for projectors to irreps $\bigoplus^{1 \cdots}$ are constructed with the reducible representation for the shape $\boxplus$, given by

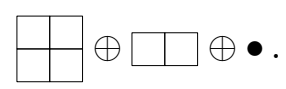

Possible contractions with $T_{a_{1} a_{2}}$ are
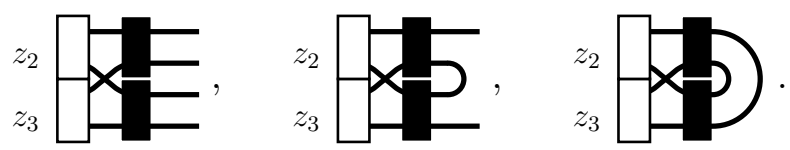

Hence the number of birdtracks to consider is given by

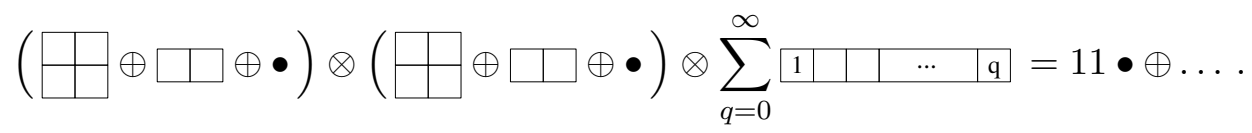

The individual contributions are

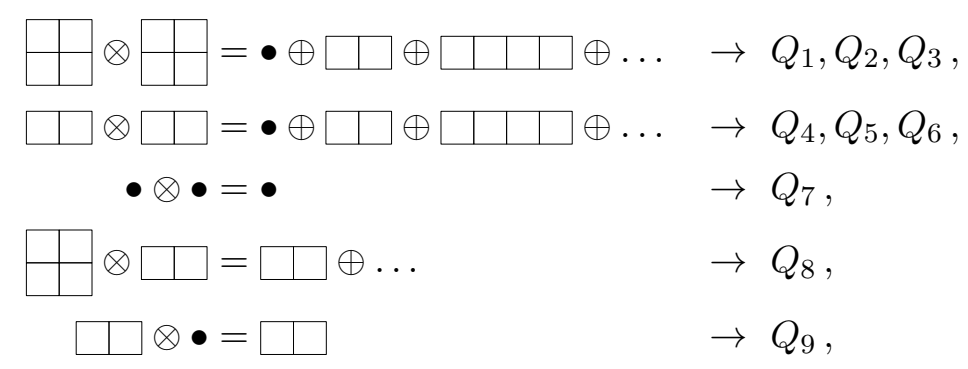


with

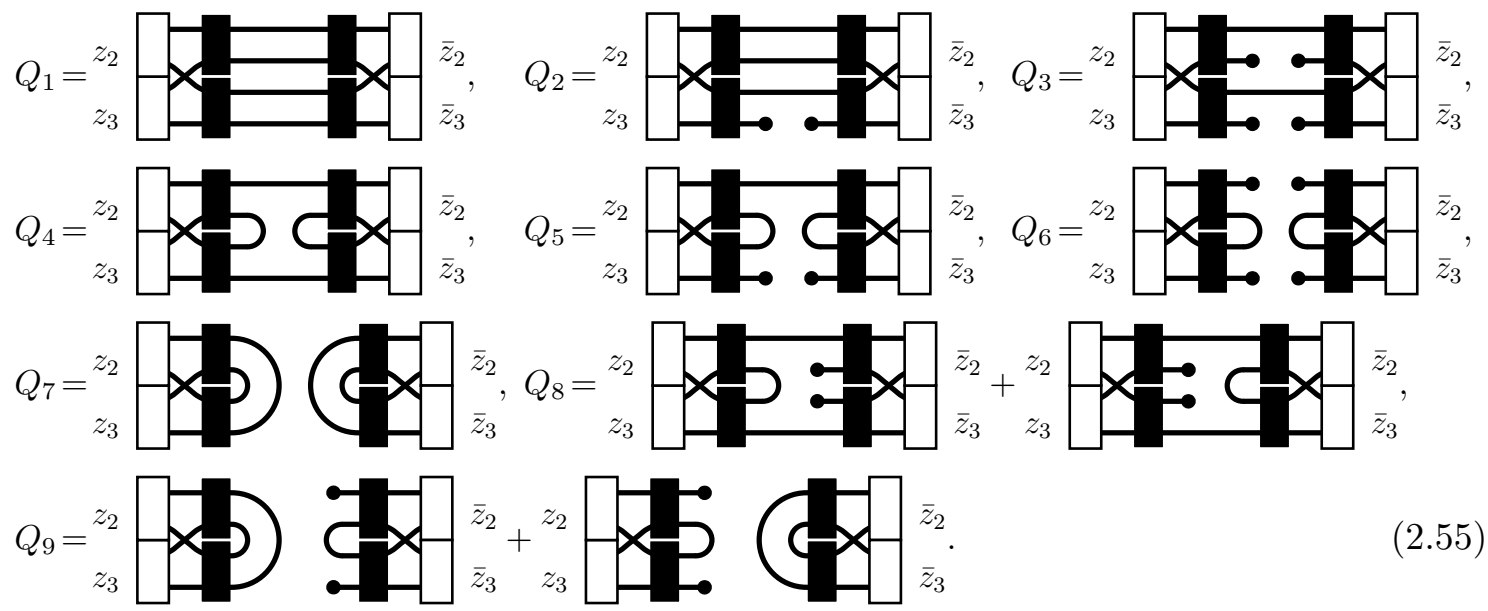

In this case the functions $f_{i}(t)$ can be written as

$$
f_{i}(t)=-\frac{\hat{f}_{i}(t)}{(d-4)(d-3)}, \quad \forall i=1, \ldots, 9,
$$

with

$$
\begin{aligned}
\hat{f}_{1}(t)= & -(d-3)_{3}\left(-1+(d-4) t^{2}\right) \mathcal{C}_{l_{1}}^{(2)}(t)-2(d-4)_{2}(d-1) t\left(t^{2}-1\right) \mathcal{C}_{l_{1}}^{(3)}(t) \\
& -(d-4)_{2}\left(t^{2}-1\right)^{2} \mathcal{C}_{l_{1}}^{(4)}(t) \\
\hat{f}_{2}(t)= & 4(d-3)_{3} \mathcal{C}_{l_{1}}^{(2)}(t)+4(d-3)^{2}(d-1) t \mathcal{C}_{l_{1}}^{(3)}(t)+4(d-4)_{2}\left(t^{2}-1\right) \mathcal{C}_{l_{1}}^{(4)}(t), \\
\hat{f}_{3}(t)= & 4(d-3)_{3} \mathcal{C}_{l_{1}}^{(2)}(t)+8(d-3)(d-1) t \mathcal{C}_{l_{1}}^{(3)}(t)+4(d-3)\left(4-d+t^{2}\right) \mathcal{C}_{l_{1}}^{(4)}(t), \\
\hat{f}_{4}(t)= & 4(d-2)(d-1)\left(-2+(d-3) t^{2}\right) \mathcal{C}_{l_{1}}^{(2)}(t)+8(d-3)(d-1) t\left(t^{2}-1\right) \mathcal{C}_{l_{1}}^{(3)}(t) \\
& +4(d-3)\left(t^{2}-1\right)^{2} \mathcal{C}_{l_{1}}^{(4)}(t), \\
\hat{f}_{5}(t)= & -16(d-2)(d-1) \mathcal{C}_{l_{1}}^{(2)}(t)-8(d-1)^{2} t \mathcal{C}_{l_{1}}^{(3)}(t)-8(d-3)\left(t^{2}-1\right) \mathcal{C}_{l_{1}}^{(4)}(t), \\
\hat{f}_{6}(t)= & -8(d-2)(d-1) \mathcal{C}_{l_{1}}^{(2)}(t)-16(d-1) t \mathcal{C}_{l_{1}}^{(3)}(t)+4\left(-3+d-2 t^{2}\right) \mathcal{C}_{l_{1}}^{(4)}(t), \\
\hat{f}_{7}(t)= & -2(d-1)\left(-3+(d-2) t^{2}\right) \mathcal{C}_{l_{1}}^{(2)}(t)-4(d-1) t\left(t^{2}-1\right) \mathcal{C}_{l_{1}}^{(3)}(t)-2\left(t^{2}-1\right)^{2} \mathcal{C}_{l_{1}}^{(4)}(t), \\
\hat{f}_{8}(t)= & 4(d-3){ }_{3} t \mathcal{C}_{l_{1}}^{(2)}(t)+8(d-3)(d-1) t^{2} \mathcal{C}_{l_{1}}^{(3)}(t)+4(d-3) t\left(t^{2}-1\right) \mathcal{C}_{l_{1}}^{(4)}(t), \\
\hat{f}_{9}(t)= & -4(d-2)(d-1) t \mathcal{C}_{l_{1}}^{(2)}(t)-8(d-1) t^{2} \mathcal{C}_{l_{1}}^{(3)}(t)-4 t\left(t^{2}-1\right) \mathcal{C}_{l_{1}}^{(4)}(t)
\end{aligned}
$$

The projector to the family of irreps $\left(l_{1}, 3,1\right)$ is given in appendix $\mathrm{C}$.

\subsection{Normalization of the projectors}

The normalizations can be computed by using that the projectors have a term with a known normalization, namely the term projecting to generic mixed-symmetry tensors, from which the traces are subtracted by further terms. This is the only term in $\pi_{\lambda}\left(z_{1}, z_{2}, z_{3}, \bar{z}_{1}, \bar{z}_{2}, \bar{z}_{3}\right)$ containing a factor $\left(z_{1} \cdot \bar{z}_{1}\right)^{l_{1}}\left(z_{2} \cdot \bar{z}_{2}\right)^{l_{2}}\left(z_{3} \cdot \bar{z}_{3}\right)^{l_{3}}$. For concreteness let us consider the Young 
diagram $\lambda=\bigoplus^{1 \cdots}$. In this section all lines in birdtracks denote just simple contractions, without any of the definitions of (2.23)

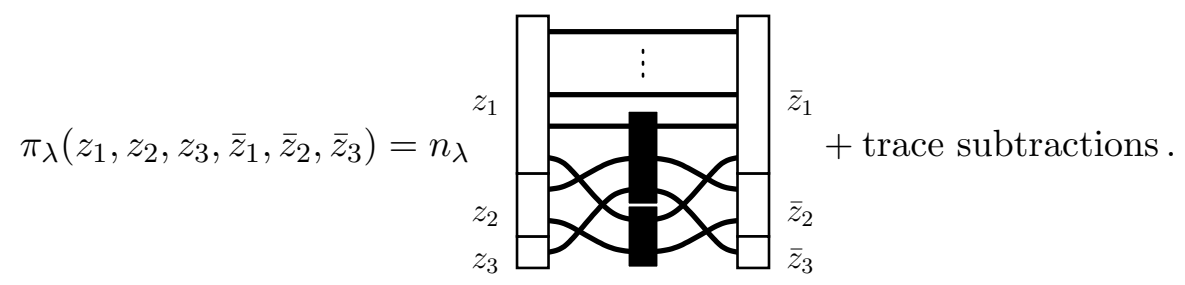

The antisymmetrizations appearing here are defined as

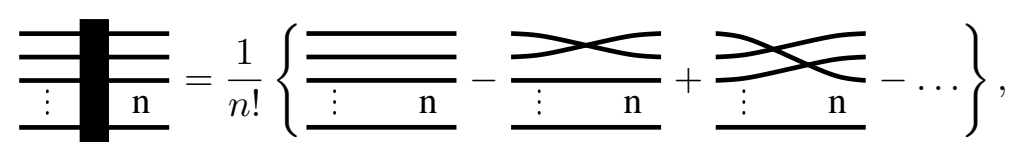

and the normalization of the first term is [46]

$$
n_{\lambda}=\frac{\prod_{i=1}^{l_{1}} h_{i} ! \prod_{j=1}^{h_{1}} l_{j} !}{H(\lambda)}, \quad H(\lambda)=\prod_{i=1}^{l_{1}} \prod_{j=1}^{h_{i}}\left(l_{j}-i+h_{i}-j+1\right),
$$

where $h_{i}$ is the height of the $i^{\text {th }}$ column of the Young diagram $\lambda$. Using the identity

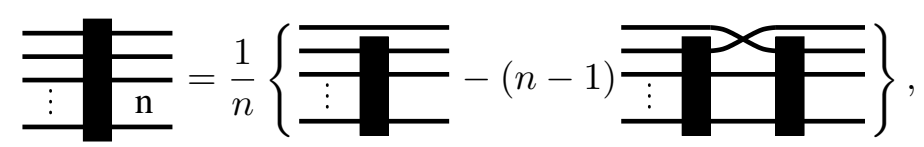

on both antisymmetrizations in (2.58), one can isolate the terms containing $\left(z_{1} \cdot \bar{z}_{1}\right)^{l_{1}}$,

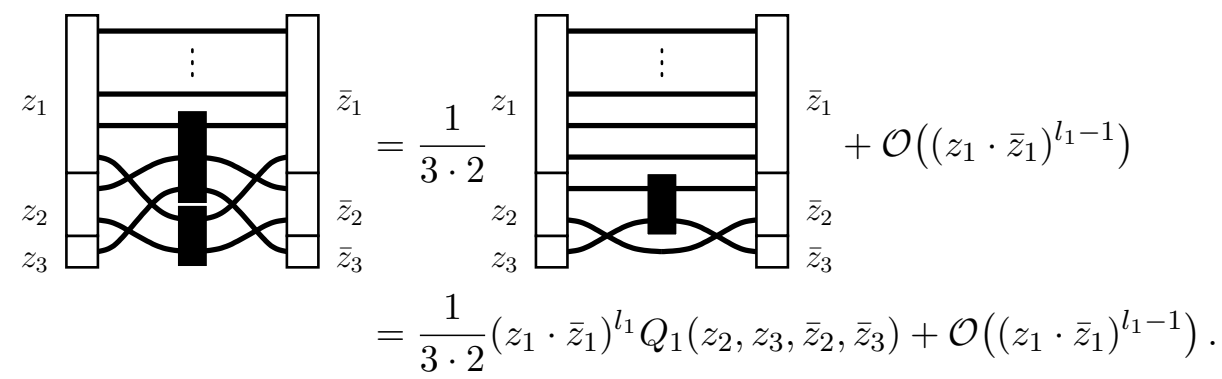

In general this factor $3 \cdot 2$ will be $\prod_{i=1}^{l_{1}} h_{i}$ and the part of the birdtrack with $z_{2}, z_{3}, \bar{z}_{2}, \bar{z}_{3}$ always matches the $z_{1}$ and $\bar{z}_{1}$ independent part of $Q_{1}\left(z_{2}, z_{3}, \bar{z}_{2}, \bar{z}_{3}\right)$. Comparing to (2.37) this means that the normalization constant appearing in the projectors is

$$
c_{\lambda}=\frac{\prod_{i=1}^{l_{1}}\left(h_{i}-1\right) ! \prod_{j=1}^{h_{1}} l_{j} !}{b_{\lambda} H(\lambda)},
$$

where $b_{\lambda}$ is the coefficient of $t^{l_{1}}$ in $f_{1}(t)$,

$$
f_{1}(t)=b_{\lambda} t^{l_{1}}+\mathcal{O}\left(t^{l_{1}-2}\right),
$$


and can be easily computed in each case using

$$
C_{n}^{(\epsilon)}(t)=\frac{(\epsilon)_{n}}{n !}(2 t)^{n}+\mathcal{O}\left(t^{n-2}\right) .
$$

Doing this for each family of projectors one finds

$$
b_{\lambda}=\frac{2^{l_{1}}\left(\frac{d}{2}-1\right){ }_{l_{1}} \prod_{i=1}^{l_{2}}\left(l_{1}+d-h_{i}+i-2\right)}{\left(l_{1}-l_{2}\right) !} .
$$

\section{Recursion relations for seed conformal blocks}

In this section recursion relations in $l_{1}$ will be read off from the integral representation of conformal blocks in the shadow formalism. The recursion relations are based on the observation that the projectors $\pi_{\lambda}$, as computed in the previous section, are linear combinations of just a few (at most $l_{2}+1$ ) different Gegenbauer polynomials,

$$
\pi_{\lambda}\left(z_{1}, z_{2}, z_{3}, \bar{z}_{1}, \bar{z}_{2}, \bar{z}_{3}\right)=c_{\lambda}\left(z_{1}^{2} \bar{z}_{1}^{2}\right)^{\frac{l_{1}}{2}} \sum_{n=l_{2}}^{\min \left(l_{1}, 2 l_{2}\right)} K_{n}^{\lambda}\left(z_{1}, z_{2}, z_{3}, \bar{z}_{1}, \bar{z}_{2}, \bar{z}_{3}\right) \mathcal{C}_{l_{1}}^{(n)}(t)
$$

where $K_{n}^{\lambda}\left(z_{1}, z_{2}, z_{3}, \bar{z}_{1}, \bar{z}_{2}, \bar{z}_{3}\right)$ does not depend on $l_{1}$, and the sum stops at $\min \left(l_{1}, 2 l_{2}\right)$ since $\mathcal{C}_{l_{1}}^{(n)}(t)=0$ for $n>l_{1}$. We showed this for the projectors to the irreps that can appear in conformal blocks of four stress-tensors by explicit computation. This fact can be used to turn the recursion relation for the Gegenbauer polynomials

$$
\left(l_{1}-n\right) \mathcal{C}_{l_{1}}^{(n)}(t)=\left(2 l_{1}+d-4\right) t \mathcal{C}_{l_{1}-1}^{(n)}(t)-\left(l_{1}+n+d-4\right) \mathcal{C}_{l_{1}-2}^{(n)}(t),
$$

into recursion relations for the conformal blocks.

\subsection{Classification of seed conformal blocks}

We will focus on conformal blocks that are unique given the irreps of the external and exchanged operators. These will be called seed conformal blocks. The origin of this terminology is the fact that conformal blocks involving three-point functions with multiple tensor structures can be derived from these seed blocks by acting with differential operators, using the method of [35].

Uniqueness of a conformal block exchanging the irrep $\lambda=\left(l_{1}, l_{2}, \ldots\right)$ in the channel $\lambda_{1} \lambda_{2} \rightarrow \lambda_{3} \lambda_{4}$, means that the three-point functions $\left\langle\lambda_{1} \lambda_{2} \lambda\right\rangle$ and $\left\langle\lambda \lambda_{3} \lambda_{4}\right\rangle$ both have a single OPE coefficient. For such combinations of irreps the tensor product contains only one symmetric tensor (or as special cases one scalar or vector), with multiplicity one

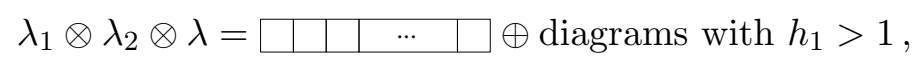

and similar for $\lambda_{3}, \lambda_{4}$. The most trivial case is when $\lambda_{1}$ and $\lambda_{2}$ are scalars, and $\lambda$ is a symmetric tensor. We will consider the case when $\lambda_{1}$ and $\lambda_{2}$ are symmetric tensors, and $\lambda$ is a mixed-symmetry tensor. In this case the lower rows of $\lambda$, which will be denoted by the Young diagram $\lambda^{-}=\left(l_{2}, l_{3}, \ldots\right)$, must be removed by contraction to $\lambda_{1}$ and $\lambda_{2}$. To understand this better let us consider three cases. 
1. $\left|\lambda_{1}\right|+\left|\lambda_{2}\right|<\left|\lambda^{-}\right| . \lambda_{1}$ and $\lambda_{2}$ do not have enough indices to remove all lower rows of $\lambda$. There is no solution to (3.3).

2. $\left|\lambda_{1}\right|+\left|\lambda_{2}\right|=\left|\lambda^{-}\right|$. If $\lambda^{-}$appears in the tensor product of $\lambda_{1}$ and $\lambda_{2}$ with multiplicity one, then (3.3) is satisfied and the symmetric tensor on the right hand side is of $\operatorname{rank} l_{1}$.

3. $\left|\lambda_{1}\right|+\left|\lambda_{2}\right|>\left|\lambda^{-}\right|$. If there are symmetric tensors in the tensor product (3.3), then there are more than one. Consider for example $\left|\lambda_{1}\right|+\left|\lambda_{2}\right|=\left|\lambda^{-}\right|+1$. After removing the lower rows of $\lambda$, there is still a tensor product of $\square$ and the remaining first row of $\lambda$, hence there will be symmetric tensors of rank $l_{1}-1$ and $l_{1}+1$.

Hence the second case is the only relevant one, leading to the necessary condition for seed conformal blocks (for $\lambda_{1}, \lambda_{2}, \lambda_{3}, \lambda_{4}$ being symmetric tensors)

$$
l_{1}=|\lambda|-\left|\lambda_{1}\right|-\left|\lambda_{2}\right|=|\lambda|-\left|\lambda_{3}\right|-\left|\lambda_{4}\right| .
$$

To see this condition in action one can consider the OPE. For example, the OPE of two vector operators has only a single term in the irreps $\lambda=\left(l_{1}, 2\right)$ or $\lambda=\left(l_{1}, 1,1\right)$, both of the form

$$
\mathcal{O}_{\Delta_{1}, \square}^{b}\left(x_{1}\right) \mathcal{O}_{\Delta_{2}, \square}^{c}\left(x_{2}\right) \underset{x_{12} \rightarrow 0}{\sim} \frac{\left(x_{12}\right)_{a_{1}} \ldots\left(x_{12}\right)_{a_{l_{1}}} \delta_{a_{l_{1}+1}}^{b} \delta_{a_{l_{1}+2}}^{c}}{\left|x_{12}\right|^{\Delta_{1}+\Delta_{2}-\Delta+l_{1}}} \mathcal{O}_{\Delta, \lambda}^{a_{1} \ldots a_{|\lambda|}}\left(x_{1}\right)+\ldots
$$

\subsection{Three-point functions}

We will use the embedding formalism and the methods to construct correlators of $[45,47]$. The embedding space is $d+2$ dimensional Minkowski space $\mathbb{R}^{d+1,1}$, where the conformal group $\mathrm{SO}(d+1,1)$ acts linearly. Capital letters will denote vectors in this space $P_{i}, Z_{i j} \in \mathbb{R}^{d+1,1}$. The second label on $Z_{i j}$ labels the different vectors required to encode mixed-symmetry tensors, e.g. $\left(z_{1}, z_{2}, z_{3}\right)$ from the previous section could be replaced by $\left(Z_{01}, Z_{02}, Z_{03}\right)$ in embedding space. Contractions of tensors can be written using derivatives acting on vectors

$$
\delta_{B_{1}}^{\left(A_{1}\right.} \ldots \delta_{B_{l_{1}}}^{\left.A_{l_{1}}\right)}=\frac{1}{l_{1} !} \partial_{Z_{i 1}}^{A_{1}} \ldots \partial_{Z_{i 1}}^{A_{l_{1}}} Z_{i 1 B_{1}} \ldots Z_{i 1 B_{l_{1}}} .
$$

Furthermore, boldface letters indicate a set of vectors that are used to encode a mixedsymmetry tensor and the corresponding sets of derivatives, normalized to include the factorial appearing in (3.6),

$$
\begin{aligned}
\mathbf{Z}_{i} & =\left(Z_{i 1}, Z_{i 2}, \ldots\right), \\
\partial_{\mathbf{Z}_{i}} & =\left(\left(l_{1} !\right)^{-1 / l_{1}} \partial_{Z_{i 1}},\left(l_{2} !\right)^{-1 / l_{2}} \partial_{Z_{i 2}}, \ldots\right) .
\end{aligned}
$$

It is convenient to consider polynomials that encode the correlators without fully implementing the symmetry and tracelessness of the operators. The full correlators can then be obtained by projecting

$$
\begin{aligned}
\left\langle\mathcal{O}_{1}\left(P_{1}, \mathbf{Z}_{1}\right) \mathcal{O}_{2}\left(P_{2}, \mathbf{Z}_{2}\right) \mathcal{O}\left(P_{0}, \mathbf{Z}_{0}\right)\right\rangle_{\text {full }}= & \pi_{\lambda_{1}}\left(\mathbf{Z}_{1}, \boldsymbol{\partial}_{\overline{\mathbf{Z}}_{1}}\right) \pi_{\lambda_{2}}\left(\mathbf{Z}_{2}, \boldsymbol{\partial}_{\overline{\mathbf{Z}}_{2}}\right) \pi_{\lambda}\left(\mathbf{Z}_{0}, \boldsymbol{\partial}_{\overline{\mathbf{Z}}_{0}}\right) \\
& \left\langle\mathcal{O}_{1}\left(P_{1}, \overline{\mathbf{Z}}_{1}\right) \mathcal{O}_{2}\left(P_{2}, \overline{\mathbf{Z}}_{2}\right) \mathcal{O}\left(P_{0}, \overline{\mathbf{Z}}_{0}\right)\right\rangle_{\text {enc }}
\end{aligned}
$$


Throughout the paper, the external operators will mostly be symmetric traceless tensors, which we will encode by a single null vector instead of projecting to a traceless tensor. Instead of (3.8) we will thus use

$$
\left\langle\mathcal{O}_{1}\left(P_{1}, Z_{1}\right) \mathcal{O}_{2}\left(P_{2}, Z_{2}\right) \mathcal{O}\left(P_{0}, \mathbf{Z}_{0}\right)\right\rangle=\pi_{\lambda}\left(\mathbf{Z}_{0}, \boldsymbol{\partial}_{\overline{\mathbf{Z}}_{0}}\right)\left\langle\mathcal{O}_{1}\left(P_{1}, Z_{1}\right) \mathcal{O}_{2}\left(P_{2}, Z_{2}\right) \mathcal{O}\left(P_{0}, \overline{\mathbf{Z}}_{0}\right)\right\rangle_{\text {enc }}
$$

where $Z_{1}^{2}=Z_{2}^{2}=0$ will always be implied. When considering conformal blocks for external operators that are either scalars, currents or stress-tensors, the three-point functions with a single tensor structure are encoded by

$$
\left\langle\mathcal{O}_{1}\left(P_{1}, Z_{1}\right) \mathcal{O}_{2}\left(P_{2}, Z_{2}\right) \mathcal{O}\left(P_{0}, \mathbf{Z}_{0}\right)\right\rangle_{\mathrm{enc}}=\frac{\left(V_{0,12}^{\left(Z_{01}\right)}\right)^{l_{1}} N_{120}^{\lambda_{1} \lambda_{2} \lambda}\left(Z_{02}, Z_{03}\right)}{\left(P_{12}\right)^{\frac{\Delta_{1}+\Delta_{2}-\Delta}{2}}\left(P_{20}\right)^{\frac{\Delta_{2}+\Delta-\Delta_{1}}{2}}\left(P_{01}\right)^{\frac{\Delta+\Delta_{1}-\Delta_{2}}{2}}},
$$

where $P_{i j}=-2 P_{i} \cdot P_{j}$ and

$$
\begin{aligned}
& N_{120}^{\bullet \bullet \square \square \square}=1, \\
& N_{120}^{\square \cdot \square \amalg \cdots \square}\left(Z_{02}\right)=H_{10}^{\left(Z_{1}, Z_{02}\right)}, \\
& N_{120}^{\square \cdot \boxplus ा \cdots \square}\left(Z_{02}\right)=\left(H_{10}^{\left(Z_{1}, Z_{02}\right)}\right)^{2}, \\
& N_{120}^{\square \square \biguplus{ }^{\cdots \cdots} \square}\left(Z_{02}\right)=H_{10}^{\left(Z_{1}, Z_{02}\right)} H_{20}^{\left(Z_{2}, Z_{02}\right)}, \\
& N_{120}^{\square \square}\left(Z_{02}, Z_{03}\right)=H_{10}^{\left(Z_{1}, Z_{02}\right)} H_{20}^{\left(Z_{2}, Z_{03}\right)} \text {, } \\
& N_{120}^{\square \square \amalg \cdots \cdots}\left(Z_{02}\right)=\left(H_{10}^{\left(Z_{1}, Z_{02}\right)}\right)^{2} H_{20}^{\left(Z_{2}, Z_{02}\right)}, \\
& N_{120}^{\square \square \bigoplus \square}\left(Z_{02}, Z_{03}\right)=\left(H_{10}^{\left(Z_{1}, Z_{02}\right)}\right)^{2} H_{20}^{\left(Z_{2}, Z_{03}\right)}, \\
& N_{120}^{\square \square \boxplus म " \square}\left(Z_{02}\right)=\left(H_{10}^{\left(Z_{1}, Z_{02}\right)}\right)^{2}\left(H_{20}^{\left(Z_{2}, Z_{02}\right)}\right)^{2}, \\
& N_{120}^{\square \square}\left(Z_{02}, Z_{03}\right)=\left(H_{10}^{\left(Z_{1}, Z_{02}\right)}\right)^{2} H_{20}^{\left(Z_{2}, Z_{02}\right)} H_{20}^{\left(Z_{2}, Z_{03}\right)} \text {, } \\
& N_{120}^{\square \square \bigoplus ~}\left(Z_{02}, Z_{03}\right)=\left(H_{10}^{\left(Z_{1}, Z_{02}\right)}\right)^{2}\left(H_{20}^{\left(Z_{2}, Z_{03}\right)}\right)^{2},
\end{aligned}
$$

with

$$
\begin{aligned}
H_{i j}^{\left(Z_{i}, Z_{j}\right)} & =\frac{\left(Z_{i} \cdot Z_{j}\right)\left(P_{i} \cdot P_{j}\right)-\left(P_{j} \cdot Z_{i}\right)\left(P_{i} \cdot Z_{j}\right)}{P_{i} \cdot P_{j}}, \\
V_{i, j k}^{(Z)} & =\frac{\left(P_{j} \cdot Z\right)\left(P_{i} \cdot P_{k}\right)-\left(P_{j} \cdot P_{i}\right)\left(Z \cdot P_{k}\right)}{\sqrt{-2\left(P_{i} \cdot P_{j}\right)\left(P_{i} \cdot P_{k}\right)\left(P_{j} \cdot P_{k}\right)}} .
\end{aligned}
$$

\subsection{Recursion relations from the shadow formalism}

Next we recall the formula for the conformal partial wave in the shadow formalism (an overview of the formalism in the case of mixed-symmetry tensors can be found in section 5 of [45]). When using the three-point functions defined above, the exchanged representation 
must be projected to an irreducible representation

$$
\begin{aligned}
W_{\Delta, \lambda}^{\lambda_{1} \lambda_{2} \lambda_{3} \lambda_{4}}= & \frac{1}{\mathcal{S}_{\tilde{\Delta} \Delta_{34}}^{\lambda}} \int D^{d} P_{0}\left\langle\mathcal{O}_{1}\left(P_{1}, Z_{1}\right) \mathcal{O}_{2}\left(P_{2}, Z_{2}\right) \mathcal{O}\left(P_{0}, \boldsymbol{\partial}_{\mathbf{Z}_{0}}\right)\right\rangle_{\mathrm{enc}} \\
& \left.\pi_{\lambda}\left(\mathbf{Z}_{0}, \boldsymbol{\partial}_{\overline{\mathbf{Z}}_{0}}\right)\left\langle\mathcal{O}_{3}\left(P_{3} ; Z_{3}\right) \mathcal{O}_{4}\left(P_{4} ; Z_{4}\right) \mathcal{O}\left(P_{0}, \overline{\mathbf{Z}}_{0}\right)\right\rangle_{\text {enc }}\right|_{\Delta \rightarrow \tilde{\Delta}} .
\end{aligned}
$$

Here $\tilde{\Delta}=d-\Delta$ is the conformal dimension of the shadow operator $\tilde{\mathcal{O}}$ and $\mathcal{S}_{\tilde{\Delta} \Delta_{34}}^{\lambda}=$ $\left.\mathcal{S}_{\Delta \Delta_{34}}^{\lambda}\right|_{\Delta \rightarrow \tilde{\Delta}}$ is the constant that occurs when an operator in a three-point function is replaced by its shadow

$$
\mathcal{S}_{\Delta \Delta_{34}}^{\lambda}=\frac{\left\langle\mathcal{O}_{3}\left(P_{3} ; \mathbf{Z}_{3}\right) \mathcal{O}_{4}\left(P_{4} ; \mathbf{Z}_{4}\right) \tilde{\mathcal{O}}\left(P_{0} ; \mathbf{Z}_{0}\right)\right\rangle}{\left.\left\langle\mathcal{O}_{3}\left(P_{3} ; \mathbf{Z}_{3}\right) \mathcal{O}_{4}\left(P_{4} ; \mathbf{Z}_{4}\right) \mathcal{O}\left(P_{0} ; \mathbf{Z}_{0}\right)\right\rangle\right|_{\Delta \rightarrow \tilde{\Delta}}}
$$

It is computed in appendix D for any three-point function that can appear in a seed conformal block, with the result

$$
\mathcal{S}_{\tilde{\Delta} \Delta_{34}}^{\lambda}=\pi^{d / 2} \prod_{i=1}^{l_{1}}\left(\tilde{\Delta}-h_{i}+i-1\right) \frac{\Gamma\left(\tilde{\Delta}-\frac{d}{2}\right) \Gamma\left(\frac{1}{2}\left(\Delta+\Delta_{34}+l_{1}\right)\right) \Gamma\left(\frac{1}{2}\left(\Delta-\Delta_{34}+l_{1}\right)\right)}{\Gamma\left(\Delta+l_{1}\right) \Gamma\left(\frac{1}{2}\left(\tilde{\Delta}+\Delta_{34}+l_{1}\right)\right) \Gamma\left(\frac{1}{2}\left(\tilde{\Delta}-\Delta_{34}+l_{1}\right)\right)} .
$$

We start by inserting the expressions for the three-point functions (3.10) into (3.13), to find

$$
\begin{aligned}
& W_{\Delta, \lambda}^{\lambda_{1} \lambda_{2} \lambda_{3} \lambda_{4}}=\frac{1}{\mathcal{S}_{\tilde{\Delta} \Delta_{34}}^{\lambda}\left(l_{2} ! l_{3} !\right)^{2}} \\
& \quad \int D^{d} P_{0} \frac{N_{120}^{\lambda_{1} \lambda_{2} \lambda}\left(\partial_{Z_{02}}, \partial_{Z_{03}}\right) \pi_{\lambda}\left(\partial_{Z_{01}} V_{0,12}^{\left(Z_{01}\right)}, Z_{02}, Z_{03}, \partial_{\bar{Z}_{01}} V_{0,34}^{\left(\bar{Z}_{01}\right)}, \partial_{\bar{Z}_{02}}, \partial_{\bar{Z}_{03}}\right) N_{340}^{\lambda_{3} \lambda_{4} \lambda}\left(\bar{Z}_{02}, \bar{Z}_{03}\right)}{\left(P_{01}\right)^{\frac{\Delta+\Delta_{12}}{2}}\left(P_{20}\right)^{\frac{\Delta-\Delta_{12}}{2}}\left(P_{03}\right)^{\frac{\Delta}{\Delta+\Delta_{34}}}\left(P_{40}\right)^{\frac{\tilde{\Delta}-\Delta_{34}}{2}}} .
\end{aligned}
$$

Next we wish to insert the expression (3.1) for the projector $\pi_{\lambda}$. The factor $\left(z_{1}^{2} \bar{z}_{1}^{2}\right)^{\frac{l_{1}}{2}}$ in (3.1) is equal to 1 due to $\left(\partial_{Z_{01}} V_{0,12}^{\left(Z_{01}\right)}\right)^{2}=\left(\partial_{\bar{Z}_{01}} V_{0,34}^{\left(\bar{Z}_{01}\right)}\right)^{2}=1$. Since the factors $\mathcal{S}_{\tilde{\Delta} \Delta_{34}}^{\lambda}$ appearing in (3.16) and $c_{\lambda}$ in (3.1) depend on $l_{1}$, it is convenient to use the following normalization for the conformal blocks ${ }^{5}$

$$
G_{\Delta, \lambda}^{\lambda_{1} \lambda_{2} \lambda_{3} \lambda_{4}}=\frac{\mathcal{S}_{\tilde{\Delta} \Delta_{34}}^{\lambda}}{c_{\lambda}}\left(P_{12}\right)^{\frac{\Delta}{2}}\left(P_{34}\right)^{\frac{\tilde{\Delta}}{2}}\left(\frac{P_{14}}{P_{24}}\right)^{\frac{\Delta_{12}}{2}}\left(\frac{P_{13}}{P_{14}}\right)^{\frac{\Delta_{34}}{2}} W_{\Delta, \lambda}^{\lambda_{1} \lambda_{2} \lambda_{3} \lambda_{4}}
$$

By inserting (3.1) into (3.16) we can now write the conformal block as a sum of functions that only depend on a single Gegenbauer polynomial each,

$$
G_{\Delta, \lambda}^{\lambda_{1} \lambda_{2} \lambda_{3} \lambda_{4}}=\sum_{n=l_{2}}^{\min \left(l_{1}, 2 l_{2}\right)} F_{\Delta, \lambda, n}^{\lambda_{1} \lambda_{2} \lambda_{3} \lambda_{4}}\left(\Delta_{12}, \Delta_{34}\right)
$$

\footnotetext{
${ }^{5}$ As explained in [39], to obtain the conformal block one needs to perform a monodromy projection of the integral (3.13). This is assumed but not written explicitly because it is not important for the present discussion.
} 
which are defined as

$$
\begin{aligned}
& F_{\Delta, \lambda, n}^{\lambda_{1} \lambda_{2} \lambda_{3} \lambda_{4}}\left(\Delta_{12}, \Delta_{34}\right)= \\
& \frac{1}{\left(l_{2} ! l_{3} !\right)^{2}}\left(P_{12}\right)^{\frac{\Delta}{2}}\left(P_{34}\right)^{\frac{\tilde{\Delta}}{2}}\left(\frac{P_{14}}{P_{24}}\right)^{\frac{\Delta_{12}}{2}}\left(\frac{P_{13}}{P_{14}}\right)^{\frac{\Delta_{34}}{2}} \int D^{d} P_{0} \\
& \frac{N_{120}^{\lambda_{1} \lambda_{2} \lambda}\left(\partial_{Z_{02}}, \partial_{Z_{03}}\right) K_{n}^{\lambda}\left(\partial_{Z_{01}} V_{0,12}^{\left(Z_{01}\right)}, Z_{02}, Z_{03}, \partial_{\bar{Z}_{01}} V_{0,34}^{\left(\bar{Z}_{01}\right)}, \partial_{\bar{Z}_{02}}, \partial_{\bar{Z}_{03}}\right) N_{340}^{\lambda_{3} \lambda_{4} \lambda}\left(\bar{Z}_{02}, \bar{Z}_{03}\right) \mathcal{C}_{l_{1}}^{(n)}(t)}{\left(P_{01}\right)^{\frac{\Delta+\Delta_{12}}{2}}\left(P_{20}\right)^{\frac{\Delta-\Delta_{12}}{2}}\left(P_{03}\right)^{\frac{\tilde{\Delta}+\Delta_{34}}{2}}\left(P_{40}\right)^{\frac{\tilde{\Delta}-\Delta_{34}}{2}}},
\end{aligned}
$$

where

$$
t=\frac{1}{2 \sqrt{P_{12} P_{34}}}\left(\sqrt{\frac{P_{02} P_{03}}{P_{01} P_{04}}} P_{14}-\sqrt{\frac{P_{02} P_{04}}{P_{01} P_{03}}} P_{13}-\sqrt{\frac{P_{01} P_{03}}{P_{02} P_{04}}} P_{24}+\sqrt{\frac{P_{01} P_{04}}{P_{02} P_{03}}} P_{23}\right) .
$$

The Gegenbauer recursion relation (3.2) implies the following recursion relations for each of these functions

$$
\begin{aligned}
& \left(l_{1}-n\right) F_{\Delta,\left(l_{1}, l_{2}, l_{3}\right), n}^{\lambda_{1} \lambda_{2} \lambda_{3} \lambda_{4}}\left(\Delta_{12}, \Delta_{34}\right)= \\
& \left(l_{1}+\frac{d}{2}-2\right) u^{-\frac{1}{2}}\left(F_{\Delta,\left(l_{1}-1, l_{2}, l_{3}\right), n}^{\lambda_{1} \lambda_{2} \lambda_{3} \lambda_{4}}\left(\Delta_{12}+1, \Delta_{34}-1\right)-F_{\Delta,\left(l_{1}-1, l_{2}, l_{3}\right), n}^{\lambda_{1} \lambda_{2} \lambda_{3} \lambda_{4}}\left(\Delta_{12}+1, \Delta_{34}+1\right)\right. \\
& \left.\quad-F_{\Delta,\left(l_{1}-1, l_{2}, l_{3}\right), n}^{\lambda_{1} \lambda_{2} \lambda_{3} \lambda_{4}}\left(\Delta_{12}-1, \Delta_{34}-1\right)+v F_{\Delta,\left(l_{1}-1, l_{2}, l_{3}\right), n}^{\lambda_{1} \lambda_{2} \lambda_{3} \lambda_{4}}\left(\Delta_{12}-1, \Delta_{34}+1\right)\right) \\
& -\left(l_{1}+n+d-4\right) F_{\Delta,\left(l_{1}-2, l_{2}, l_{3}\right), n}^{\left.\lambda_{1} \lambda_{2} \lambda_{3} \lambda_{12}, \Delta_{34}\right),}
\end{aligned}
$$

where $u=\frac{P_{12} P_{34}}{P_{13} P_{24}}, v=\frac{P_{14} P_{23}}{P_{13} P_{24}}$ are the conformal cross-ratios. To use the recursion relations, it is only necessary to know the conformal blocks for $l_{1}=l_{2}$ up to $l_{1}=2 l_{2}$ as seeds. Once these are known, one can forget about the conformal integrals that were used for the derivation of the recursion relations and even the precise form of the functions $K_{n}^{\lambda}$. Any family of conformal blocks can be mapped to the functions $F_{\Delta, \lambda, i}^{\lambda_{1} \lambda_{2} \lambda_{3} \lambda_{4}}$ using that

$$
\begin{aligned}
G_{\Delta,\left(l_{2}, l_{2}, l_{3}\right)}^{\lambda_{1} \lambda_{2} \lambda_{3} \lambda_{4}} & =F_{\Delta,\left(l_{2}, l_{2}, l_{3}\right), l_{2}}^{\lambda_{1} \lambda_{2} \lambda_{2} \lambda_{4}}, \\
G_{\Delta,\left(l_{2}+1, l_{2}, l_{3}\right)}^{\lambda_{1} \lambda_{2} \lambda_{3} \lambda_{3}} & =F_{\Delta,\left(l_{2}+1, l_{2}, l_{3}\right), l_{2}}^{\lambda_{1} \lambda_{2} \lambda_{4}}+F_{\Delta,\left(l_{2}+1, l_{2}, l_{3}\right), l_{2}+1}^{\lambda_{1} \lambda_{2} \lambda_{3} \lambda_{4}}, \\
G_{\Delta,\left(l_{2}+2, l_{2}, l_{3}\right)}^{\lambda_{1} \lambda_{2} \lambda_{3} \lambda_{4}} & =F_{\Delta,\left(l_{2}+2, l_{2}, l_{3}\right), l_{2}}^{\lambda_{1} \lambda_{2} \lambda_{3} \lambda_{4}}+F_{\Delta,\left(l_{2}+2, l_{2}, l_{3}\right), l_{2}+1}^{\lambda_{1} \lambda_{2} \lambda_{3} \lambda_{4}}+F_{\Delta,\left(l_{2}+2, l_{2}, l_{3}\right), l_{2}+2}^{\lambda_{1} \lambda_{2} \lambda_{3} \lambda_{4}},
\end{aligned}
$$

Hence the conformal blocks for $l_{1}=l_{2}, \ldots, 2 l_{2}$ can be used in conjunction with the recursion relation to compute the seeds

$$
F_{\Delta,\left(n, l_{2}, l_{3}\right), n}, \quad n=l_{2}, \ldots, 2 l_{2} .
$$

For example, the conformal blocks for exchange of $\boxminus$ and $\boxminus$ act as seeds for the family $\square+\cdots$ in the following way

$$
\begin{aligned}
& G_{\Delta,(1,1)}^{\square \bullet \bullet \bullet}=F_{\Delta,(1,1), 1}^{\square \bullet \square \bullet} \\
& \downarrow \\
& G_{\Delta,(2,1)}^{\square \bullet \bullet}=F_{\Delta,(2,1), 1}^{\square \bullet}+F_{\Delta,(2,1), 2}^{\square \bullet \bullet \bullet} \\
& \downarrow \quad \downarrow \\
& G_{\Delta,(3,1)}^{\square \bullet}=F_{\Delta,(3,1), 1}^{\square \bullet \square \bullet}+F_{\Delta,(3,1), 2}^{\square \bullet \bullet \bullet}
\end{aligned}
$$


The recursion relation (3.21) allows us to move down along the arrows. Note that in order for the conformal blocks to satisfy the stated recursion relations, it is crucial that their normalization depends on $l_{1}, \Delta_{12}$ and $\Delta_{34}$ as defined in (3.17). A good method to compare normalizations for conformal blocks obtained via different methods is to consider the OPE limit, which is done for the partial wave (3.13) in appendix E.

\subsection{Solution of the recursion relation in terms of scalar conformal blocks}

For $n=0$ the recursion relation (3.21) is equivalent to the one for scalar conformal blocks that was solved for $d=2,4$ in [40]. The new parameter $n$ can be removed from the prefactors by using the variables $l_{1}^{\prime}=l_{1}-n$ and $d^{\prime}=d+2 n$,

$$
\begin{aligned}
& l_{1}^{\prime} F_{\Delta,\left(l_{1}, l_{2}, l_{3}\right), n}^{\lambda_{1} \lambda_{2} \lambda_{3} \lambda_{4}}\left(\Delta_{12}, \Delta_{34}\right)= \\
& \left(l_{1}^{\prime}+\frac{d^{\prime}}{2}-2\right) u^{-\frac{1}{2}}\left(F_{\Delta,\left(l_{1}-1, l_{2}, l_{3}\right), n}^{\lambda_{1} \lambda_{2} \lambda_{3} \lambda_{4}}\left(\Delta_{12}+1, \Delta_{34}-1\right)-F_{\Delta,\left(l_{1}-1, l_{2}, l_{3}\right), n}^{\lambda_{1} \lambda_{2} \lambda_{3} \lambda_{4}}\left(\Delta_{12}+1, \Delta_{34}+1\right)\right. \\
& \left.\quad-F_{\left.\Delta, l_{1}-1, l_{2}, l_{3}\right), n}^{\lambda_{1} \lambda_{2} \lambda_{3} \lambda_{4}}\left(\Delta_{12}-1, \Delta_{34}-1\right)+v F_{\Delta,\left(l_{1}-1, l_{2}, l_{3}\right), n}^{\lambda_{1} \lambda_{2} \lambda_{3} \lambda_{4}}\left(\Delta_{12}-1, \Delta_{34}+1\right)\right) \\
& \quad\left(l_{1}^{\prime}+d^{\prime}-4\right) F_{\Delta,\left(l_{1}-2, l_{2}, l_{3}\right), n}^{\lambda_{1} \lambda_{2} \lambda_{3} \lambda_{1}}\left(\Delta_{12}, \Delta_{34}\right) .
\end{aligned}
$$

This implies that the scalar conformal block in $d^{\prime}$ dimensions

$$
G_{\Delta, l_{1}^{\prime}, d^{\prime}}^{\Delta_{12}, \Delta_{34}}(u, v) \equiv G_{\Delta,\left(l_{1}^{\prime}\right)}^{\bullet \bullet \bullet \bullet ~ i n ~} d^{\prime} \text { dimensions },
$$

is a solution of the recurrence relation for seed conformal blocks. Maybe this can be used to solve the recursion relations in terms of known functions. While we do not know if this is actually possible, assume for a moment that the seeds for the recursion relation can be written in terms of scalar conformal blocks as

$$
F_{\Delta,\left(n, l_{2}, l_{3}\right), n}=\sum_{i, j, k} f_{i, j, k} G_{\Delta+k, 0, d+2 n}^{\Delta_{12}+i, \Delta_{34}+j}(u, v), \quad n=l_{2}, \ldots, 2 l_{2} .
$$

We allowed for arbitrary shifts $i, j, k$ in the three parameters $\Delta_{12}, \Delta_{34}$ and $\Delta$ and functions $f_{i, j, k}$ that do not influence the recursion relation, i.e. $f_{i, j, k}$ can depend on $\Delta, u, v$ and the polarizations of external operators, but not on $l_{1}, d, \Delta_{12}$ or $\Delta_{34}$. Then the result for arbitrary $l_{1}$ would be the same linear combination of scalar conformal blocks with appropriately increased spin $l_{1}$,

$$
F_{\Delta,\left(l_{1}, l_{2}, l_{3}\right), n}=\sum_{i, j, k} f_{i, j, k} G_{\Delta+k, l_{1}-n, d+2 n}^{\Delta_{12}+i, \Delta_{34}+j}(u, v) .
$$

In $[39,45]$ the two initial blocks of the family $\left(l_{1}, 1\right)$ were computed explicitly in terms of conformal blocks with $l_{1}=0$ in higher dimensions, however the block $G_{\Delta,(1,1)}^{\square \bullet}=F_{\Delta,(1,1), 1}$ was given in terms of scalar blocks in 8 dimensions instead of the $d^{\prime}=6$ required by (3.27). While the simple relation to scalar conformal blocks assumed in (3.27) might not be true, it is likely that the recursion relations should be solvable in terms of functions similar to scalar conformal blocks in higher dimensions. This correspondence was also observed in the recent paper [48], where the mixed-symmetry seed conformal blocks in four dimensions were computed using a twistor formalism. 


\subsection{Recursion relation for radial coordinates}

Here we will discuss how to use these recursion relations for expansions of conformal blocks in the radial coordinates $(r, \eta)$ of [49-51]. These coordinates are related to the cross-ratios $(u, v)$ by

$$
u=\frac{16 r^{2}}{\left(r^{2}+2 \eta r+1\right)^{2}}, \quad v=\frac{\left(r^{2}-2 \eta r+1\right)^{2}}{\left(r^{2}+2 \eta r+1\right)^{2}},
$$

and one typically considers expansions of the conformal blocks up to some order $m$ in $r$

$$
\begin{aligned}
G_{\Delta, \lambda}^{\lambda_{1} \lambda_{2} \lambda_{3} \lambda_{4}}(r) & =\sum_{m^{\prime}=0}^{m} r^{\Delta+m^{\prime}} H_{m^{\prime}}+\mathcal{O}\left(r^{\Delta+m+1}\right) \\
& \equiv G_{\Delta, \lambda}^{\lambda_{1} \lambda_{2} \lambda_{3} \lambda_{4}}(r, m)+\mathcal{O}\left(r^{\Delta+m+1}\right)
\end{aligned}
$$

For such expansions the recursion relations can be used by splitting them into contributions which satisfy the different recursion relations, as in (3.18)

$$
G_{\Delta, \lambda}^{\lambda_{1} \lambda_{2} \lambda_{3} \lambda_{4}}(r, m)=\sum_{n=l_{2}}^{2 l_{2}} F_{\Delta, \lambda, n}^{\lambda_{1} \lambda_{2} \lambda_{3} \lambda_{4}}\left(\Delta_{12}, \Delta_{34} ; r, m\right) .
$$

The identification of the different parts of the conformal blocks can be performed as illustrated in the diagram (3.24), by starting at the lowest allowed $l_{1}$ and using the recursion to find the term that has to be subtracted from the next conformal block to get the seed for the next recursion relation. To derive the recursion relations in $(r, \eta)$ one has to expand $u^{-\frac{1}{2}}$ and $v$ in $r$

$$
u^{-\frac{1}{2}}=\frac{1}{4 r}+\frac{\eta}{2}+\frac{r}{4}, \quad v=1+\mathcal{O}(r) .
$$

Unfortunately the appearance of the term of order $r^{-1}$ means that the recursion relations decrease the order of the expansion in $r$. They read

$$
\begin{aligned}
& \left(l_{1}-n\right) F_{\Delta,\left(l_{1}, l_{2}, l_{3}\right), n}^{\lambda_{1} \lambda_{2} \lambda_{2} \lambda_{4}}\left(\Delta_{12}, \Delta_{34} ; r, m\right)= \\
& \left(l_{1}+\frac{d}{2}-2\right)\left(\frac{1}{4 r}+\frac{\eta}{2}+\frac{r}{4}\right)\left(F_{\Delta,\left(l_{1}-1, l_{2}, l_{3}\right), n}^{\lambda_{1} \lambda_{2} \lambda_{3} \lambda_{4}}\left(\Delta_{12}+1, \Delta_{34}-1 ; r, m+1\right)\right. \\
& \quad-F_{\Delta, l_{1} \lambda_{2} \lambda_{3} \lambda_{4}}^{\lambda_{\left.1, l_{l}, l_{3}\right), n}}\left(\Delta_{12}+1, \Delta_{34}+1 ; r, m+1\right)-F_{\Delta,\left(l_{1}-1, l_{2}, l_{3}\right), n}^{\lambda_{1} \lambda_{2} \lambda_{3} \lambda_{4}}\left(\Delta_{12}-1, \Delta_{34}-1 ; r, m+1\right) \\
& \left.\quad+v F_{\Delta,\left(l_{1}-1, l_{2}, l_{3}\right), n}^{\lambda_{1} \lambda_{2} \lambda_{3} \lambda_{4}}\left(\Delta_{12}-1, \Delta_{34}+1 ; r, m+1\right)\right)-\left(l_{1}+n+d-4\right) F_{\Delta,\left(l_{1}-2, l_{2}, l_{3}\right), n}^{\lambda_{1} \lambda_{2} \lambda_{3} \lambda_{4}}\left(\Delta_{12}, \Delta_{34} ; r, m\right) .
\end{aligned}
$$

These relations were checked with the $r$ expansions from [51] for the case of two external vectors and exchange of the irrep $\square$, which is depicted in the diagram (3.24). To this end the normalizations were matched in the OPE limit.

\section{Concluding remarks}

In this paper the projectors to traceless mixed-symmetry tensors that appear in the correlator of four stress tensors were derived in terms of Gegenbauer polynomials. Knowledge of the explicit form of the projectors led us to a single universal recursion relation in $l_{1}$ for seed conformal blocks, given by (3.21). Interestingly, the existence of the recursion 
relation does not rely on the complete expressions for the projectors, but only requires that their dependence on $l_{1}$ is according to (3.1). Of course this implies that, to show that the recursion relation is truly universal (i.e. holds for any seed conformal block of bosonic operators), it is enough to prove that all projectors to traceless mixed-symmetry tensors can be written as in (3.1).

In order for the conformal blocks to obey the recursion relation, it is required that they are normalized in a particular way. In particular, the normalization constant of the projector as well as the normalization of the shadow operator need to be canceled from the integral expression of the conformal partial wave. To this end the normalization of the shadow was computed. Furthermore, the OPE limit of the shadow integral was analyzed to allow for comparisons to other results.

Another remark concerns the solution of the recursion relations. The new recursion relations (3.21) are generalizations of the recursion relation for scalar conformal blocks of [38] with a new parameter $n$. This new parameter can be absorbed into $l_{1}$ and $d$ by using shifted parameters

$$
l_{1}^{\prime}=l_{1}-n, \quad d^{\prime}=d+2 n .
$$

As a result the recursion relations for general seed conformal blocks are solved by scalar conformal blocks in higher dimensions, suggesting that seed conformal blocks can generally be expressed in terms of such scalar blocks. A similar correspondence was also observed in the recent paper [48] for the case $d=4$.

Beside this application, we want to stress that the projectors actually play a very important role in CFTs. The most basic example in which they appear is the two point functions of mixed-symmetry operators,

$$
\left\langle\mathcal{O}_{\Delta, \lambda}^{a_{1} \ldots a_{|\lambda|}}(x) \mathcal{O}_{\Delta, \lambda}^{b_{1} \ldots b_{|\lambda|}}(0)\right\rangle=|x|^{-2 \Delta} \pi_{\lambda}^{c_{1} \ldots c_{|\lambda|}, b_{1} \ldots b_{|\lambda|}} \prod_{i=1}^{|\lambda|}\left(\delta_{c_{i}}^{a_{i}}-2 \frac{x^{a_{i}} x_{c_{i}}}{x^{2}}\right) .
$$

Moreover, the projector $\pi_{\lambda}$ is a necessary ingredient to compute a conformal block for the exchange of an irrep $\lambda$. For example, the OPE limit of any seed conformal block with external operators $\mathcal{O}_{i}$ of spins $\ell_{i}$ is always written (here without being precise on the placement of the indices on the $\pi_{\ell_{i}}$ ) in terms of the two-point function of the exchanged operator as

$$
\frac{x_{12 a_{1}} \ldots x_{12 a_{l_{1}}}\left(\pi_{\ell_{1}} \pi_{\ell_{2}}\right)_{a_{l_{1}+1} \ldots a_{|\lambda|}}^{c_{1} \ldots \ell_{\ell_{1}} e_{1} \ldots e_{\ell_{2}}} x_{34 b_{1}} \ldots x_{34 b_{l_{1}}}\left(\pi_{\ell_{3}} \pi_{\ell_{4}}\right)_{b_{l_{1}+1} \ldots b_{|\lambda|}}^{f_{1} \ldots f_{\ell_{3}} g_{1} \ldots g_{\ell_{4}}}\left\langle\mathcal{O}_{\Delta, \lambda}^{a_{1} \ldots a_{|\lambda|}}\left(x_{2}\right) \mathcal{O}_{\Delta, \lambda}^{b_{1} \ldots b_{|\lambda|}}\left(x_{4}\right)\right\rangle}{\left|x_{12}\right|^{\Delta_{1}+\Delta_{2}-\Delta+l_{1}}\left|x_{34}\right|^{\Delta_{3}+\Delta_{4}-\Delta+l_{1}}}
$$

therefore it involves the projector $\pi_{\lambda}$ (see appendix E). Of course, also the leading OPE of any other conformal block is written in terms of the projectors since it can be generated by acting with some derivatives on the seed block. From this remark it is clear that the knowledge of $\pi_{\lambda}$ is needed to compute conformal blocks from their radial coordinate expansion [49-51], since the leading term of the expansion is the leading OPE.

To compute conformal blocks with the shadow formalism it is crucial to know the form of $\pi_{\lambda}$ as well, since it appears explicitly in the shadow integral. In this paper we expressed $\pi_{\lambda}$ in terms of derivatives of the Gegenbauer polynomial, which encodes the 
projector to traceless symmetric irreps. Therefore, it should be possible to compute any family of seed conformal blocks (for generic $l_{1}$ ) in terms of scalar conformal blocks by a direct computation. To achieve this, it is enough to rewrite the integrand of the shadow integral of the seed conformal block (3.16) in terms of derivatives of integrands of scalar conformal blocks. The general result for seed conformal blocks of the family $\lambda=\left(l_{1}, 1\right)$ was obtained in this way in [42].

\section{Acknowledgments}

This research received funding from the [European Union] 7th Framework Programme (Marie Curie Actions) under grant agreements No 269217 and 317089 (GATIS), and from the research grant CERN/FIS-NUC/0045/2015. T.H. was supported by the German Science Foundation (DFG) within the Collaborative Research Center 676 "Particles, Strings and the Early Universe". The work of E.T. has been supported by the Portuguese Fundacão para a Ciência e a Tecnologia (FCT) through the fellowship SFRH/BD/51984/2012. His research was partially supported by Perimeter Institute for Theoretical Physics. Research at Perimeter Institute is supported by the Government of Canada through Industry Canada and by the Province of Ontario though the Ministry of Economic Development \& innovation.

\section{A A mixed-symmetry differential operator}

In this section we find an alternative way to generate the projectors into traceless mixedsymmetry tensors for Young diagrams with two rows. The main idea is to generalize the result of [52], where the differential operator

$$
D_{z}^{a}=\left(\frac{d}{2}-1+z \cdot \frac{\partial}{\partial z}\right) \frac{\partial}{\partial z_{a}}-\frac{1}{2} z^{a} \frac{\partial^{2}}{\partial z \cdot \partial z},
$$

was defined in order to generate projectors to traceless symmetric tensor representations

$$
\pi_{(l)}^{a_{1} \ldots a_{l}, b_{1} \ldots b_{l}}=\frac{1}{l !\left(\frac{d}{2}-1\right)_{l}} D_{z}^{a_{1}} \ldots D_{z}^{a_{l}} z^{b_{1}} \ldots z^{b_{l}} .
$$

One can construct this operator by looking for an operator of weight -1 in $z$ that preserves the space defined by $z^{2}=0$, i.e.

$$
D_{z}^{a}\left(z^{2} f(z)\right)=\mathcal{O}\left(z^{2}\right)
$$

This ensures tracelessness of the expression (A.2), since contracting with $\delta_{b_{1} b_{2}}$ and acting with all the operators yields something that is $\mathcal{O}\left(z^{2}\right)$ and of degree zero in $z$, hence 0 .

A generalized differential operator for Young diagrams with two rows should generate the projectors into traceless mixed-symmetry tensors in a similar way by acting on a combination of two different vectors

$$
\begin{gathered}
\pi_{\lambda}^{\left[a_{1} a_{2}\right] \ldots\left[a_{2 l_{2}-1} a_{2 l_{2}}\right] a_{2 l_{2}+1} \ldots a_{l_{1}+l_{2},},\left[b_{1} b_{2}\right] \ldots\left[b_{2 l_{2}-1} b_{2 l_{2}}\right] b_{2 l_{2}+1} \ldots b_{l_{1}+l_{2}}}= \\
\mathcal{N}_{l_{1}, l_{2}} D_{1}^{\left[a_{1}\right.} D_{2}^{\left.a_{2}\right]} \ldots D_{1}^{\left[a_{2 l_{2}-1}\right.} D_{2}^{\left.a_{2 l_{2}}\right]} D_{1}^{a_{2 l_{2}+1}} \ldots D_{1}^{a_{l_{1}+l_{2}}} z_{1}^{\left[b_{1}\right.} z_{2}^{\left.b_{2}\right]} \ldots z_{1}^{\left[b_{2 l_{2}-1}\right.} z_{2}^{\left.b_{2 l_{2}}\right]} z_{1}^{b_{2 l_{2}+1}} \ldots z_{1}^{b_{l_{1}+l_{2}}} .
\end{gathered}
$$


Clearly this expression is Young symmetrized (in the antisymmetric representation) and traceless, provided the differential operators $D_{1}$ and $D_{2}$ preserve the space defined by $z_{1}^{2}=0, z_{2}^{2}=0$ and $z_{1} \cdot z_{2}=0$, namely

$$
\begin{aligned}
D_{1,2}^{a}\left(z_{1}^{2} f\left(z_{1}, z_{2}\right)\right) & =\mathcal{O}\left(z_{1}^{2}, z_{1} \cdot z_{2}, z_{2}^{2}\right), \\
D_{1,2}^{a}\left(z_{2}^{2} f\left(z_{1}, z_{2}\right)\right) & =\mathcal{O}\left(z_{1}^{2}, z_{1} \cdot z_{2}, z_{2}^{2}\right), \\
D_{1,2}^{a}\left(z_{1} \cdot z_{2} f\left(z_{1}, z_{2}\right)\right) & =\mathcal{O}\left(z_{1}^{2}, z_{1} \cdot z_{2}, z_{2}^{2}\right) .
\end{aligned}
$$

Furthermore, $D_{1}$ must have weight -1 in $z_{1}$ and 0 in $z_{2}$, and $D_{2}$ must have weight 0 in $z_{1}$ and -1 in $z_{2}$. These requirements fix the operators completely as ${ }^{6}$

$$
\begin{aligned}
D_{1}^{a} & \equiv D_{z_{1}, z_{2}}^{a} \equiv d_{00} \partial_{z_{1}}^{a}+d_{-11} \partial_{z_{2}}^{a}+z_{1}^{a} d_{-20}+z_{2}^{a} d_{-1-1}, \\
D_{2}^{a} & \equiv D_{z_{2}, z_{1}}^{a} .
\end{aligned}
$$

where $d_{m n}$ are differential operators with weight $m$ in the variable $z_{1}$ and $n$ in the variable $z_{2}$, defined by

$$
\begin{aligned}
d_{00} & \equiv\left(1-\frac{d}{2}\right)\left[(d-3)+3\left(z_{1} \cdot \partial_{z_{1}}\right)+\left(z_{2} \cdot \partial_{z_{2}}\right)\right]-\left(z_{1} \cdot \partial_{z_{1}}\right)\left(z_{2} \cdot \partial_{z_{2}}\right)-z_{1}^{a}\left(z_{1} \cdot \partial_{z_{1}}\right) \partial_{z_{1}} a \\
d_{-20} & \equiv \frac{1}{2}\left[2\left(\frac{d}{2}-1\right)+\left(z_{2} \cdot \partial_{z_{2}}\right)+\left(z_{1} \cdot \partial_{z_{1}}\right)\right]\left(\partial_{z_{1}} \cdot \partial_{z_{1}}\right), \\
d_{-11} & \equiv-(d-2)\left(z_{2} \cdot \partial_{z_{1}}\right)-\left(z_{2} \cdot \partial_{z_{1}}\right)\left(z_{2} \cdot \partial_{z_{2}}\right)-\left(z_{1} \cdot \partial_{z_{1}}\right)\left(z_{2} \cdot \partial_{z_{1}}\right), \\
d_{-1-1} & \equiv\left[\left(\frac{d}{2}-1\right)+\left(z_{1} \cdot \partial_{z_{1}}\right)\right]\left(\partial_{z_{1}} \cdot \partial_{z_{2}}\right)+\frac{1}{2}\left[\left(z_{2} \cdot \partial_{z_{1}}\right)\left(\partial_{z_{2}} \cdot \partial_{z_{2}}\right)-\left(z_{1} \cdot \partial_{z_{2}}\right)\left(\partial_{z_{1}} \cdot \partial_{z_{1}}\right)\right] .
\end{aligned}
$$

Moreover, these operators automatically satisfy

$$
\delta_{a b} D_{i}^{a} D_{i}^{b}=0, \quad\left[D_{i}^{a}, D_{i}^{b}\right]=0, \quad\left[D_{1}^{a}, D_{2}^{b}\right]=0,
$$

for $i \in\{1,2\}$. The general normalization factor appearing in (A.4) is fixed asking for the idempotence of the projector,

$$
\mathcal{N}_{l_{1}, l_{2}}=\frac{(-1)^{l_{2}-l_{1}} 2^{l_{2}}\left(l_{1}-l_{2}+1\right)}{\Gamma\left(l_{1}+2\right) \Gamma\left(l_{2}+1\right)\left(\frac{d}{2}-1\right)_{l_{1}}\left(\frac{d}{2}-2\right)_{l_{2}}(d-3)_{l_{1}+l_{2}}} .
$$

As an example we construct the projector into the representations $\square 11 \cdots$,

$$
\pi_{\left(l_{1}, 1\right)}^{\left[a_{1} a_{2}\right] a_{3} \ldots a_{l_{1}+1},\left[b_{1} b_{2}\right] b_{3} \ldots b_{l_{1}+1}}=\mathcal{N}_{l_{1}, 1} D_{1}^{\left[a_{1}\right.} D_{2}^{\left.a_{2}\right]} D_{1}^{a_{3}} \cdots D_{1}^{a_{l_{1}+1}} z_{1}^{\left[b_{1}\right.} z_{2}^{\left.b_{2}\right]} z_{1}^{b_{3}} \cdots z_{1}^{b_{l_{1}+1}} .
$$

The projector that is generated in this way is in the antisymmetric representation. It is related to the other expressions derived in the main text by the contraction

$$
\begin{aligned}
\pi_{\lambda}\left(z_{1}, z_{2}, \bar{z}_{1}, \bar{z}_{2}\right)= & n_{\lambda} z_{1}^{a_{1}} z_{2}^{a_{2}} \ldots z_{1}^{a_{2 l_{2}-1}} z_{2}^{a_{2 l_{2}}} z_{1}^{a_{2 l_{2}+1}} \ldots z_{1}^{a_{l_{1}+l_{2}}} \\
& \pi_{\lambda}^{\left[a_{1} a_{2}\right] \ldots\left[a_{2 l_{2}-1} a_{2 l_{2}}\right] a_{2 l_{2}+1} \ldots a_{l_{1}+l_{2}},\left[b_{1} b_{2}\right] \ldots\left[b_{2 l_{2}-1} b_{2 l_{2}}\right] b_{2 l_{2}+1} \ldots b_{l_{1}+l_{2}}} \\
& \bar{z}_{1}^{b_{1}} \bar{z}_{2}^{b_{2}} \ldots \bar{z}_{1}^{b_{2 l_{2}-1}} \bar{z}_{2}^{b_{2 l_{2}}} \bar{z}_{1}^{b_{2 l_{2}+1}} \ldots \bar{z}_{1}^{b_{l_{1}+l_{2}}}
\end{aligned}
$$

\footnotetext{
${ }^{6}$ There exist higher order differential operators that satisfy the same requirements. The ones that we found are the lowest order ones.
} 
where the factor $n_{\lambda}$ appears due to the change from the antisymmetric to the symmetric representation and is defined in (2.60).

It is in principle possible to generalize this method in order to study Young diagrams with more than two rows. However this requires to find a new set of differential operators, which we expect to be lengthy and therefore not very efficient.

\section{B Relating different projectors}

This section introduces a useful relation between similar projectors. The main observation is that a contraction between corresponding indices on the left and right of a projector leads to an object that is still traceless and has mixed-symmetry. Hence it should be proportional to another projector, e.g.

$$
\delta_{a_{1} b_{1}} \pi_{\left(l_{1}, l_{2}, l_{3}\right)}^{\left(a_{1} \ldots a_{l_{1}}\right) \ldots\left(\ldots a_{l_{1}+l_{2}+l_{3}}\right),\left(b_{1} \ldots b_{l_{1}}\right) \ldots\left(\ldots b_{l_{1}+l_{2}+l_{3}}\right)} \propto \pi_{\left(l_{1}-1, l_{2}, l_{3}\right)}^{\left(a_{2} \ldots a_{l_{1}}\right) \ldots\left(\ldots a_{l_{1}+l_{2}+l_{3}}\right),\left(b_{2} \ldots b_{l_{1}}\right) \ldots\left(\ldots b_{l_{1}+l_{2}+l_{3}}\right)} .
$$

Using auxiliary vectors $\left\{z_{i}, \bar{z}_{i}\right\}(i=1,2,3)$, this equation can be written as

$$
\frac{1}{\left(l_{1}\right)^{2}}\left(\frac{\partial}{\partial z_{1}} \cdot \frac{\partial}{\partial \bar{z}_{1}}\right) \pi_{\left(l_{1}, l_{2}, l_{3}\right)}\left(\left\{z_{i}, \bar{z}_{i}\right\}\right) \propto \pi_{\left(l_{1}-1, l_{2}, l_{3}\right)}\left(\left\{z_{i}, \bar{z}_{i}\right\}\right) .
$$

The proportionality factor can be found by using that the full trace of a projector is given by the dimension $d_{\lambda}$ of the $\mathrm{SO}(d)$ irrep,

$$
\frac{1}{\left(l_{1} ! l_{2} ! l_{3} !\right)^{2}}\left(\frac{\partial}{\partial z_{1}} \cdot \frac{\partial}{\partial \bar{z}_{1}}\right)^{l_{1}}\left(\frac{\partial}{\partial z_{2}} \cdot \frac{\partial}{\partial \bar{z}_{2}}\right)^{l_{2}}\left(\frac{\partial}{\partial z_{3}} \cdot \frac{\partial}{\partial \bar{z}_{3}}\right)^{l_{3}} \pi_{\left(l_{1}, l_{2}, l_{3}\right)}\left(\left\{z_{i}, \bar{z}_{i}\right\}\right)=d_{\left(l_{1}, l_{2}, l_{3}\right)}
$$

which is given by [53]

$$
d_{\lambda}=\frac{1}{H(\lambda)} \prod_{i=1}^{h_{1}} \prod_{j=1}^{\min \left(i, l_{i}\right)}\left(d+l_{i}+l_{j}-i-j\right) \prod_{j=2}^{l_{1}} \prod_{i=1}^{\min \left(j-1, h_{j}\right)}\left(d-h_{i}-h_{j}+i+j-2\right),
$$

where $H(\lambda)$ was defined in (2.60). Since both sides in (B.2) can be reduced to the corresponding dimensions by doing full contractions, the missing constant is given by the ratios of dimensions

$$
\frac{1}{\left(l_{1}\right)^{2}}\left(\frac{\partial}{\partial z_{1}} \cdot \frac{\partial}{\partial \bar{z}_{1}}\right) \pi_{\left(l_{1}, l_{2}, l_{3}\right)}\left(\left\{z_{i}, \bar{z}_{i}\right\}\right)=\frac{d_{\left(l_{1}, l_{2}, l_{3}\right)}}{d_{\left(l_{1}-1, l_{2}, l_{3}\right)}} \pi_{\left(l_{1}-1, l_{2}, l_{3}\right)}\left(\left\{z_{i}, \bar{z}_{i}\right\}\right) .
$$

Of course the same relation holds also for the other rows in the Young diagram, e.g.

$$
\frac{1}{\left(l_{2}\right)^{2}}\left(\frac{\partial}{\partial z_{2}} \cdot \frac{\partial}{\partial \bar{z}_{2}}\right) \pi_{\left(l_{1}, l_{2}, l_{3}\right)}\left(\left\{z_{i}, \bar{z}_{i}\right\}\right)=\frac{d_{\left(l_{1}, l_{2}, l_{3}\right)}}{d_{\left(l_{1}, l_{2}-1, l_{3}\right)}} \pi_{\left(l_{1}, l_{2}-1, l_{3}\right)}\left(\left\{z_{i}, \bar{z}_{i}\right\}\right) .
$$

\section{More projectors}

In this appendix we state the remaining projectors to irreducible representations of $\mathrm{SO}(d)$ that can appear in a three-point function with two stress-tensors. 


\section{C.1 Projectors to the irreps $\left(l_{1}, 3\right)$}

For Young diagrams of shape $\amalg^{1 \cdots}$ the possible combinations of the proposed building blocks are

$$
\begin{aligned}
& Q_{1}=H\left(z_{2}, \bar{z}_{2}\right)^{3}, \\
& Q_{2}=H\left(z_{2}, \bar{z}_{2}\right)^{2} V\left(z_{2}\right) \bar{V}\left(\bar{z}_{2}\right), \\
& Q_{3}=H\left(z_{2}, \bar{z}_{2}\right) V\left(z_{2}\right)^{2} \bar{V}\left(\bar{z}_{2}\right)^{2}, \\
& Q_{4}=V\left(z_{2}\right)^{3} \bar{V}\left(\bar{z}_{2}\right)^{3}, \\
& Q_{5}=H\left(z_{2}, \bar{z}_{2}\right) T\left(z_{2}, z_{2}\right) \bar{T}\left(\bar{z}_{2}, \bar{z}_{2}\right), \\
& Q_{6}=V\left(z_{2}\right) \bar{V}\left(\bar{z}_{2}\right) T\left(z_{2}, z_{2}\right) \bar{T}\left(\bar{z}_{2}, \bar{z}_{2}\right), \\
& Q_{7}=H\left(z_{2}, \bar{z}_{2}\right)\left(T\left(z_{2}, z_{2}\right) \bar{V}\left(\bar{z}_{2}\right)^{2}+V\left(z_{2}\right)^{2} \bar{T}\left(\bar{z}_{2}, \bar{z}_{2}\right)\right), \\
& Q_{8}=V\left(z_{2}\right) \bar{V}\left(\bar{z}_{2}\right)\left(T\left(z_{2}, z_{2}\right) \bar{V}\left(\bar{z}_{2}\right)^{2}+V\left(z_{2}\right)^{2} \bar{T}\left(\bar{z}_{2}, \bar{z}_{2}\right)\right) .
\end{aligned}
$$

Imposing the tracelessness conditions (2.20) one finds that the functions $f_{i}(t)$ that appear in the projector $(2.14)$ can then be written as

$$
f_{i}(t)=-\frac{\hat{f}_{i}(t)}{d}, \quad \forall i=1, \ldots, 8
$$

with

$$
\begin{aligned}
\hat{f}_{1}(t)= & -d(1+d)(2+d) t\left(-3+d t^{2}\right) \mathcal{C}_{l_{1}}^{(3)}(t)-3(1+d)(2+d)\left(t^{2}-1\right)\left(d t^{2}-1\right) \mathcal{C}_{l_{1}}^{(4)}(t) \\
& -3 d(2+d) t\left(t^{2}-1\right)^{2} \mathcal{C}_{l_{1}}^{(5)}(t)-d\left(t^{2}-1\right)^{3} \mathcal{C}_{l_{1}}^{(6)}(t) \\
\hat{f}_{2}(t)= & 6 d(1+d)(2+d) t \mathcal{C}_{l_{1}}^{(3)}(t)+3(1+d)(2+d)\left(-3+(4+d) t^{2}\right) \mathcal{C}_{l_{1}}^{(4)}(t) \\
& +6(1+d)(2+d) t\left(t^{2}-1\right) \mathcal{C}_{l_{1}}^{(5)}(t)+3 d\left(t^{2}-1\right)^{2} \mathcal{C}_{l_{1}}^{(6)}(t) \\
\hat{f}_{3}(t)= & 3 d(1+d)(2+d) t \mathcal{C}_{l_{1}}^{(3)}(t)+9(1+d)(2+d)\left(t^{2}-1\right) \mathcal{C}_{l_{1}}^{(4)}(t) \\
& -3(2+d) t\left(4+d-3 t^{2}\right) \mathcal{C}_{l_{1}}^{(5)}(t)-3\left(d-t^{2}\right)\left(t^{2}-1\right) \mathcal{C}_{l_{1}}^{(6)}(t) \\
\hat{f}_{4}(t)= & -3(1+d)(2+d) \mathcal{C}_{l_{1}}^{(4)}(t)-6(2+d) t \mathcal{C}_{l_{1}}^{(5)}(t)+\left(d-3 t^{2}\right) \mathcal{C}_{l_{1}}^{(6)}(t), \\
\hat{f}_{5}(t)= & 3 d(2+d) t\left(-4+(1+d) t^{2}\right) \mathcal{C}_{l_{1}}^{(3)}(t)+3(2+d)\left(t^{2}-1\right)(-4) \\
& +9(2+d) t\left(t^{2}-1\right)^{2} \mathcal{C}_{l_{1}}^{(5)}(t)+3\left(t^{2}-1\right)^{3} \mathcal{C}_{l_{1}}^{(6)}(t) \\
\hat{f}_{6}(t)= & -3(2+d)\left(-4+(1+d) t^{2}\right) \mathcal{C}_{l_{1}}^{(4)}(t)-6(2+d) t\left(t^{2}-1\right) \mathcal{C}_{l_{1}}^{(5)}(t)-3\left(t^{2}-1\right)^{2} \mathcal{C}_{l_{1}}^{(6)}(t), \\
\hat{f}_{7}(t)= & 3 d(1+d)(2+d) t^{2} \mathcal{C}_{l_{1}}^{(3)}(t)+3(1+d)(2+d) t\left(-1+3 t^{2}\right) \mathcal{C}_{l_{1}}^{(4)}(t) \\
& +9(2+d) t^{2}\left(t^{2}-1\right) \mathcal{C}_{l_{1}}^{(5)}(t)+3 t\left(t^{2}-1\right)^{2} \mathcal{C}_{l_{1}}^{(6)}(t), \\
\hat{f}_{8}(t)= & -3(1+d)(2+d) t \mathcal{C}_{l_{1}}^{(4)}(t)-6(2+d) t^{2} \mathcal{C}_{l_{1}}^{(5)}(t)-3 t\left(t^{2}-1\right) \mathcal{C}_{l_{1}}^{(6)}(t) .
\end{aligned}
$$




\section{C.2 Projectors to the irreps $\left(l_{1}, 4\right)$}

For Young diagrams of shape $\# H^{--\square}$ the required building blocks are

$$
\begin{aligned}
Q_{1} & =H\left(z_{2}, \bar{z}_{2}\right)^{4} \\
Q_{2} & =H\left(z_{2}, \bar{z}_{2}\right)^{3} V\left(z_{2}\right) \bar{V}\left(\bar{z}_{2}\right), \\
Q_{3} & =H\left(z_{2}, \bar{z}_{2}\right)^{2} V\left(z_{2}\right)^{2} \bar{V}\left(\bar{z}_{2}\right)^{2}, \\
Q_{4} & =H\left(z_{2}, \bar{z}_{2}\right) V\left(z_{2}\right)^{3} \bar{V}\left(\bar{z}_{2}\right)^{3} \\
Q_{5} & =V\left(z_{2}\right)^{4} \bar{V}\left(\bar{z}_{2}\right)^{4} \\
Q_{6} & =H\left(z_{2}, \bar{z}_{2}\right)^{2} T\left(z_{2}, z_{2}\right) \bar{T}\left(\bar{z}_{2}, \bar{z}_{2}\right), \\
Q_{7} & =H\left(z_{2}, \bar{z}_{2}\right) V\left(z_{2}\right) \bar{V}\left(\bar{z}_{2}\right) T\left(z_{2}, z_{2}\right) \bar{T}\left(\bar{z}_{2}, \bar{z}_{2}\right), \\
Q_{8} & =V\left(z_{2}\right)^{2} \bar{V}\left(\bar{z}_{2}\right)^{2} T\left(z_{2}, z_{2}\right) \bar{T}\left(\bar{z}_{2}, \bar{z}_{2}\right), \\
Q_{9} & =T\left(z_{2}, z_{2}\right)^{2} \bar{T}\left(\bar{z}_{2}, \bar{z}_{2}\right)^{2}, \\
Q_{10} & =H\left(z_{2}, \bar{z}_{2}\right)^{2}\left(T\left(z_{2}, z_{2}\right) \bar{V}\left(\bar{z}_{2}\right)^{2}+V\left(z_{2}\right)^{2} \bar{T}\left(\bar{z}_{2}, \bar{z}_{2}\right)\right), \\
Q_{11} & =H\left(z_{2}, \bar{z}_{2}\right) V\left(z_{2}\right) \bar{V}\left(\bar{z}_{2}\right)\left(T\left(z_{2}, z_{2}\right) \bar{V}\left(\bar{z}_{2}\right)^{2}+V\left(z_{2}\right)^{2} \bar{T}\left(\bar{z}_{2}, \bar{z}_{2}\right)\right), \\
Q_{12} & =V\left(z_{2}\right)^{2} \bar{V}\left(\bar{z}_{2}\right)^{2}\left(T\left(z_{2}, z_{2}\right) \bar{V}\left(\bar{z}_{2}\right)^{2}+V\left(z_{2}\right)^{2} \bar{T}\left(\bar{z}_{2}, \bar{z}_{2}\right)\right), \\
Q_{13} & =T\left(z_{2}, z_{2}\right)^{2} \bar{V}\left(\bar{z}_{2}\right)^{4}+V\left(z_{2}\right)^{4} \bar{T}\left(\bar{z}_{2}, \bar{z}_{2}\right)^{2}, \\
Q_{14} & =T\left(z_{2}, z_{2}\right) \bar{T}\left(\bar{z}_{2}, \bar{z}_{2}\right)\left(T\left(z_{2}, z_{2}\right) \bar{V}\left(\bar{z}_{2}\right)^{2}+V\left(z_{2}\right)^{2} \bar{T}\left(\bar{z}_{2}, \bar{z}_{2}\right)\right) .
\end{aligned}
$$

In this case the functions $f_{i}(t)$ that appear in the projector $(2.14)$ can then be written as

$$
f_{i}(t)=-\frac{\hat{f}_{i}(t)}{d(d+2)}, \quad \forall i=1, \ldots, 14,
$$

with

$$
\begin{aligned}
\hat{f}_{1}(t)= & (d+1)_{4}\left(6 d t^{2}-3-d(2+d) t^{4}\right) \mathcal{C}_{l_{1}}^{(4)}(t)-4 d(d+2)_{3} t\left(t^{2}-1\right)\left((2+d) t^{2}-3\right) \mathcal{C}_{l_{1}}^{(5)}(t) \\
& -6 d(d+3)_{2}\left(t^{2}-1\right)^{2}\left(-1+(2+d) t^{2}\right) \mathcal{C}_{l_{1}}^{(6)}(t)-4 d(2+d)(4+d) t\left(t^{2}-1\right)^{3} \mathcal{C}_{l_{1}}^{(7)}(t) \\
& -d(2+d)\left(t^{2}-1\right)^{4} \mathcal{C}_{l_{1}}^{(8)}(t), \\
\hat{f}_{2}(t)= & 12(d+1)_{4}\left(d t^{2}-1\right) \mathcal{C}_{l_{1}}^{(4)}(t)+4(d+2)_{3} t\left(-3-9 d+d(11+d) t^{2}\right) \mathcal{C}_{l_{1}}^{(5)}(t) \\
& +12 d(d+3)_{2}\left(t^{2}-1\right)\left(-2+(5+d) t^{2}\right) \mathcal{C}_{l_{1}}^{(6)}(t)+12 d(d+3)_{2} t\left(t^{2}-1\right)^{2} \mathcal{C}_{l_{1}}^{(7)}(t) \\
& +4 d(2+d)\left(t^{2}-1\right)^{3} \mathcal{C}_{l_{1}}^{(8)}(t), \\
\hat{f}_{3}(t)= & 6(d+1)_{4}\left(-3+d t^{2}\right) \mathcal{C}_{l_{1}}^{(4)}(t)+12(d+2)_{3} t\left(-3+d\left(-3+2 t^{2}\right)\right) \mathcal{C}_{l_{1}}^{(5)}(t) \\
& -6(d+3)_{2}\left(-6 d+(3+d(16+d)) t^{2}-6 d t^{4}\right) \mathcal{C}_{l_{1}}^{(6)}(t) \\
& -12 d(4+d) t\left(5+d-2 t^{2}\right)\left(t^{2}-1\right) \mathcal{C}_{l_{1}}^{(7)}(t)-6 d\left(2+d-t^{2}\right)\left(t^{2}-1\right)^{2} \mathcal{C}_{l_{1}}^{(8)}(t), \\
\hat{f}_{4}(t)= & -12(d+1)_{4} \mathcal{C}_{l_{1}}^{(4)}(t)-12(2+d)(3+d)^{2}(4+d) t \mathcal{C}_{l_{1}}^{(5)}(t) \\
& -12(d+3)_{2}\left(-2 d+3(1+d) t^{2}\right) \mathcal{C}_{l_{1}}^{(6)}(t)+4(4+d) t\left(d(11+d)-3(1+3 d) t^{2}\right) \mathcal{C}_{l_{1}}^{(7)}(t) \\
& +4 d\left(2+d-3 t^{2}\right)\left(t^{2}-1\right) \mathcal{C}_{l_{1}}^{(8)}(t),
\end{aligned}
$$




$$
\begin{aligned}
& \hat{f}_{5}(t)=-3(d+1)_{4} \mathcal{C}_{l_{1}}^{(4)}(t)-12(d+2)_{3} \mathcal{C}_{l_{1}}^{(5)}(t)+6(d+3)_{2}\left(d-3 t^{2}\right) \mathcal{C}_{l_{1}}^{(6)}(t) \\
& +12(4+d) t\left(d-t^{2}\right) \mathcal{C}_{l_{1}}^{(7)}(t)+\left(-d(2+d)+6 d t^{2}-3 t^{4}\right) \mathcal{C}_{l_{1}}^{(8)}(t), \\
& \hat{f}_{6}(t)=6(1+d)(2+d)(4+d)\left(4-(1+7 d) t^{2}+d(3+d) t^{4}\right) \mathcal{C}_{l_{1}}^{(4)}(t) \\
& +12(2+d)(4+d) t\left(t^{2}-1\right)\left(-1-7 d+2 d(3+d) t^{2}\right) \mathcal{C}_{l_{1}}^{(5)}(t) \\
& +6(4+d)\left(t^{2}-1\right)^{2}\left(-1-7 d+6 d(3+d) t^{2}\right) \mathcal{C}_{l_{1}}^{(6)}(t)+24 d(4+d) t\left(t^{2}-1\right)^{3} \mathcal{C}_{l_{1}}^{(7)}(t) \\
& +6 d\left(t^{2}-1\right)^{4} \mathcal{C}_{l_{1}}^{(8)}(t) \\
& \hat{f}_{7}(t)=-12(1+d)(2+d)(4+d)\left(-4+(3+d) t^{2}\right) \mathcal{C}_{l_{1}}^{(4)}(t) \\
& -12(2+d)(4+d) t\left(-8(1+d)+(3+d)^{2} t^{2}\right) \mathcal{C}_{l_{1}}^{(5)}(t) \\
& -12(4+d)\left(t^{2}-1\right)\left(-1-7 d+3(1+d)(3+d) t^{2}\right) \mathcal{C}_{l_{1}}^{(6)}(t) \\
& -12(4+d)(1+3 d) t\left(t^{2}-1\right)^{2} \mathcal{C}_{l_{1}}^{(7)}(t)-12 d\left(t^{2}-1\right)^{3} \mathcal{C}_{l_{1}}^{(8)}(t), \\
& \hat{f}_{8}(t)=-12(1+d)(2+d)(4+d)\left(-2+(3+d) t^{2}\right) \mathcal{C}_{l_{1}}^{(4)}(t) \\
& -12(2+d)(4+d) t\left(-7-d+4(3+d) t^{2}\right) \mathcal{C}_{l_{1}}^{(5)}(t) \\
& +6(4+d)\left(-1-7 d+(4+d)(7+d) t^{2}-12(3+d) t^{4}\right) \mathcal{C}_{l_{1}}^{(6)}(t) \\
& +12(4+d) t\left(1+d-4 t^{2}\right)\left(t^{2}-1\right) \mathcal{C}_{l_{1}}^{(7)}(t)+6\left(d-2 t^{2}\right)\left(t^{2}-1\right)^{2} \mathcal{C}_{l_{1}}^{(8)}(t), \\
& \hat{f}_{9}(t)=-3(2+d)(4+d)\left(8+(1+d) t^{2}\left(-8+(3+d) t^{2}\right)\right) \mathcal{C}_{l_{1}}^{(4)}(t) \\
& -12(2+d)(4+d) t\left(t^{2}-1\right)\left(-4+(3+d) t^{2}\right) \mathcal{C}_{l_{1}}^{(5)}(t) \\
& -6(4+d)\left(t^{2}-1\right)^{2}\left(-4+3(3+d) t^{2}\right) \mathcal{C}_{l_{1}}^{(6)}(t)-12(4+d) t\left(t^{2}-1\right)^{3} \mathcal{C}_{l_{1}}^{(7)}(t) \\
& -3\left(t^{2}-1\right)^{4} \mathcal{C}_{l_{1}}^{(8)}(t), \\
& \hat{f}_{10}(t)=6(d+1)_{4} t\left(d t^{2}-1\right) \mathcal{C}_{l_{1}}^{(4)}(t)+12(d+2)_{3} t^{2}\left(-1+d\left(-1+2 t^{2}\right)\right) \mathcal{C}_{l_{1}}^{(5)}(t) \\
& +6(d+3){ }_{2} t\left(t^{2}-1\right)\left(-1+d\left(-1+6 t^{2}\right)\right) \mathcal{C}_{l_{1}}^{(6)}(t)+24 d(4+d) t^{2}\left(t^{2}-1\right)^{2} \mathcal{C}_{l_{1}}^{(7)}(t) \\
& +6 d t\left(t^{2}-1\right)^{3} \mathcal{C}_{l_{1}}^{(8)}(t), \\
& \hat{f}_{11}(t)=-12(d+1)_{4} t \mathcal{C}_{l_{1}}^{(4)}(t)-12(2+d)(3+d)^{2}(4+d) t^{2} \mathcal{C}_{l_{1}}^{(5)}(t) \\
& -12(1+d)(d+3)_{2} t\left(-1+3 t^{2}\right) \mathcal{C}_{l_{1}}^{(6)}(t)-12(4+d)(1+3 d) t^{2}\left(t^{2}-1\right) \mathcal{C}_{l_{1}}^{(7)}(t) \\
& -12 d t\left(t^{2}-1\right)^{2} \mathcal{C}_{l_{1}}^{(8)}(t), \\
& \hat{f}_{12}(t)=-6(d+1)_{4} t \mathcal{C}_{l_{1}}^{(4)}(t)-24(d+2)_{3} t^{2} \mathcal{C}_{l_{1}}^{(5)}(t)+6(d+3)_{2} t\left(1+d-6 t^{2}\right) \mathcal{C}_{l_{1}}^{(6)}(t) \\
& +12(4+d) t^{2}\left(1+d-2 t^{2}\right) \mathcal{C}_{l_{1}}^{(7)}(t)+6 t\left(d-t^{2}\right)\left(t^{2}-1\right) \mathcal{C}_{l_{1}}^{(8)}(t), \\
& \hat{f}_{13}(t)=-3(d+1)_{4} t^{2} \mathcal{C}_{l_{1}}^{(4)}(t)-12(d+2)_{3} t^{3} \mathcal{C}_{l_{1}}^{(5)}(t)-6(d+3)_{2} t^{2}\left(-1+3 t^{2}\right) \mathcal{C}_{l_{1}}^{(6)}(t) \\
& -12(4+d) t^{3}\left(t^{2}-1\right) \mathcal{C}_{l_{1}}^{(7)}(t)-3 t^{2}\left(t^{2}-1\right)^{2} \mathcal{C}_{l_{1}}^{(8)}(t),
\end{aligned}
$$




$$
\begin{aligned}
\hat{f}_{14}(t)= & -6(1+d)(2+d)(4+d) t\left(-4+(3+d) t^{2}\right) \mathcal{C}_{l_{1}}^{(4)}(t) \\
& -12(2+d)(4+d) t^{2}\left(-7-d+2(3+d) t^{2}\right) \mathcal{C}_{l_{1}}^{(5)}(t) \\
& -6(4+d) t\left(t^{2}-1\right)\left(-7-d+6(3+d) t^{2}\right) \mathcal{C}_{l_{1}}^{(6)}(t) \\
& -24(4+d) t^{2}\left(t^{2}-1\right)^{2} \mathcal{C}_{l_{1}}^{(7)}(t)-6 t\left(t^{2}-1\right)^{3} \mathcal{C}_{l_{1}}^{(8)}(t) .
\end{aligned}
$$

\section{C.3 Projectors to the irreps $\left(l_{1}, 3,1\right)$}

To construct the tensor structures for projectors to irreps reducible representation for the shape $\square$, given by

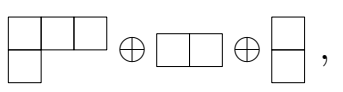

with the building blocks
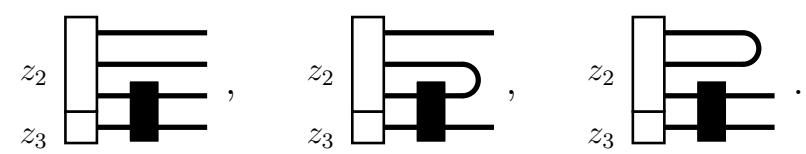

Note that the second expression here is not symmetric in its indices. However, the third expression is antisymmetric and the second one has a symmetric part, so this will suffice as a basis. From the tensor product

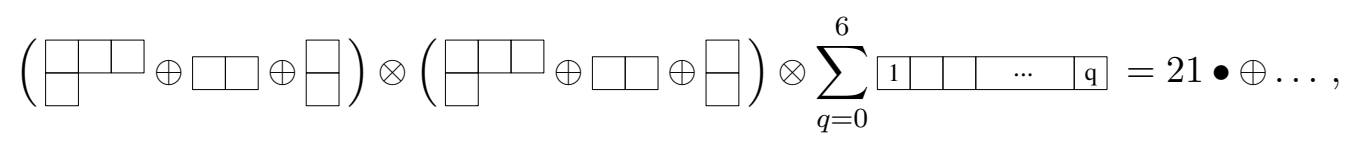

we conclude that in this case there will be 21 allowed structures, which can be identified with different terms in the tensor product

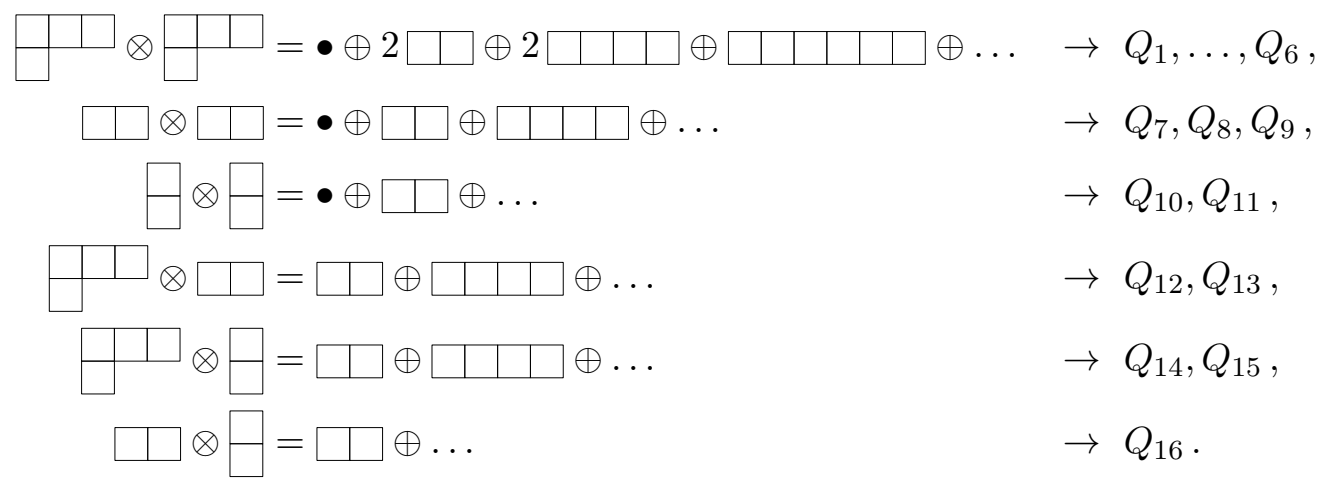


Using the birdtrack notation (2.23), each $Q_{i}$ has the form
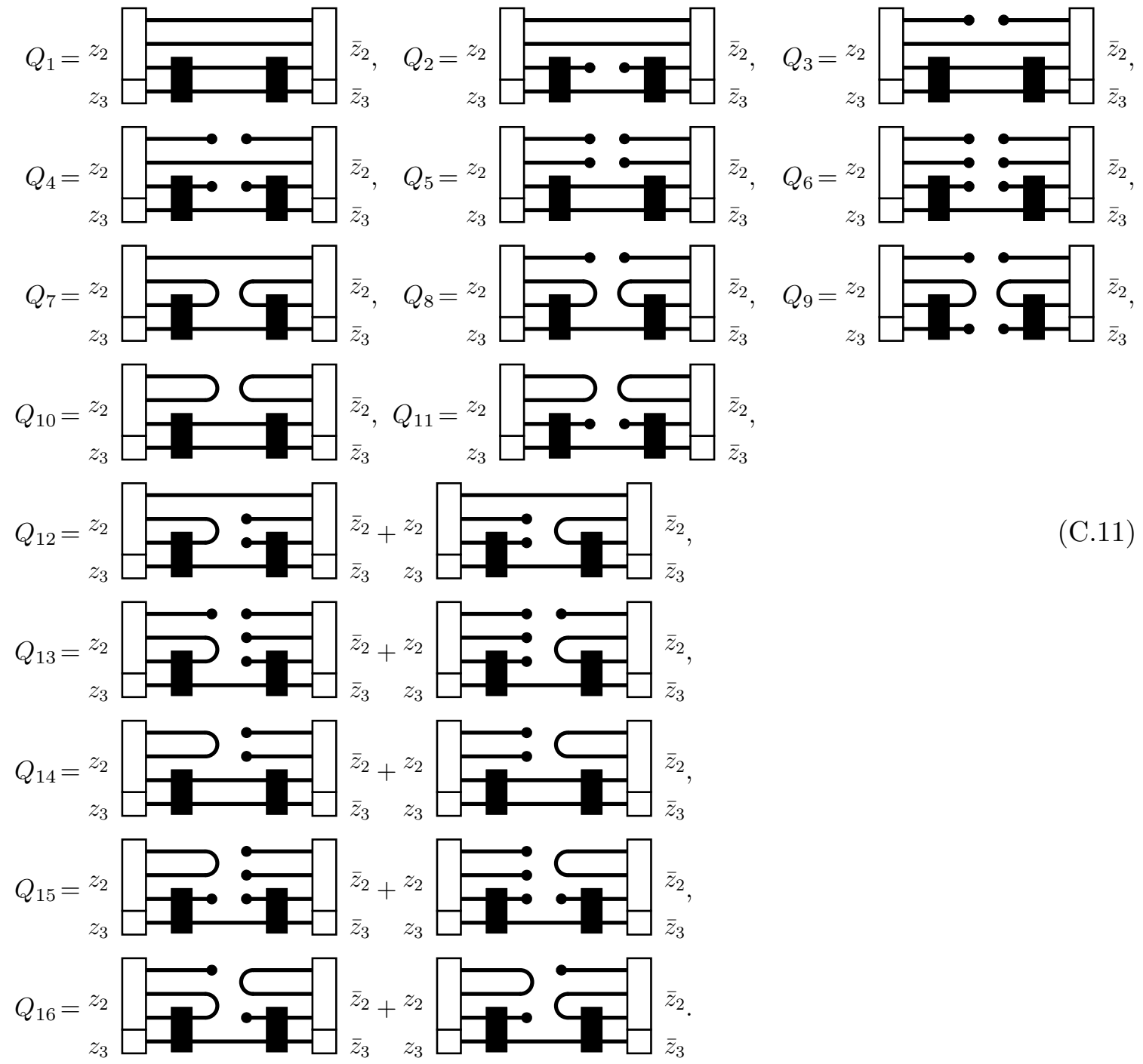

The functions $f_{i}(t)$ can now be written as

$$
f_{i}(t)=-\frac{\hat{f}_{i}(t)}{(d-2) d}, \quad \forall i=1, \ldots, 16,
$$

with

$$
\begin{aligned}
\hat{f}_{1}(t)= & -(d-1)(1+d)_{2} t\left(4-3 d+(d-2) d t^{2}\right) \mathcal{C}_{l_{1}}^{(3)}(t) \\
& -(2+d)\left(t^{2}-1\right)\left(4-3(d-1) d+(d-2) d(1+3 d) t^{2}\right) \mathcal{C}_{l_{1}}^{(4)}(t) \\
& -(d-2) d(5+3 d) t\left(t^{2}-1\right)^{2} \mathcal{C}_{l_{1}}^{(5)}(t)-(d-2) d\left(t^{2}-1\right)^{3} \mathcal{C}_{l_{1}}^{(6)}(t), \\
\hat{f}_{2}(t)= & 4(d-1)_{4} t \mathcal{C}_{l_{1}}^{(3)}(t)+2(2+d)\left(4-3(d-1) d+d(-10+d(5+d)) t^{2}\right) \mathcal{C}_{l_{1}}^{(4)}(t) \\
& +4 d(-7+d(2+d)) t\left(t^{2}-1\right) \mathcal{C}_{l_{1}}^{(5)}(t)+2(d-2) d\left(t^{2}-1\right)^{2} \mathcal{C}_{l_{1}}^{(6)}(t), \\
\hat{f}_{3}(t)= & 4\left(4-5 d^{2}+d^{4}\right) t \mathcal{C}_{l_{1}}^{(3)}(t)+2(2+d)\left(4-4 t^{2}+(d-1) d\left(-3+(2+d) t^{2}\right)\right) \mathcal{C}_{l_{1}}^{(4)}(t) \\
& +4\left(-4-d+d^{3}\right) t\left(t^{2}-1\right) \mathcal{C}_{l_{1}}^{(5)}(t)+2(d-2) d\left(t^{2}-1\right)^{2} \mathcal{C}_{l_{1}}^{(6)}(t),
\end{aligned}
$$




$$
\begin{aligned}
& \hat{f}_{4}(t)=4(d-1){ }_{4} t \mathcal{C}_{l_{1}}^{(3)}(t)+4(2+d)\left(4+d\left(3+t^{2}+3 d\left(t^{2}-1\right)\right)\right) \mathcal{C}_{l_{1}}^{(4)}(t) \\
& +4 t\left(8+d\left(6+5 t^{2}-d\left(5+d-3 t^{2}\right)\right)\right) \mathcal{C}_{l_{1}}^{(5)}(t)+4 d\left(-2+d+t^{2}-d t^{2}+t^{4}\right) \mathcal{C}_{l_{1}}^{(6)}(t), \\
& \hat{f}_{5}(t)=(d-4)(d-1)(1+d)_{2} t \mathcal{C}_{l_{1}}^{(3)}(t)+(2+d)\left(4+3 d-3 d^{2}+(d-4)(1+3 d) t^{2}\right) \mathcal{C}_{l_{1}}^{(4)}(t) \\
& +t\left(16-d\left(6+d+d^{2}\right)+(d-4)(5+3 d) t^{2}\right) \mathcal{C}_{l_{1}}^{(5)}(t) \\
& +\left(t^{2}-1\right)\left(-(d-2) d+(d-4) t^{2}\right) \mathcal{C}_{l_{1}}^{(6)}(t), \\
& \hat{f}_{6}(t)=2(2+d)\left(4+3 d-3 d^{2}\right) \mathcal{C}_{l_{1}}^{(4)}(t)-4\left(3 d^{2}+d-8\right) t \mathcal{C}_{l_{1}}^{(5)}(t) \\
& +\left(2(d-2) d+2(4-3 d) t^{2}\right) \mathcal{C}_{l_{1}}^{(6)}(t), \\
& \hat{f}_{7}(t)=4(d-1)(1+d)_{2} t\left(-4+d t^{2}\right) \mathcal{C}_{l_{1}}^{(3)}(t)+4 d(2+d)\left(t^{2}-1\right)\left(-4+(1+3 d) t^{2}\right) \mathcal{C}_{l_{1}}^{(4)}(t) \\
& +4 d(5+3 d) t\left(t^{2}-1\right)^{2} \mathcal{C}_{l_{1}}^{(5)}(t)+4 d\left(t^{2}-1\right)^{3} \mathcal{C}_{l_{1}}^{(6)}(t), \\
& \hat{f}_{8}(t)=-16(d-1)(1+d)_{2} t \mathcal{C}_{l_{1}}^{(3)}(t)+8(2+d)\left(4 d-(-2+d(5+d)) t^{2}\right) \mathcal{C}_{l_{1}}^{(4)}(t) \\
& -16 d(3+d) t\left(t^{2}-1\right) \mathcal{C}_{l_{1}}^{(5)}(t)-8 d\left(t^{2}-1\right)^{2} \mathcal{C}_{l_{1}}^{(6)}(t), \\
& \hat{f}_{9}(t)=16 d(2+d) \mathcal{C}_{l_{1}}^{(4)}(t)+4 d(7+d) t \mathcal{C}_{l_{1}}^{(5)}(t)+4 d\left(t^{2}-1\right) \mathcal{C}_{l_{1}}^{(6)}(t), \\
& \hat{f}_{10}(t)=(d-4)\left(d^{2}+d-2\right) t\left((1+d) t^{2}-4\right) \mathcal{C}_{l_{1}}^{(3)}(t)+(d-4)(2+d)\left((1+3 d) t^{2}-4\right) \\
& \times\left(t^{2}-1\right) \mathcal{C}_{l_{1}}^{(4)}(t)+(d-4)(5+3 d) t\left(t^{2}-1\right)^{2} \mathcal{C}_{l_{1}}^{(5)}(t)+(d-4)\left(t^{2}-1\right)^{3} \mathcal{C}_{l_{1}}^{(6)}(t), \\
& \hat{f}_{11}(t)=-16(d-1)(1+d){ }_{2} t \mathcal{C}_{l_{1}}^{(3)}(t)-2(2+d)\left(16-12 d+(-28+3 d(7+d)) t^{2}\right) \mathcal{C}_{l_{1}}^{(4)}(t) \\
& -4(-16+3 d(3+d)) t\left(t^{2}-1\right) \mathcal{C}_{l_{1}}^{(5)}(t)-2(3 d-4)\left(t^{2}-1\right)^{2} \mathcal{C}_{l_{1}}^{(6)}(t), \\
& \hat{f}_{12}(t)=4(d-1)_{4} t^{2} \mathcal{C}_{l_{1}}^{(3)}(t)+4(2+d) t\left(-4+d-d^{2}+d(1+3 d) t^{2}\right) \mathcal{C}_{l_{1}}^{(4)}(t) \\
& +4 d(5+3 d) t^{2}\left(t^{2}-1\right) \mathcal{C}_{l_{1}}^{(5)}(t)+4 d t\left(t^{2}-1\right)^{2} \mathcal{C}_{l_{1}}^{(6)}(t), \\
& \hat{f}_{13}(t)=-4\left(8+d\left(2+d+d^{2}\right)\right) t \mathcal{C}_{l_{1}}^{(4)}(t)-8\left(2+d+d^{2}\right) t^{2} \mathcal{C}_{l_{1}}^{(5)}(t)-4 d t\left(t^{2}-1\right) \mathcal{C}_{l_{1}}^{(6)}(t), \\
& \hat{f}_{14}(t)=(d-4)(d-1)(1+d){ }_{2} t^{2} \mathcal{C}_{l_{1}}^{(3)}(t)+(d-4)(2+d) t\left(-3+t^{2}+d\left(-1+3 t^{2}\right)\right) \mathcal{C}_{l_{1}}^{(4)}(t) \\
& +(d-4)(5+3 d) t^{2}\left(t^{2}-1\right) \mathcal{C}_{l_{1}}^{(5)}(t)+(d-4) t\left(t^{2}-1\right)^{2} \mathcal{C}_{l_{1}}^{(6)}(t), \\
& \hat{f}_{15}(t)=2(4-d)(2+d)(3+d) t \mathcal{C}_{l_{1}}^{(4)}(t)-4(d-4)(3+d) t^{2} \mathcal{C}_{l_{1}}^{(5)}(t)-2(d-4) t\left(t^{2}-1\right) \mathcal{C}_{l_{1}}^{(6)}(t), \\
& \hat{f}_{16}(t)=16(d-1)(1+d){ }_{2} t \mathcal{C}_{l_{1}}^{(3)}(t)+4(2+d)\left(-4 d+(-8+d(11+d)) t^{2}\right) \mathcal{C}_{l_{1}}^{(4)}(t) \\
& +8(-2+d(5+d)) t\left(t^{2}-1\right) \mathcal{C}_{l_{1}}^{(5)}(t)+4 d\left(t^{2}-1\right)^{2} \mathcal{C}_{l_{1}}^{(6)}(t)
\end{aligned}
$$

\section{Computation of the constants $\mathcal{S}_{\Delta \Delta_{12}}^{\lambda}$ from the shadow formalism}

In this section the constant

$$
\mathcal{S}_{\Delta \Delta_{12}}^{\lambda}=\frac{\left\langle\mathcal{O}_{1}\left(P_{1} ; \mathbf{Z}_{1}\right) \mathcal{O}_{2}\left(P_{2} ; \mathbf{Z}_{2}\right) \tilde{\mathcal{O}}\left(P_{3} ; \mathbf{Z}_{3}\right)\right\rangle}{\left.\left\langle\mathcal{O}_{1}\left(P_{1} ; \mathbf{Z}_{1}\right) \mathcal{O}_{2}\left(P_{2} ; \mathbf{Z}_{2}\right) \mathcal{O}\left(P_{3} ; \mathbf{Z}_{3}\right)\right\rangle\right|_{\Delta \rightarrow \tilde{\Delta}}}
$$


is computed. Previous known results are the cases \langle

In this appendix, the constant is computed for any three-point function that has a single tensor structure and obeys $\left|\lambda_{1}\right|+\left|\lambda_{2}\right|=|\lambda|-l_{1}$ (as discussed in section 3.1). We start in subsection D.1 by showing that $\mathcal{S}_{\Delta \Delta_{12}}^{\lambda}$ does not depend on the $\mathrm{SO}(d)$ irreps $\lambda_{1}$ and $\lambda_{2}$, by constructing a differential operator that transfers spin between the operators $\mathcal{O}_{1}$ and $\mathcal{O}_{2}$ without violating the condition $\left|\lambda_{1}\right|+\left|\lambda_{2}\right|=|\lambda|-l_{1}$. In the following subsections $\mathcal{S}_{\Delta \Delta_{12}}^{\lambda}$ is computed for the case of an arbitrary irrep $\lambda=\left(l_{1}, l_{2}, l_{3}, \ldots\right)$ and $\lambda_{1}=\bullet, \lambda_{2}=\left(l_{2}, l_{3}, \ldots\right)$.

\section{D.1 Spin transfer operator}

The shadow operator is

$$
\tilde{\mathcal{O}}\left(P_{3} ; \mathbf{Z}_{3}\right)=\left.\int D^{d} P_{0} \mathcal{O}\left(P_{0} ; \boldsymbol{\partial}_{\mathbf{Z}_{0}}\right) \pi_{\lambda}\left(\mathbf{Z}_{0} ; \boldsymbol{\partial}_{\overline{\mathbf{Z}}_{0}}\right)\left\langle\mathcal{O}\left(P_{0} ; \overline{\mathbf{Z}}_{0}\right) \mathcal{O}\left(P_{3} ; \mathbf{Z}_{3}\right)\right\rangle\right|_{\Delta \rightarrow \tilde{\Delta}}
$$

hence the three-point function of the shadow is

$$
\begin{aligned}
\left\langle\mathcal{O}_{1}\left(P_{1} ; \mathbf{Z}_{1}\right) \mathcal{O}_{2}\left(P_{2} ; \mathbf{Z}_{2}\right) \tilde{\mathcal{O}}\left(P_{3} ; \mathbf{Z}_{3}\right)\right\rangle=\int & D^{d} P_{0}\left\langle\mathcal{O}_{1}\left(P_{1} ; \mathbf{Z}_{1}\right) \mathcal{O}_{2}\left(P_{2} ; \mathbf{Z}_{2}\right) \mathcal{O}\left(P_{0} ; \boldsymbol{\partial}_{\mathbf{Z}_{0}}\right)\right\rangle \\
& \left.\pi_{\lambda}\left(\mathbf{Z}_{0} ; \boldsymbol{\partial}_{\overline{\mathbf{Z}}_{0}}\right)\left\langle\mathcal{O}\left(P_{0} ; \overline{\mathbf{Z}}_{0}\right) \mathcal{O}\left(P_{3} ; \mathbf{Z}_{3}\right)\right\rangle\right|_{\Delta \rightarrow \tilde{\Delta}}
\end{aligned}
$$

Our strategy will be to find a differential operator which transforms a three-point function with operators $\mathcal{O}, \mathcal{O}_{1}$ and $\mathcal{O}_{2}$ in the representations $\lambda=\left(l_{1}, l_{2}, \ldots, l_{h_{1}}\right)$ and $\lambda_{1}=\bullet$, $\lambda_{2}=\left(l_{2}, \ldots, l_{h_{1}}\right)$

$$
\left\langle\mathcal{O}_{1}\left(P_{1}\right) \mathcal{O}_{2}\left(P_{2}, \mathbf{Z}_{2}\right) \mathcal{O}\left(P_{3}, \mathbf{Z}_{3}\right)\right\rangle_{\text {enc }}=\frac{\left(V_{(3,12)}^{\left(Z_{31}\right)}\right)^{l_{1}}\left(H_{23}^{\left(Z_{21}, Z_{32}\right)}\right)^{l_{2}} \ldots\left(H_{23}^{\left(Z_{2\left(h_{1}-1\right)}, Z_{3 h_{1}}\right)}\right)^{l_{h_{1}}}}{\left(P_{13}\right)^{\frac{\Delta_{1}+\Delta-\Delta_{2}}{2}}\left(P_{23}\right)^{\frac{\Delta_{2}+\Delta-\Delta_{1}}{2}}\left(P_{12}\right)^{\frac{\Delta_{1}+\Delta_{2}-\Delta}{2}}}
$$

into another three-point function with a single tensor structure by transferring spin from the operator $\mathcal{O}_{2}$ to $\mathcal{O}_{1}$. This operator must decrease the homogeneity of a polynomial in $Z_{2 i}$ and increase it in $Z_{1 j}$ by one each, while not changing the homogeneity in $P_{1}$ and $P_{2}$. Furthermore, it must preserve the transverseness of the functions, that is

$$
F\left(P_{i}, Z_{i j}+c P_{i}\right)=F\left(P_{i}, Z_{i j}\right)
$$

This last requirement means that the operator should transfer terms of order

$$
\mathcal{O}\left(Z_{i j}^{2}, Z_{i j} \cdot P_{i}, P_{i}^{2}\right), \quad i \in\{1,2\}
$$

into terms of the same kind. Such an operator is given in terms of the differential operator derived in appendix $\mathrm{A}$ in $d+2$ dimensions (i.e. each $d$ in its definition must be replaced by $d+2)$,

$$
\mathcal{D}_{Z_{2 i}, Z_{1 j}, P_{2}, P_{1}}=\frac{\left(Z_{1 j} \cdot P_{2}\right) P_{1 A}-\left(P_{1} \cdot P_{2}\right) Z_{1 j A}}{P_{1} \cdot P_{2}} D_{Z_{2 i}, P_{2}}^{A}
$$


The term that is contracted into the operator $D_{Z_{2 i}, P_{2}}^{A}$ ensures transverseness in $Z_{1 j}$. By construction, the action on (D.4) must yield a transverse function

$$
\begin{aligned}
& \mathcal{D}_{Z_{2 i}, Z_{1 j}, P_{2}, P_{1}} \frac{\left(V_{(3,12)}^{\left(Z_{31}\right)}\right)^{l_{1}}\left(H_{23}^{\left(Z_{21}, Z_{32}\right)}\right)^{l_{2}} \ldots\left(H_{23}^{\left(Z_{2\left(h_{1}-1\right)}, Z_{3 h_{1}}\right)}\right)^{l_{h_{1}}}}{\left(P_{13}\right)^{\frac{1}{2}\left(\Delta_{1}+\Delta-\Delta_{2}\right)}\left(P_{23}\right)^{\frac{1}{2}\left(\Delta_{2}+\Delta-\Delta_{1}\right)}\left(P_{12}\right)^{\frac{1}{2}\left(\Delta_{1}+\Delta_{2}-\Delta\right)}} \propto \\
& \left(\frac{H_{13}^{\left(Z_{1 j}, Z_{3(i+1)}\right)}+c V_{(1,23)}^{\left(Z_{1 j}\right)} V_{(3,12)}^{\left(Z_{3(i+1)}\right)}}{H_{23}^{\left(Z_{2 i}, Z_{3(i+1)}\right)}}\right) \frac{\left(V_{(3,12)}^{\left(Z_{31}\right)}\right)^{l_{1}}\left(H_{23}^{\left(Z_{21}, Z_{32}\right)}\right)^{l_{2}} \ldots\left(H_{23}^{\left(Z_{2\left(h_{1}-1\right)}, Z_{3 h_{1}}\right)}\right)^{l_{h_{1}}}}{\left(P_{13}\right)^{\frac{1}{2}\left(\Delta_{1}+\Delta-\Delta_{2}\right)}\left(P_{23}\right)^{\frac{1}{2}\left(\Delta_{2}+\Delta-\Delta_{1}\right)}\left(P_{12}\right)^{\frac{1}{2}\left(\Delta_{1}+\Delta_{2}-\Delta\right)}} \\
& \quad+\mathcal{O}\left(P_{3}^{2}, P_{3} \cdot Z_{3 k}, Z_{3 k} \cdot Z_{3 l}\right),
\end{aligned}
$$

where $c$ is an unspecified constant. The term containing $V_{(3,12)}^{\left(Z_{3(i+1)}\right)}$ vanishes upon Young symmetrization with $\left(V_{(3,12)}^{\left(Z_{31}\right)}\right)^{l_{1}}$. By repeated use of such operators, any three-point function with a single tensor structure can be generated

$$
\begin{aligned}
\left\langle\mathcal{O}_{1}\left(P_{1}, \mathbf{Z}_{2}\right)\right. & \left.\mathcal{O}_{2}\left(P_{2}, \mathbf{Z}_{2}\right) \mathcal{O}\left(P_{3}, \mathbf{Z}_{3}\right)\right\rangle_{\text {full }} \propto \\
& \pi_{\lambda_{1}}\left(\mathbf{Z}_{1}, \boldsymbol{\partial}_{\overline{\mathbf{Z}}_{1}}\right) \pi_{\lambda_{2}}\left(\mathbf{Z}_{2}, \boldsymbol{\partial}_{\overline{\mathbf{Z}}_{2}}\right) \pi_{\lambda}\left(\mathbf{Z}_{3}, \boldsymbol{\partial}_{\overline{\mathbf{Z}}_{3}}\right) \\
& \mathcal{D}_{\bar{Z}_{2 i}, \bar{Z}_{1 j}, P_{2}, P_{1}} \ldots \mathcal{D}_{\bar{Z}_{2 k}, \bar{Z}_{1 l}, P_{2}, P_{1}}\left\langle\hat{\mathcal{O}}_{1}\left(P_{1}\right) \hat{\mathcal{O}}_{2}\left(P_{2}, \overline{\mathbf{Z}}_{2}\right) \mathcal{O}\left(P_{3}, \overline{\mathbf{Z}}_{3}\right)\right\rangle_{\text {enc }}
\end{aligned}
$$

where $\hat{\mathcal{O}}_{1}\left(P_{1}\right)$ is in the scalar representation $\hat{\lambda}_{1}=\bullet$ and $\hat{\mathcal{O}}_{2}\left(P_{2}, \overline{\mathbf{Z}}_{2}\right)$ in an irrep $\hat{\lambda}_{2}$ satisfying $\left|\hat{\lambda}_{2}\right|=\left|\lambda_{1}\right|+\left|\lambda_{2}\right|$. In order to compute $\mathcal{S}_{\Delta \Delta_{12}}^{\lambda}$ one can insert (D.9) into (D.1). The action of the operator on both three-point functions is obviously the same, and the resulting $\mathcal{S}_{\Delta \Delta_{12}}^{\lambda}$ is the same that one gets when using the three-point function given in (D.4). In the next two subsections, $\mathcal{S}_{\Delta \Delta_{12}}^{\lambda}$ is computed using this three-point function.

\section{D.2 Young diagrams with two rows}

The computations here are done using the conventions of [42] to allow reusing some of their computations. We start with the case of $\lambda=\left(l_{1}, l_{2}\right)$. To compute the constant we will consider a three-point function of an operator $\mathcal{O}$ in this representation with a scalar $\mathcal{O}_{1}$ and a symmetric tensor $\mathcal{O}_{2}$ of spin $l_{2}$. This correlator can be written as

$$
\left\langle\mathcal{O}_{1}\left(x_{1}\right) \mathcal{O}_{2}\left(x_{2}, z_{2}\right) \mathcal{O}\left(x_{3}, \mathbf{z}_{3}\right)\right\rangle=\frac{\pi_{\lambda}\left(\mathbf{z}_{3} ; \boldsymbol{\partial}_{\overline{\mathbf{z}}_{3}}\right)\left(k^{(312)}\left(\bar{z}_{31}\right)\right)^{l_{1}}\left(m^{(23)}\left(z_{2}, \bar{z}_{32}\right)\right)^{l_{2}}}{\left(x_{13}^{2}\right)^{\frac{\Delta_{1}-\Delta_{2}+\Delta_{3}}{2}}\left(x_{23}^{2}\right)^{\frac{-\Delta_{1}+\Delta_{2}+\Delta_{3}}{2}}\left(x_{12}^{2}\right)^{\frac{\Delta_{1}+\Delta_{2}-\Delta_{3}}{2}}},
$$

where the building blocks $m^{(i j)}$ and $k^{(i j k)}$ are physical space variants of the building blocks $H_{i j}$ and $V_{i, j k}$ defined in (3.12), given by

$$
m_{a b}^{(i j)}=\delta_{a b}-\frac{2}{x_{i j}^{2}}\left(x_{i j}\right)_{a}\left(x_{i j}\right)_{b}, \quad k_{a}^{(i j k)}=\frac{x_{i j}^{2}\left(x_{i k}\right)_{a}-x_{i k}^{2}\left(x_{i j}\right)_{a}}{\left(x_{i j}^{2} x_{i k}^{2} x_{j k}^{2}\right)^{1 / 2}} .
$$

We will also use the notation

$$
m^{(i j)}\left(z_{k}, z_{l}\right)=z_{k}^{a} m_{a b}^{(i j)} z_{l}^{b}, \quad k^{(i j k)}(z)=k_{a}^{(i j k)} z^{a} .
$$

To compute the ratio in (D.1) it is not necessary to keep track of terms containing $z_{31}^{2}, z_{32}^{2}$ or $z_{31} \cdot z_{32}$. Those will be collectively denoted by $\mathcal{O}\left(z_{3 i} \cdot z_{3 j}\right)$. Furthermore, it is enough to consider the term of order $l_{2}$ in $z_{2} \cdot z_{32}$, so terms of order $\mathcal{O}\left(\left(z_{2} \cdot z_{32}\right)^{l_{2}-1}\right)$ can be dropped. 
We need to compute the three-point function of the shadow operator. This is given by

$$
\begin{aligned}
\left\langle\mathcal{O}_{1}\left(x_{1}\right) \mathcal{O}_{2}\left(x_{2}, z_{2}\right) \tilde{\mathcal{O}}\left(x_{3}, \mathbf{z}_{3}\right)\right\rangle & \\
= & \frac{1}{l_{1} ! l_{2} !} \pi_{\lambda}\left(\mathbf{z}_{3} ; \boldsymbol{\partial}_{\overline{\mathbf{z}}_{3}}\right) \int \frac{d^{d} x_{0}}{\left(x_{03}^{2}\right)^{d-\Delta}\left(x_{01}^{2}\right)^{\frac{\Delta_{1}-\Delta_{2}+\Delta}{2}}\left(x_{02}^{2}\right)^{\frac{-\Delta_{1}+\Delta_{2}+\Delta}{2}}\left(x_{12}^{2}\right)^{\frac{\Delta_{1}+\Delta_{2}-\Delta}{2}}} \\
& \left(m^{(30)}\left(\bar{z}_{31}, \partial_{z_{01}}\right)\right)^{l_{1}}\left(m^{(30)}\left(\bar{z}_{32}, \partial_{z_{02}}\right)\right)^{l_{2}} \pi_{\lambda}\left(\mathbf{z}_{0} ; \boldsymbol{\partial}_{\overline{\mathbf{z}}_{0}}\right)\left(k^{(012)}\left(\bar{z}_{01}\right)\right)^{l_{1}}\left(m^{(02)}\left(\bar{z}_{02}, z_{2}\right)\right)^{l_{2}} \\
= & \pi_{\lambda}\left(\mathbf{z}_{3} ; \boldsymbol{\partial}_{\overline{\mathbf{z}}_{3}}\right) \int \frac{d^{d} x_{0}}{\left(x_{03}^{2}\right)^{d-\Delta}} \frac{\left(y\left(\bar{z}_{31}\right)\right)^{l_{1}}\left(y_{2}\left(\bar{z}_{32}, z_{2}\right)\right)^{l_{2}}}{\left(x_{01}^{2}\right)^{\frac{\Delta_{1}-\Delta_{2}+\Delta}{2}}\left(x_{02}^{2}\right)^{\frac{-\Delta_{1}+\Delta_{2}+\Delta}{2}}\left(x_{12}^{2}\right)^{\frac{\Delta_{1}+\Delta_{2}-\Delta}{2}}}
\end{aligned}
$$

where we defined

$$
\begin{aligned}
y\left(z_{31}\right) & =z_{31}^{a} m_{a b}^{(30)} k^{(012) b} \\
& =\left(\sqrt{\frac{x_{01}^{2} x_{23}^{2}}{x_{03}^{2} x_{12}^{2}}}-\frac{x_{02}^{2} x_{13}^{2}}{\sqrt{x_{01}^{2} x_{03}^{2} x_{12}^{2} x_{23}^{2}}}\right) k^{(302)}\left(z_{31}\right)+\sqrt{\frac{x_{02}^{2} x_{13}^{2}}{x_{01}^{2} x_{23}^{2}}} k^{(312)}\left(z_{31}\right), \\
y_{2}\left(z_{32}, z_{2}\right) & =z_{32}^{a} m_{a b}^{(30)} m_{b c}^{(02)} z_{2}^{c} \\
& =m^{(32)}\left(z_{32}, z_{2}\right)-2 k^{(302)}\left(z_{32}\right) k^{(203)}\left(z_{2}\right) .
\end{aligned}
$$

The reason why the trace subtracting terms of the projector $\pi_{\lambda}\left(\mathbf{z}_{0} ; \boldsymbol{\partial}_{\overline{\mathbf{z}}_{0}}\right)$ in (D.13) do not contribute is that $m_{a c}^{(30)} m_{b c}^{(30)}=\delta_{a b}$, so these terms are annihilated by the other projector. Not keeping track of terms containing $z_{31}^{2}, z_{32}^{2}$ or $z_{31} \cdot z_{32}$ means that the trace subtracting terms of the other projector can be ignored as well and we can use instead the Young symmetrizer

$$
Y_{\lambda}\left(z_{1}, z_{2} ; \bar{z}_{1}, \bar{z}_{2}\right)=\left.\pi_{\lambda}\left(z_{1}, z_{2} ; \bar{z}_{1}, \bar{z}_{2}\right)\right|_{z_{i}^{2}=z_{1} \cdot z_{2}=\bar{z}_{i}^{2}=\bar{z}_{1} \cdot \bar{z}_{2}=0}
$$

leading to

$$
\begin{aligned}
& \left\langle\mathcal{O}_{1}\left(x_{1}\right) \mathcal{O}_{2}\left(x_{2}, z_{2}\right) \tilde{\mathcal{O}}\left(x_{3}, \mathbf{z}_{3}\right)\right\rangle= \\
& Y_{\lambda}\left(\mathbf{z}_{3} ; \boldsymbol{\partial}_{\overline{\mathbf{z}}_{3}}\right) \int \frac{d^{d} x_{0}}{\left(x_{03}^{2}\right)^{d-\Delta}} \frac{\left(y\left(\bar{z}_{31}\right)\right)^{l_{1}}\left(y_{2}\left(\bar{z}_{32}, z_{2}\right)\right)^{l_{2}}}{\left(x_{01}^{2}\right)^{\frac{\Delta_{1}-\Delta_{2}+\Delta}{2}}\left(x_{02}^{2}\right)^{\frac{-\Delta_{1}+\Delta_{2}+\Delta}{2}}\left(x_{12}^{2}\right)^{\frac{\Delta_{1}+\Delta_{2}-\Delta}{2}}}+\mathcal{O}\left(z_{3 i} \cdot z_{3 j}\right) .
\end{aligned}
$$

One needs to compute the conformal integral

$$
I_{\alpha, \beta, \gamma}^{n, m}\left(x_{1}, x_{2}, x_{3}, z_{31}, z_{32}, z_{2}\right)=\int \frac{d^{d} x_{0}\left(k^{(302)}\left(z_{31}\right)\right)^{n}\left(k^{(302)}\left(z_{32}\right) k^{(203)}\left(z_{2}\right)\right)^{m}}{\left(x_{01}^{2}\right)^{\alpha}\left(x_{02}^{2}\right)^{\beta}\left(x_{03}^{2}\right)^{\gamma}}
$$

which is done in subsection D.4 below. Next we do a trinomial expansion of $y\left(z_{31}\right)$ and a binomial expansion of $y_{2}\left(z_{32}, z_{2}\right)$ in (D.16). Note that we consider only the term proportional to $\left(z_{2} \cdot z_{32}\right)^{l_{2}}$, for which the action of the Young symmetrizer results only in the 
factor $S_{l_{1}, k+b, i}$, which will be explained below

$$
\begin{aligned}
\left\langle\mathcal{O}_{1}\left(x_{1}\right)\right. & \left.\mathcal{O}_{2}\left(x_{2}, z_{2}\right) \tilde{\mathcal{O}}\left(x_{3}, \mathbf{z}_{3}\right)\right\rangle \\
= & \sum_{k=0}^{l_{1}} \sum_{b=0}^{l_{1}-k} \frac{l_{1} !}{k ! b !\left(l_{1}-k-b\right) !}(-1)^{b} \sum_{i=0}^{l_{2}} \frac{l_{2} !}{i !\left(l_{2}-i\right) !}(-2)^{i} S_{l_{1}, k+b, i} \\
& \left(x_{12}^{2}\right)^{\frac{-\Delta_{1}-\Delta_{2}+\Delta-k-b}{2}}\left(x_{13}^{2}\right)^{\frac{l_{1}-k+b}{2}}\left(x_{23}^{2}\right)^{k-\frac{l_{1}}{2}}\left(k^{(312)}\left(z_{31}\right)\right)^{l_{1}-k-b}\left(z_{2} \cdot z_{32}\right)^{l_{2}-i} \\
& I_{\frac{1}{2}\left(\Delta_{12}+\Delta+l_{1}-2 k\right), \frac{1}{2}\left(-\Delta_{12}+\Delta-l_{1}+k-b\right), d-\Delta+\frac{k+b}{2}}^{k+i}\left(x_{1}, x_{2}, x_{3}, z_{31}, z_{32}, z_{2}\right) \\
& +\mathcal{O}\left(z_{3 i} \cdot z_{3 j}\right)+\mathcal{O}\left(\left(z_{2} \cdot z_{32}\right)^{l_{2}-1}\right) \\
= & \pi^{d / 2}\left(k^{(312)}\left(z_{31}\right)\right)^{l_{1}}\left(z_{2} \cdot z_{32}\right)^{l_{2}}\left(x_{12}^{2}\right)^{\frac{\tilde{\Delta}-\Delta_{1}-\Delta_{2}}{2}}\left(x_{13}^{2}\right)^{\frac{\Delta_{2}-\Delta_{1}-\tilde{\Delta}}{2}}\left(x_{23}^{2}\right)^{\frac{\Delta_{1}-\Delta_{2}-\tilde{\Delta}}{2}} \\
& \sum_{i=0}^{l_{2}} \sum_{k=0}^{l_{1}-i l_{1}-k-i} \sum_{b=0} \frac{\left(l_{1}-i\right) ! l_{2} !}{k ! b !\left(l_{1}-k-b-i\right) !\left(l_{2}-i\right) !}(-1)^{b+i} \\
& \frac{\Gamma\left(\frac{1}{2}\left(d-\Delta, \Delta-\Delta-l_{1}\right)+k+i\right) \Gamma\left(\frac{1}{2}\left(d+\Delta_{12}-\Delta+l_{1}\right)+b\right) \Gamma\left(\Delta-\frac{d}{2}\right)}{\Gamma\left(\frac{1}{2}\left(\Delta_{12}+\Delta+l_{1}\right)-k\right) \Gamma\left(\frac{1}{2}\left(-\Delta_{12}+\Delta-l_{1}\right)+k+i\right) \Gamma(d-\Delta+k+b+i)} \\
& +\mathcal{O}\left(z_{3 i} \cdot z_{3 j}\right)+\mathcal{O}\left(\left(z_{2} \cdot z_{32}\right)^{l_{2}-1}\right) \\
= & \pi^{d / 2}\left(k^{(312)}\left(z_{31}\right)\right)^{l_{1}}\left(z_{2} \cdot z_{32}\right)^{l_{2}}\left(x_{12}^{2}\right)^{\frac{\tilde{\Delta}-\Delta_{1}-\Delta_{2}}{2}}\left(x_{13}^{2}\right)^{\frac{\Delta_{2}-\Delta_{1}-\tilde{\Delta}}{2}}\left(x_{23}^{2} \frac{\Delta_{1}-\Delta_{2}-\tilde{\Delta}}{2}\right. \\
& \frac{(\Delta-2)_{l_{1}+1}}{\Gamma\left(\Delta-\frac{d}{2}\right) \Gamma\left(\frac{1}{2}\left(d+\Delta \Delta_{12}-\Delta+l_{1}\right)\right) \Gamma\left(\frac{1}{2}\left(d-\Delta_{12}-\Delta+l_{1}\right)\right)} \\
& \left(\Delta-2+l_{2}\right) \\
& +\mathcal{O}\left(z_{3 i} \cdot z_{3 j}\right)+\mathcal{O}\left(\left(z_{2} \cdot z_{32}\right)^{l_{2}-1}\right) .
\end{aligned}
$$

The sums were evaluated by using first that

$$
\sum_{b=0}^{N} \frac{N !}{b !(N-b) !}(-1)^{b} \frac{\Gamma(x+b)}{\Gamma(y+b)}=\frac{\Gamma(x) \Gamma(y-x+N)}{\Gamma(y+N) \Gamma(y-x)},
$$

then

$$
\sum_{k=0}^{N} \frac{N !}{k !(N-k) !} \frac{1}{\Gamma(x+k) \Gamma(y-k)}=\frac{\Gamma(x+y+N-1)}{\Gamma(x+N) \Gamma(y) \Gamma(x+y-1)},
$$

and finally

$$
\sum_{i=1}^{l_{2}} \frac{l_{2} !(-1)^{i}}{\left(l_{2}-i\right) !} \frac{\Gamma\left(\Delta-1+l_{1}\right)}{\Gamma(\Delta-1+i)}=\frac{(\Delta-2) l_{1}+1}{\left(\Delta-2+l_{2}\right)} .
$$

The factor $S_{l_{1}, k+b, i}$ appearing in (D.18) is given by

$$
S_{l_{1}, k+b, i}=\frac{\left(l_{1}-k-b\right)_{(i)}}{\left(l_{1}\right)_{(i)}},
$$

where we used the following notation for the falling factorial

$$
(x)_{(n)} \equiv(x-n+1)_{n}=(x)(x-1) \ldots(x-n+1) .
$$


This factor arises from the Young symmetrization of the tensor encoded by $\left(z_{31}, z_{32}\right)$. This can be understood by drawing birdtracks, where the $\left(z_{31}, z_{32}\right)$ are antisymmetrized according to the Young symmetrizer. The following birdtracks represent the term

$$
\left(k^{(312)}\left(z_{31}\right)\right)^{l_{1}-k-b}\left(k^{(302)}\left(z_{31}\right)\right)^{k+b}\left(k^{(302)}\left(z_{32}\right)\right)^{i},
$$

which appears in (D.18) before integration. The contraction with multiple copies of $k_{a}^{(302)}$ results in a symmetrization which cancels all terms in which two antisymmetrized indices get symmetrized. Consider for example the case with $i=1$, where the cancellations lead to a factor of $S_{l_{1}, k+b, 1}=\frac{l_{1}-k-b}{l_{1}}$,
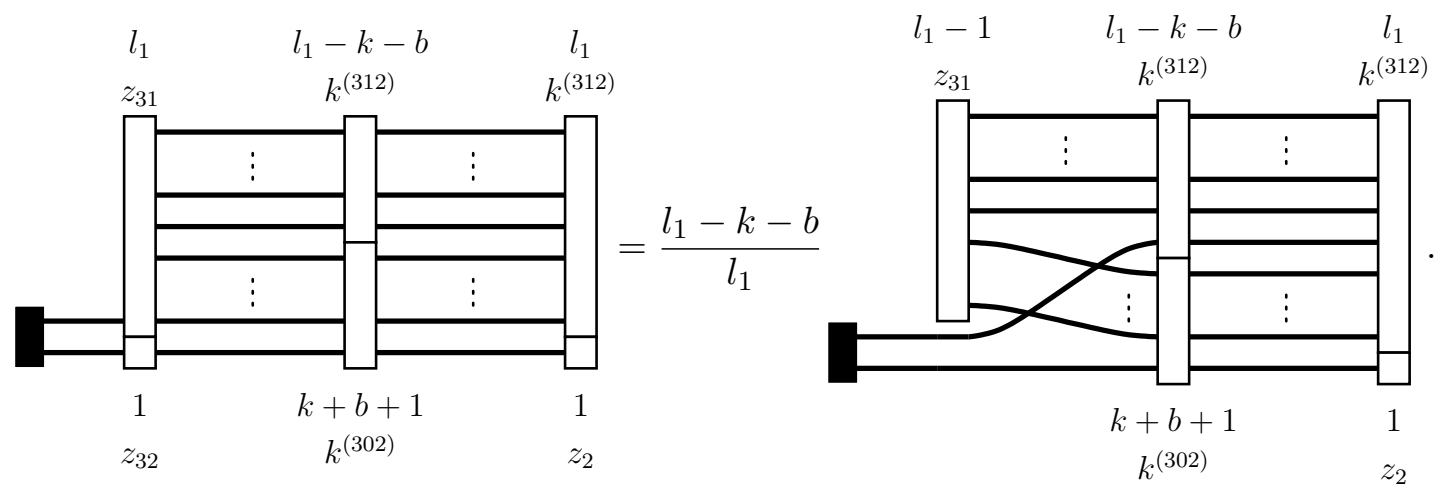

Consider also the next case $i=2$,
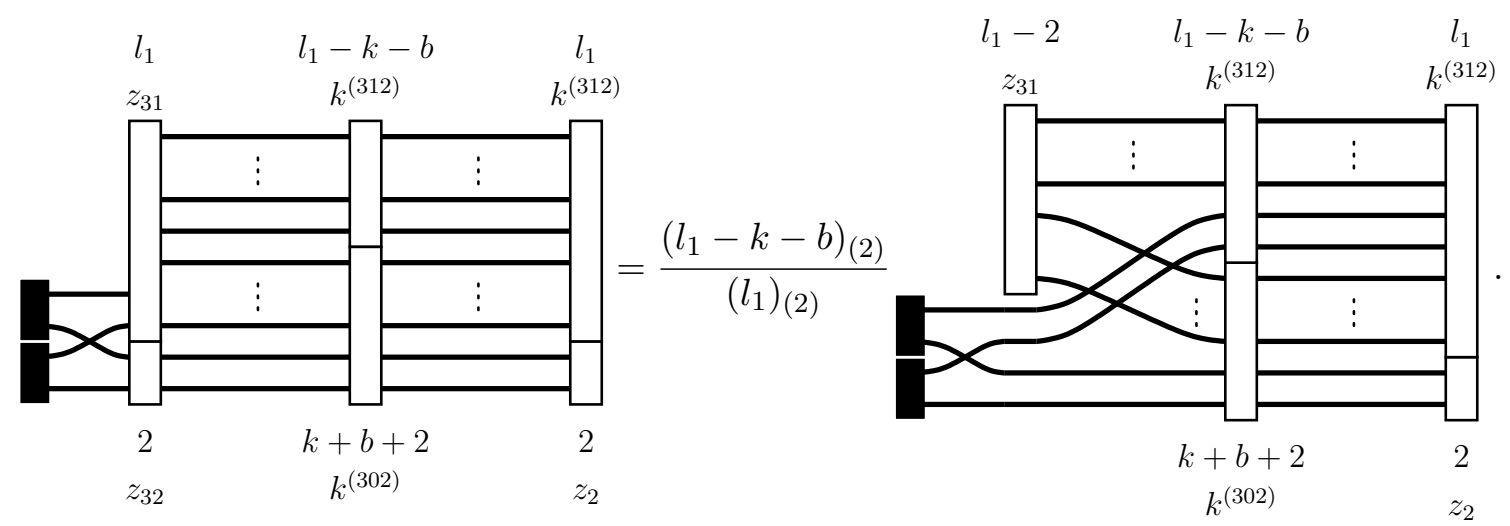

The constant $\mathcal{S}$ is now found by comparing (D.18) with the corresponding term in (D.10)

$$
\mathcal{S}_{\Delta \Delta_{12}}^{\left(l_{1}, l_{2}\right)}=\pi^{d / 2} \frac{(\Delta-2)_{l_{1}+1}}{\left(\Delta-2+l_{2}\right)} \frac{\Gamma\left(\Delta-\frac{d}{2}\right) \Gamma\left(\frac{1}{2}\left(\tilde{\Delta}+\Delta_{12}+l_{1}\right)\right) \Gamma\left(\frac{1}{2}\left(\tilde{\Delta}-\Delta_{12}+l_{1}\right)\right)}{\Gamma\left(\tilde{\Delta}+l_{1}\right) \Gamma\left(\frac{1}{2}\left(\Delta+\Delta_{12}+l_{1}\right)\right) \Gamma\left(\frac{1}{2}\left(\Delta-\Delta_{12}+l_{1}\right)\right)} .
$$




\section{D.3 Arbitrary representations}

As a next example we will compute the constant for the representations $\lambda=\left(l_{1}, l_{2}, l_{3}\right)$, $\lambda_{1}=\bullet, \lambda_{2}=\left(l_{2}, l_{3}\right)$. From the derivation it will also be clear what the result is for arbitrary $\lambda$. The three-point function is in this case

$$
\begin{aligned}
\left\langle\mathcal{O}_{1}\left(x_{1}\right) \mathcal{O}_{2}\left(x_{2}, \mathbf{z}_{2}\right) \mathcal{O}\left(x_{3}, \mathbf{z}_{3}\right)\right\rangle_{\text {full }} & \\
= & \pi_{\lambda}\left(\mathbf{z}_{2} ; \boldsymbol{\partial}_{\overline{\mathbf{z}}_{2}}\right) \pi_{\lambda}\left(\mathbf{z}_{3} ; \boldsymbol{\partial}_{\overline{\mathbf{z}}_{3}}\right) \frac{\left(k^{(312)}\left(\bar{z}_{31}\right)\right)^{l_{1}}\left(m^{(23)}\left(\bar{z}_{21}, \bar{z}_{32}\right)\right)^{l_{2}}\left(m^{(23)}\left(\bar{z}_{22}, \bar{z}_{33}\right)\right)^{l_{3}}}{\left(x_{13}^{2}\right)^{\frac{\Delta_{1}-\Delta_{2}+\Delta_{3}}{2}}\left(x_{23}^{2}\right)^{\frac{-\Delta_{1}+\Delta_{2}+\Delta_{3}}{2}}\left(x_{12}^{2}\right)^{\frac{\Delta_{1}+\Delta_{2}-\Delta_{3}}{2}}} \\
= & \pi_{\lambda}\left(\mathbf{z}_{3} ; \boldsymbol{\partial}_{\overline{\mathbf{z}}_{3}}\right) \frac{\left(k^{(312)}\left(\bar{z}_{31}\right)\right)^{l_{1}}\left(m^{(23)}\left(z_{21}, \bar{z}_{32}\right)\right)^{l_{2}}\left(m^{(23)}\left(z_{22}, \bar{z}_{33}\right)\right)^{l_{3}}}{\left(x_{13}^{2}\right)^{\frac{\Delta_{1}-\Delta_{2}+\Delta_{3}}{2}}\left(x_{23}^{2}\right)^{\frac{-\Delta_{1}+\Delta_{2}+\Delta_{3}}{2}}\left(x_{12}^{2}\right)^{\frac{\Delta_{1}+\Delta_{2}-\Delta_{3}}{2}}} \\
& +\mathcal{O}\left(z_{2 i} \cdot z_{2 j}\right)+\mathcal{O}\left(\left(z_{21} \cdot z_{32}\right)^{l_{2}-1}\right)+\mathcal{O}\left(\left(z_{22} \cdot z_{33}\right)^{l_{3}-1}\right) .
\end{aligned}
$$

The three-point function of the shadow operator is then

$$
\begin{aligned}
& \left\langle\mathcal{O}_{1}\left(x_{1}\right) \mathcal{O}_{2}\left(x_{2}, \mathbf{z}_{2}\right) \tilde{\mathcal{O}}\left(x_{3}, \mathbf{z}_{3}\right)\right\rangle_{\text {full }}= \\
& \quad \int \frac{d^{d} x_{0}}{\left(x_{03}^{2}\right)^{d-\Delta}} \frac{Y_{\lambda}\left(\mathbf{z}_{3} ; \boldsymbol{\partial}_{\overline{\mathbf{z}}_{31}}\right)\left(y\left(\bar{z}_{31}\right)\right)^{l_{1}}\left(y_{2}\left(\bar{z}_{32}, z_{21}\right)\right)^{l_{2}}\left(y_{2}\left(\bar{z}_{33}, z_{22}\right)\right)^{l_{3}}}{\left(x_{01}^{2}\right)^{\frac{\Delta_{1}-\Delta_{2}+\Delta}{2}}\left(x_{02}^{2}\right)^{\frac{-\Delta_{1}+\Delta_{2}+\Delta}{2}}\left(x_{12}^{2}\right)^{\frac{\Delta_{1}+\Delta_{2}-\Delta}{2}}} \\
& \quad+\mathcal{O}\left(z_{2 i} \cdot z_{2 j}\right)+\mathcal{O}\left(z_{3 i} \cdot z_{3 j}\right)+\mathcal{O}\left(\left(z_{21} \cdot z_{32}\right)^{l_{2}-1}\right)+\mathcal{O}\left(\left(z_{22} \cdot z_{33}\right)^{l_{3}-1}\right) .
\end{aligned}
$$

To compute this expression one needs the following integral, which can be immediately read off from the result (D.36)

$$
\begin{aligned}
& I_{\alpha, \beta, \gamma}^{n, m, o}\left(x_{1}, x_{2}, x_{3}, z_{31}, z_{32}, z_{21}, z_{33}, z_{22}\right) \\
& \equiv \int \frac{d^{d} x_{0}\left(k^{(302)}\left(z_{31}\right)\right)^{n}\left(k^{(302)}\left(z_{32}\right) k^{(203)}\left(z_{21}\right)\right)^{m}\left(k^{(302)}\left(z_{33}\right) k^{(203)}\left(z_{22}\right)\right)^{o}}{\left(x_{01}^{2}\right)^{\alpha}\left(x_{02}^{2}\right)^{\beta}\left(x_{03}^{2}\right)^{\gamma}} \\
&= \pi^{d / 2} \frac{m ! o !}{2^{m+o}} \frac{\Gamma\left(\frac{d}{2}-\alpha+m+o\right) \Gamma\left(\frac{d}{2}-\beta+\frac{n}{2}\right) \Gamma\left(\frac{d}{2}-\gamma+\frac{n}{2}\right)}{\Gamma(\alpha) \Gamma\left(\beta+\frac{n}{2}+m+o\right) \Gamma\left(\gamma+\frac{n}{2}+m+o\right)} \\
&\left(x_{23}^{2}\right)^{\alpha-\frac{d}{2}}\left(x_{13}^{2}\right)^{\beta-\frac{d}{2}}\left(x_{12}^{2}\right)^{\gamma-\frac{d}{2}}\left(k^{(312)}\left(z_{31}\right)\right)^{n}\left(z_{21} \cdot z_{32}\right)^{m}\left(z_{22} \cdot z_{33}\right)^{o} \\
&+\mathcal{O}\left(z_{3 i} \cdot z_{3 j}\right)+\mathcal{O}\left(\left(z_{21} \cdot z_{32}\right)^{m-1}\right)+\mathcal{O}\left(\left(z_{22} \cdot z_{33}\right)^{o-1}\right) .
\end{aligned}
$$


Next, the computation of (D.18) is repeated

$$
\begin{aligned}
& \left\langle\mathcal{O}_{1}\left(x_{1}\right) \mathcal{O}_{2}\left(x_{2}, \mathbf{z}_{2}\right) \tilde{\mathcal{O}}\left(x_{3}, \mathbf{z}_{3}\right)\right\rangle_{\text {full }} \\
& =\sum_{k=0}^{l_{1}} \sum_{b=0}^{l_{1}-k} \frac{l_{1} !}{k ! b !\left(l_{1}-k-b\right) !}(-1)^{b} \sum_{i=0}^{l_{2}} \frac{l_{2} !}{i !\left(l_{2}-i\right) !}(-2)^{i} \sum_{j=0}^{l_{3}} \frac{l_{3} !}{j !\left(l_{3}-j\right) !}(-2)^{j} S_{l_{1}, k+b, i+j} S_{l_{2}, i, j} \\
& \left(x_{12}^{2}\right)^{\frac{-\Delta_{1}-\Delta_{2}+\Delta-k-b}{2}}\left(x_{13}^{2}\right)^{\frac{l_{1}-k+b}{2}}\left(x_{23}^{2}\right)^{k-\frac{l_{1}}{2}}\left(k^{(312)}\left(z_{31}\right)\right)^{l_{1}-k-b}\left(z_{21} \cdot z_{32}\right)^{l_{2}-i}\left(z_{22} \cdot z_{33}\right)^{l_{3}-j} \\
& I_{\frac{1}{2}\left(\Delta_{12}+\Delta+l_{1}-2 k\right), \frac{1}{2}\left(-\Delta_{12}+\Delta-l_{1}+k-b\right), d-\Delta+\frac{k+b}{2}}^{k+,}\left(x_{1}, x_{2}, x_{3}, z_{31}, z_{32}, z_{21}, z_{33}, z_{22}\right) \\
& +\mathcal{O}\left(z_{2 i} \cdot z_{2 j}\right)+\mathcal{O}\left(z_{3 i} \cdot z_{3 j}\right)+\mathcal{O}\left(\left(z_{21} \cdot z_{32}\right)^{l_{2}-1}\right)+\mathcal{O}\left(\left(z_{22} \cdot z_{33}\right)^{l_{3}-1}\right) \\
& =\left(k^{(312)}\left(z_{31}\right)\right)^{l_{1}}\left(z_{21} \cdot z_{32}\right)^{l_{2}}\left(z_{22} \cdot z_{33}\right)^{l_{3}}\left(x_{12}^{2}\right)^{\frac{\tilde{\Delta}-\Delta_{1}-\Delta_{2}}{2}}\left(x_{13}^{2}\right)^{\frac{\Delta_{2}-\Delta_{1}-\tilde{\Delta}}{2}}\left(x_{23}^{2}\right)^{\frac{\Delta_{1}-\Delta_{2}-\tilde{\Delta}}{2}} \\
& \pi^{d / 2} \sum_{j=0}^{l_{3}} \sum_{i=0}^{l_{2}-j} \sum_{k=0}^{l_{1}-i-j} \sum_{b=0}^{l_{1}-k-i-j} \frac{\left(l_{1}-i-j\right) !\left(l_{2}-j\right) ! l_{3} !}{k ! b !\left(l_{1}-k-b-i-j\right) !\left(l_{2}-i-j\right) !\left(l_{3}-j\right) !}(-1)^{b+i+j} \\
& \frac{\Gamma\left(\frac{1}{2}\left(d-\Delta_{12}-\Delta-l_{1}\right)+k+i+j\right) \Gamma\left(\frac{1}{2}\left(d+\Delta_{12}-\Delta+l_{1}\right)+b\right) \Gamma\left(\Delta-\frac{d}{2}\right)}{\Gamma\left(\frac{1}{2}\left(\Delta_{12}+\Delta+l_{1}\right)-k\right) \Gamma\left(\frac{1}{2}\left(-\Delta_{12}+\Delta-l_{1}\right)+k+i+j\right) \Gamma(d-\Delta+k+b+i+j)} \\
& +\mathcal{O}\left(z_{2 i} \cdot z_{2 j}\right)+\mathcal{O}\left(z_{3 i} \cdot z_{3 j}\right)+\mathcal{O}\left(\left(z_{21} \cdot z_{32}\right)^{l_{2}-1}\right)+\mathcal{O}\left(\left(z_{22} \cdot z_{33}\right)^{l_{3}-1}\right) \\
& =\left(k^{(312)}\left(z_{31}\right)\right)^{l_{1}}\left(z_{21} \cdot z_{32}\right)^{l_{2}}\left(z_{22} \cdot z_{33}\right)^{l_{3}}\left(x_{12}^{2}\right)^{\frac{\tilde{\Delta}-\Delta_{1}-\Delta_{2}}{2}}\left(x_{13}^{2}\right)^{\frac{\Delta_{2}-\Delta_{1}-\tilde{\Delta}}{2}}\left(x_{23}^{2}\right)^{\frac{\Delta_{1}-\Delta_{2}-\tilde{\Delta}}{2}} \\
& \pi^{d / 2} \frac{(\Delta-3)_{l_{1}+2}}{\left(\Delta-2+l_{2}\right)\left(\Delta-3+l_{3}\right)} \frac{\Gamma\left(\Delta-\frac{d}{2}\right) \Gamma\left(\frac{1}{2}\left(d+\Delta_{12}-\Delta+l_{1}\right)\right) \Gamma\left(\frac{1}{2}\left(d-\Delta_{12}-\Delta+l_{1}\right)\right)}{\Gamma\left(d-\Delta+l_{1}\right) \Gamma\left(\frac{1}{2}\left(\Delta_{12}+\Delta+l_{1}\right)\right) \Gamma\left(\frac{1}{2}\left(-\Delta_{12}+\Delta+l_{1}\right)\right)} \\
& +\mathcal{O}\left(z_{2 i} \cdot z_{2 j}\right)+\mathcal{O}\left(z_{3 i} \cdot z_{3 j}\right)+\mathcal{O}\left(\left(z_{21} \cdot z_{32}\right)^{l_{2}-1}\right)+\mathcal{O}\left(\left(z_{22} \cdot z_{33}\right)^{l_{3}-1}\right) \text {. }
\end{aligned}
$$

The only non-trivial new ingredient here is the factor $S_{l_{2}, i, j}$. Since the vectors $z_{31}$ are treated as before (with the factor $S_{l_{1}, k+b, i+j}$ ), consider only the vectors $z_{32}$ and $z_{33}$. They appear in the form of

$$
\left(z_{21} \cdot z_{32}\right)^{l_{2}-i}\left(z_{22} \cdot z_{33}\right)^{l_{3}-j}\left(k^{(302)}\left(z_{32}\right)\right)^{i}\left(k^{(302)}\left(z_{33}\right)\right)^{j} .
$$

One can now draw a birdtrack containing only the antisymmetrizations of the $j$ copies of $z_{33}$ that are contracted to $k^{(302)}$. All of the indices that are antisymmetrized with those cannot be contracted to $k^{(302)}$ as well. Consider for example the case $j=2$,

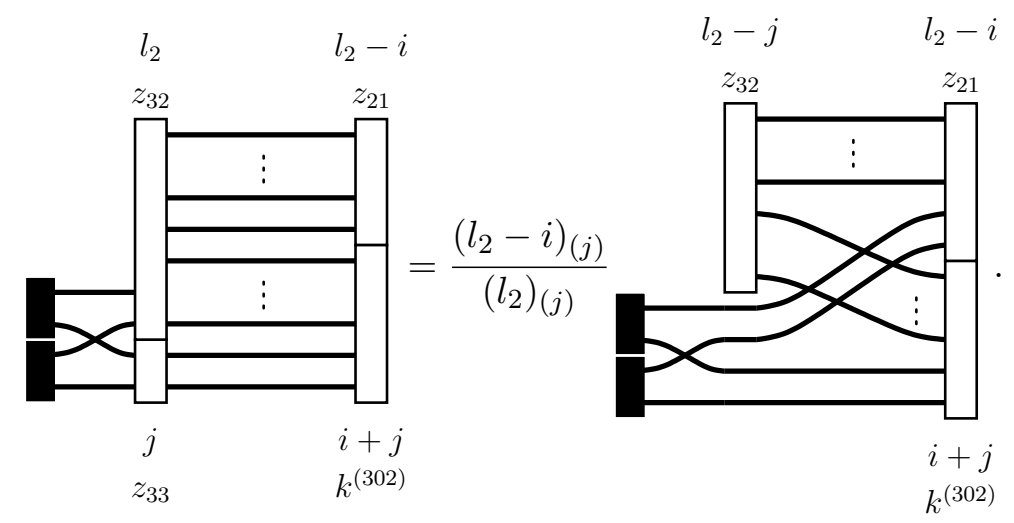

Hence the factor $S_{l_{2}, i, j}=\frac{\left(l_{2}-i\right)_{(j)}}{\left(l_{2}\right)_{(j)}}$ has to be included. 
By now it is clear that the computation for Young diagrams with more rows will work out analogously, and that the constant relating a three-point functions of an operator and the one of its shadow for a Young diagram with row lengths $\left(l_{1}, l_{2}, \ldots\right)$ and column heights $\left(h_{1}, h_{2}, \ldots\right)$ is

$$
\begin{aligned}
\mathcal{S}_{\Delta \Delta_{12}}^{\lambda}= & \pi^{d / 2} \frac{\left(\Delta-h_{1}\right)_{l_{1}+h_{1}-1}}{\prod_{i=2}^{h_{1}}\left(\Delta-i+l_{i}\right)} \frac{\Gamma\left(\Delta-\frac{d}{2}\right) \Gamma\left(\frac{1}{2}\left(\tilde{\Delta}+\Delta_{12}+l_{1}\right)\right) \Gamma\left(\frac{1}{2}\left(\tilde{\Delta}-\Delta_{12}+l_{1}\right)\right)}{\Gamma\left(\tilde{\Delta}+l_{1}\right) \Gamma\left(\frac{1}{2}\left(\Delta+\Delta_{12}+l_{1}\right)\right) \Gamma\left(\frac{1}{2}\left(\Delta-\Delta_{12}+l_{1}\right)\right)} \\
= & \pi^{d / 2} \prod_{i=1}^{l_{1}}\left(\Delta-h_{i}+i-1\right) \frac{\Gamma\left(\Delta-\frac{d}{2}\right) \Gamma\left(\frac{1}{2}\left(\tilde{\Delta}+\Delta_{12}+l_{1}\right)\right) \Gamma\left(\frac{1}{2}\left(\tilde{\Delta}-\Delta_{12}+l_{1}\right)\right)}{\Gamma\left(\tilde{\Delta}+l_{1}\right) \Gamma\left(\frac{1}{2}\left(\Delta+\Delta_{12}+l_{1}\right)\right) \Gamma\left(\frac{1}{2}\left(\Delta-\Delta_{12}+l_{1}\right)\right)} .
\end{aligned}
$$

\section{D.4 Conformal integrals}

We will use the following conformal integral (for $\alpha+\beta+\gamma=d$ )

$$
\begin{aligned}
I_{\alpha, \beta, \gamma}\left(x_{1}, x_{2}, x_{3}\right) & =\int \frac{d^{d} x_{0}}{\left(x_{01}^{2}\right)^{\alpha}\left(x_{02}^{2}\right)^{\beta}\left(x_{03}^{2}\right)^{\gamma}} \\
& =\pi^{d / 2} \frac{\Gamma\left(\frac{d}{2}-\alpha\right) \Gamma\left(\frac{d}{2}-\beta\right) \Gamma\left(\frac{d}{2}-\gamma\right)}{\Gamma(\alpha) \Gamma(\beta) \Gamma(\gamma)}\left(x_{23}^{2}\right)^{\alpha-\frac{d}{2}}\left(x_{13}^{2}\right)^{\beta-\frac{d}{2}}\left(x_{12}^{2}\right)^{\gamma-\frac{d}{2}}
\end{aligned}
$$

to compute the following integral (for $\alpha+\beta+\gamma=d$ )

$$
\begin{aligned}
I_{\alpha, \beta, \gamma}^{n, m}( & \left.x_{1}, x_{2}, x_{3}, z_{31}, z_{32}, z_{2}\right) \\
= & \sum_{k=0}^{n} \frac{n !}{k !(n-k) !}(-1)^{k}\left(x_{23}^{2}\right)^{\frac{n}{2}-k+m}\left(x_{23} \cdot z_{31}\right)^{k} \\
& \quad \frac{d^{d} x_{0}\left(x_{03} \cdot z_{31}\right)^{n-k}\left(x_{03} \cdot z_{32}\right)^{m}\left(x_{02} \cdot z_{2}\right)^{m}}{\left(x_{01}^{2}\right)^{\alpha}\left(x_{02}^{2}\right)^{\beta+\frac{n}{2}+m}\left(x_{03}^{2}\right)^{\gamma+\frac{n}{2}-k+m}+\mathcal{O}\left(\left(z_{2} \cdot z_{32}\right)^{m-1}\right)} \\
= & \sum_{k=0}^{n} \frac{n !}{k !(n-k) !}(-1)^{k}\left(x_{23}^{2}\right)^{\frac{n}{2}-k+m}\left(x_{23} \cdot z_{31}\right)^{k} \frac{\Gamma\left(\gamma-\frac{n}{2}\right)}{2^{n-k+m} \Gamma\left(\gamma+\frac{n}{2}-k+m\right)} \frac{\Gamma\left(\beta+\frac{n}{2}\right)}{2^{m} \Gamma\left(\beta+\frac{n}{2}+m\right)} \\
& \left(z_{2} \cdot \partial_{x_{2}}\right)^{m}\left(z_{32} \cdot \partial_{x_{3}}\right)^{m}\left(z_{31} \cdot \partial_{x_{3}}\right)^{n-k} I_{\alpha, \beta+\frac{n}{2}, \gamma-\frac{n}{2}}\left(x_{1}, x_{2}, x_{3}\right)+\mathcal{O}\left(\left(z_{2} \cdot z_{32}\right)^{m-1}\right) .
\end{aligned}
$$

The computation of the derivative also simplifies significantly when considering only terms of order $\mathcal{O}\left(\left(z_{2} \cdot z_{32}\right)^{m}\right)$. It is enough to consider the contribution of $\left(z_{2} \cdot \partial_{x_{2}}\right)^{m}\left(z_{32} \cdot \partial_{x_{3}}\right)^{m}$ acting on $\left(x_{23}^{2}\right)^{\alpha-h-(n-k-b)}$,

$$
\begin{aligned}
& \left(z_{2} \cdot \partial_{x_{2}}\right)^{m}\left(z_{32} \cdot \partial_{x_{3}}\right)^{m}\left(z_{31} \cdot \partial_{x_{3}}\right)^{n-k} I_{\alpha, \beta+\frac{n}{2}, \gamma-\frac{n}{2}}\left(x_{1}, x_{2}, x_{3}\right)= \\
& \quad \pi^{d / 2} \sum_{b=0}^{n-k} \frac{(n-k) ! m !}{b !(n-k-b) !} 2^{n-k+m} \frac{\Gamma\left(\frac{d}{2}-\alpha+n-k-b+m\right) \Gamma\left(\frac{d}{2}-\beta-\frac{n}{2}+b\right) \Gamma\left(\frac{d}{2}-\gamma+\frac{n}{2}\right)}{\Gamma(\alpha) \Gamma\left(\beta+\frac{n}{2}\right) \Gamma\left(\gamma-\frac{n}{2}\right)} \\
& \quad\left(x_{23}^{2}\right)^{\alpha-\frac{d}{2}-(n-k-b)-m}\left(x_{13}^{2}\right)^{\beta-\frac{d}{2}+\frac{n}{2}-b}\left(x_{12}^{2}\right)^{\gamma-\frac{d}{2}-\frac{n}{2}}\left(x_{13} \cdot z_{31}\right)^{b}\left(x_{23} \cdot z_{31}\right)^{n-k-b}\left(z_{2} \cdot z_{32}\right)^{m} \\
& \quad+\mathcal{O}\left(z_{3 i} \cdot z_{3 j}\right)+\mathcal{O}\left(\left(z_{2} \cdot z_{32}\right)^{m-1}\right) .
\end{aligned}
$$


By inserting this in the last expression for $I_{\alpha, \beta, \gamma}^{n, m}$ in (D.34), one finds

$$
\begin{aligned}
I_{\alpha, \beta, \gamma}^{n, m}( & \left.x_{1}, x_{2}, x_{3}, z_{31}, z_{32}, z_{2}\right) \\
= & \pi^{d / 2} \sum_{k=0}^{n} \sum_{b=0}^{n-k} \frac{n ! m !(-1)^{k}}{k ! b !(n-k-b) ! 2^{m}} \frac{\Gamma\left(\frac{d}{2}-\alpha+n-k-b+m\right) \Gamma\left(\frac{d}{2}-\beta-\frac{n}{2}+b\right) \Gamma\left(\frac{d}{2}-\gamma+\frac{n}{2}\right)}{\Gamma(\alpha) \Gamma\left(\beta+\frac{n}{2}+m\right) \Gamma\left(\gamma+\frac{n}{2}-k+m\right)} \\
& \left(x_{23}^{2}\right)^{\alpha-\frac{d}{2}-\frac{n}{2}+b}\left(x_{13}^{2}\right)^{\beta-\frac{d}{2}+\frac{n}{2}-b}\left(x_{12}^{2}\right)^{\gamma-\frac{d}{2}-\frac{n}{2}}\left(x_{13} \cdot z_{31}\right)^{b}\left(x_{23} \cdot z_{31}\right)^{n-b}\left(z_{2} \cdot z_{32}\right)^{m} \\
& +\mathcal{O}\left(z_{3 i} \cdot z_{3 j}\right)+\mathcal{O}\left(\left(z_{2} \cdot z_{32}\right)^{m-1}\right) \\
= & \pi^{d / 2} \sum_{b=0}^{n} \frac{n ! m !}{b !(n-b) !} \frac{(-1)^{n-b}}{2^{m}} \frac{\Gamma\left(\frac{d}{2}-\alpha+m\right) \Gamma\left(\frac{d}{2}-\beta+\frac{n}{2}\right) \Gamma\left(\frac{d}{2}-\gamma+\frac{n}{2}\right)}{\Gamma(\alpha) \Gamma\left(\beta+\frac{n}{2}+m\right) \Gamma\left(\gamma+\frac{n}{2}+m\right)} \\
& \left(x_{23}^{2}\right)^{\alpha-\frac{d}{2}-\frac{n}{2}+b}\left(x_{13}^{2}\right)^{\beta-\frac{d}{2}+\frac{n}{2}-b}\left(x_{12}^{2}\right)^{\gamma-\frac{d}{2}-\frac{n}{2}}\left(x_{13} \cdot z_{31}\right)^{b}\left(x_{23} \cdot z_{31}\right)^{n-b}\left(z_{2} \cdot z_{32}\right)^{m} \\
& +\mathcal{O}\left(z_{3 i} \cdot z_{3 j}\right)+\mathcal{O}\left(\left(z_{2} \cdot z_{32}\right)^{m-1}\right) \\
= & \pi^{d / 2} \frac{m !}{2^{m}} \frac{\Gamma\left(\frac{d}{2}-\alpha+m\right) \Gamma\left(\frac{d}{2}-\beta+\frac{n}{2}\right) \Gamma\left(\frac{d}{2}-\gamma+\frac{n}{2}\right)}{\Gamma(\alpha) \Gamma\left(\beta+\frac{n}{2}+m\right) \Gamma\left(\gamma+\frac{n}{2}+m\right)} \\
& \left(x_{23}^{2}\right)^{\alpha-\frac{d}{2}}\left(x_{13}^{2}\right)^{\beta-\frac{d}{2}}\left(x_{12}^{2}\right)^{\gamma-\frac{d}{2}}\left(k^{(312)}\left(z_{31}\right)\right)^{n}\left(z_{2} \cdot z_{32}\right)^{m}+\mathcal{O}\left(z_{3 i} \cdot z_{3 j}\right)+\mathcal{O}\left(\left(z_{2} \cdot z_{32}\right)^{m-1}\right) .
\end{aligned}
$$

The sum over $k$ was evaluated using

$$
\sum_{k=0}^{N} \frac{N !}{k !(N-k) !}(-1)^{k} \frac{\Gamma(x-k)}{\Gamma(y-k)}=(-1)^{N} \frac{\Gamma(x-N) \Gamma(y-x+N)}{\Gamma(y) \Gamma(y-x)} .
$$

\section{E OPE limit of conformal blocks in the shadow formalism}

In order for the conformal blocks to satisfy the recursion relation derived above, it is crucial that they have the correct normalization. To compare to other results it is a good idea to consider the OPE limit $x_{12}^{a} \rightarrow 0, x_{34}^{a} \rightarrow 0$. This can be done in physical space by generalizing a trick from [38]. To this end let us work again in physical space, as in the previous appendix. The shadow operator of an operator $\mathcal{O}$ in the irrep $(\Delta, \lambda)$ is given by

$$
\tilde{\mathcal{O}}\left(x_{0}, \mathbf{z}_{0}\right)=\frac{\pi_{\lambda}\left(\mathbf{z}_{0}, \boldsymbol{\partial}_{\mathbf{z}_{4}}\right)}{l_{1} ! l_{2} ! \ldots l_{h_{1}} !} \int \frac{d^{d} x_{4}}{\left(x_{04}^{2}\right)^{d-\Delta}}\left(m^{(04)}\left(z_{41}, \partial_{\bar{z}_{41}}\right)\right)^{l_{1}} \ldots\left(m^{(04)}\left(z_{4 h_{1}}, \partial_{\bar{z}_{4 h_{1}}}\right)\right)^{l_{h_{1}}} \mathcal{O}\left(x_{4}, \overline{\mathbf{z}}_{4}\right) .
$$

For a lighter notation we will choose for the remainder of this appendix to consider Young diagrams with at most three rows $\lambda=\left(l_{1}, l_{2}, l_{3}\right)$. Furthermore, we will compute the OPE limit for conformal blocks with $\lambda_{1}=\lambda_{3}=\bullet$ and $\lambda_{2}=\lambda_{4}=\left(l_{2}, l_{3}\right)$. Other configurations can be treated analogously by replacing some of the vectors $z_{2 i}$ by $z_{1 j}$ or $z_{4 i}$ by $z_{3 j}$.

We want to compute the OPE limit of the conformal partial wave

$$
\begin{aligned}
& W_{\Delta, \lambda}^{\lambda_{1} \lambda_{2} \lambda_{3} \lambda_{4}}= \\
& \quad \frac{1}{\mathcal{S}_{\Delta \Delta_{34}}^{\lambda} \mathcal{S}_{\tilde{\Delta} \Delta_{34}}^{\lambda}} \int d^{d} x_{0}\left\langle\mathcal{O}_{1}\left(x_{1}\right) \mathcal{O}_{2}\left(x_{2}, \mathbf{z}_{2}\right) \mathcal{O}\left(x_{0}, \boldsymbol{\partial}_{\mathbf{z}_{0}}\right)\right\rangle\left\langle\mathcal{O}_{3}\left(x_{3}\right) \mathcal{O}_{4}\left(x_{4}, \mathbf{z}_{4}\right) \tilde{\mathcal{O}}\left(x_{0}, \mathbf{z}_{0}\right)\right\rangle,
\end{aligned}
$$


where the three-point functions are given by ${ }^{7}$

$$
\begin{aligned}
& \left\langle\mathcal{O}_{1}\left(x_{1}\right) \mathcal{O}_{2}\left(x_{2}, \mathbf{z}_{2}\right) \mathcal{O}\left(x_{0}, \mathbf{z}_{0}\right)\right\rangle= \\
& \pi_{\lambda}\left(\mathbf{z}_{0}, \boldsymbol{\partial}_{\overline{\mathbf{z}}_{0}}\right) \frac{\left(k^{(012)}\left(\bar{z}_{01}\right)\right)^{l_{1}}\left(m^{(20)}\left(z_{21}, \bar{z}_{02}\right)\right)^{l_{2}}\left(m^{(20)}\left(z_{22}, \bar{z}_{03}\right)\right)^{l_{3}}}{\left(x_{12}^{2}\right)^{\frac{\Delta_{1}+\Delta_{2}-\Delta}{2}}\left(x_{01}^{2}\right)^{\frac{\Delta+\Delta_{1}-\Delta_{2}}{2}}\left(x_{02}^{2}\right)^{\frac{\Delta+\Delta_{2}-\Delta_{1}}{2}}} .
\end{aligned}
$$

To perform the OPE limit one can use

$$
k^{(012)}\left(z_{01}\right) \underset{x_{12} \rightarrow 0}{\sim} \frac{1}{\sqrt{x_{12}^{2}}} m^{(20)}\left(x_{12}, z_{01}\right),
$$

and hence

$$
\begin{aligned}
& \left\langle\mathcal{O}_{1}\left(x_{1}\right) \mathcal{O}_{2}\left(x_{2}, \mathbf{z}_{2}\right) \mathcal{O}\left(x_{0}, \mathbf{z}_{0}\right)\right\rangle \underset{x_{12} \rightarrow 0}{\sim} \\
& \quad \pi_{\lambda}\left(\mathbf{z}_{0}, \boldsymbol{\partial}_{\overline{\mathbf{z}}_{0}}\right) \frac{\left(m^{(20)}\left(x_{12}, \bar{z}_{01}\right)\right)^{l_{1}}\left(m^{(20)}\left(z_{21}, \bar{z}_{02}\right)\right)^{l_{2}}\left(m^{(20)}\left(z_{22}, \bar{z}_{03}\right)\right)^{l_{3}}}{\left(x_{12}^{2}\right)^{\frac{\Delta_{1}+\Delta_{2}-\Delta+l_{1}}{2}}\left(x_{02}^{2}\right)^{\Delta}} .
\end{aligned}
$$

Inserting this into (E.2), and using the definition of the shadow operator (E.1), one finds

$$
\begin{aligned}
W_{\Delta, \lambda}^{\lambda_{1} \lambda_{2} \lambda_{3} \lambda_{4}} \underset{x_{12}}{\sim} \sim 0 & \frac{1}{\mathcal{S}_{\Delta \Delta_{34}}^{\lambda} \mathcal{S}_{\tilde{\Delta} \Delta_{34}}^{\lambda}}\left(x_{12}^{2}\right)^{\frac{\Delta-l_{1}-\Delta_{1}-\Delta_{2}}{2}}\left\langle\mathcal{O}_{3}\left(x_{3}\right) \mathcal{O}_{4}\left(x_{4}, \mathbf{z}_{4}\right) \tilde{\tilde{\mathcal{O}}}\left(x_{2}, x_{12}, z_{21}, z_{22}\right)\right\rangle \\
& =\left(x_{12}^{2}\right)^{\frac{\Delta-l_{1}-\Delta_{1}-\Delta_{2}}{2}}\left\langle\mathcal{O}_{3}\left(x_{3}\right) \mathcal{O}_{4}\left(x_{4}, \mathbf{z}_{4}\right) \mathcal{O}\left(x_{2}, x_{12}, z_{21}, z_{22}\right)\right\rangle .
\end{aligned}
$$

Doing the limit $x_{34}^{a} \rightarrow 0$ in a similar way leads to

$$
W_{\Delta, \lambda}^{\lambda_{1} \lambda_{2} \lambda_{3} \lambda_{4}} \underset{\substack{x_{12} \rightarrow 0 \\ x_{34} \rightarrow 0}}{\sim} \pi_{\lambda}\left(x_{12}, z_{21}, z_{22}, \boldsymbol{\partial}_{\mathbf{z}_{0}}\right) \frac{\left(m^{(24)}\left(z_{01}, x_{34}\right)\right)^{l_{1}}\left(m^{(24)}\left(z_{02}, z_{41}\right)\right)^{l_{2}}\left(m^{(24)}\left(z_{03}, z_{42}\right)\right)^{l_{3}}}{\left(x_{24}^{2}\right)^{\Delta}\left(x_{12}^{2}\right)^{\frac{1}{2}\left(\Delta_{1}+\Delta_{2}-\Delta+l_{1}\right)}\left(x_{34}^{2}\right)^{\frac{1}{2}\left(\Delta_{3}+\Delta_{4}-\Delta+l_{1}\right)}} .
$$

In order to extract the normalization it is convenient to consider only the term which is of leading order in the building blocks $m^{(24)}\left(x_{12}, x_{34}\right), m^{(24)}\left(z_{21}, x_{41}\right)$ and $m^{(24)}\left(z_{22}, x_{42}\right)$. This term has a prefactor that can be found by considering the birdtrack diagram in (2.58) and removing all antisymmetrizations, resulting in a factor of

$$
\frac{n_{\lambda}}{\prod_{i=1}^{l_{1}} h_{i} !}
$$

Finally one can use that

$$
1-v \underset{\substack{x_{12} \rightarrow 0 \\ x_{34} \rightarrow 0}}{\sim} \frac{2 m^{(24)}\left(x_{12}, x_{34}\right)}{x_{24}^{2}}
$$

and find

$$
\begin{aligned}
& W_{\Delta, \lambda}^{\lambda_{1} \lambda_{2} \lambda_{3} \lambda_{4}} \underset{\underset{x_{12} \rightarrow 0}{\sim} \underset{x_{34} \rightarrow 0}{\sim}}{\sim} \frac{n_{\lambda}}{\prod_{i=1}^{l_{1}} h_{i} !} 2^{-l_{1}} u^{\frac{1}{2}\left(\Delta-l_{1}\right)}(1-v)^{l_{1}} \frac{\left(m^{(24)}\left(z_{21}, z_{41}\right)\right)^{l_{2}}\left(m^{(24)}\left(z_{22}, z_{42}\right)\right)^{l_{3}}}{\left(x_{12}^{2}\right)^{\frac{\Delta_{1}+\Delta_{2}}{2}}\left(x_{34}^{2}\right)^{\frac{\Delta_{3}+\Delta_{4}}{2}}} \\
&+\mathcal{O}\left(\left(\frac{1-v}{\sqrt{u}}\right)^{l_{1}-1},\left(m^{(24)}\left(z_{21}, z_{41}\right)\right)^{l_{2}-1},\left(m^{(24)}\left(z_{22}, z_{42}\right)\right)^{l_{3}-1}\right) .
\end{aligned}
$$

\footnotetext{
${ }^{7}$ We are omitting the projector $\pi_{\lambda_{2}}$ which has no impact on this computation.
} 


\section{F Spherical tensor harmonics}

In this appendix the relation between projectors to $\mathrm{SO}(d)$ irreps and spherical tensor harmonics on the sphere $S^{d-1}$ is explained. In the case of projectors to traceless symmetric tensors this relation is just the fact that the projectors are encoded by Gegenbauer polynomials (2.7), which are scalar spherical harmonics. In the radial coordinates of [49], the conformal blocks are naturally written in terms of spherical tensor harmonics [51].

Consider a tensor field on $S^{d-1} \in \mathbb{R}^{d}$. We shall work in the embedding space $\mathbb{R}^{d}$ and impose transversality

$$
x^{a_{i}} t_{a_{1} \ldots a_{k}}(x)=0,
$$

for all $i=1, \ldots, k$ and $x_{a} x^{a}=1$. As shown in subsection F.1 below, covariant derivatives on the sphere are just partial derivatives $\partial_{a}=\frac{\partial}{\partial x^{a}}$ on $\mathbb{R}^{d}$ projected to the sphere (over all indices of the resulting tensor). Therefore, the laplacian on the sphere can be written as

$$
\nabla^{2} t_{a_{1} \ldots a_{k}}=P^{a b} \partial_{a}\left(P_{b}^{c} P_{b_{1}}^{c_{1}} \ldots P_{b_{k}}^{c_{k}} \partial_{c} t_{c_{1} \ldots c_{k}}\right) P_{a_{1}}^{b_{1}} \ldots P_{a_{k}}^{b_{k}},
$$

where $P_{a b}=\delta_{a b}-x_{a} x_{b}$ is a projector onto the unit sphere. Using (F.1) on the sphere $x_{a} x^{a}=1$, one can simplify this expression to

$$
\nabla^{2} t_{a_{1} \ldots a_{k}}=P_{a_{1}}^{b_{1}} \ldots P_{a_{k}}^{b_{k}}\left(\partial_{a} \partial^{a}-x^{a} x^{b} \partial_{a} \partial_{b}-(d-1) x^{a} \partial_{a}+k\right) t_{b_{1} \ldots b_{k}}
$$

Another interesting differential operator to consider is the quadratic Casimir of the symmetry group $\mathrm{SO}(d)$, generated by

$$
J_{a b}=i\left(x_{a} \partial_{b}-x_{b} \partial_{a}\right)+\Sigma_{a b},
$$

where $\Sigma_{a b}$ rotates the indices of a tensor. More precisely,

$$
\Sigma_{a b} t_{a_{1} \ldots a_{k}}=\sum_{i=1}^{k}\left[M_{a b}\right]_{a_{i}}^{c} t_{a_{1} \ldots a_{i-1} c a_{i+1} \ldots a_{k}},
$$

with $\left[M^{a b}\right]_{e}^{c}=i\left(\delta_{e}^{a} \delta^{b c}-\delta_{e}^{b} \delta^{a c}\right)$. The quadratic Casimir is then given by

$$
\frac{1}{2} J_{a b} J^{a b}=-x^{a} \partial^{b}\left(x_{a} \partial_{b}-x_{b} \partial_{a}\right)+2 i x_{a} \partial_{b} \Sigma^{a b}+\frac{1}{2} \Sigma_{a b} \Sigma^{a b} .
$$

Acting on a tensor obeying (F.1) on the sphere $x_{a} x^{a}=1$, it gives

$$
\frac{1}{2} J_{a b} J^{a b} t_{a_{1} \ldots a_{k}}=P_{a_{1}}^{b_{1}} \ldots P_{a_{k}}^{b_{k}}\left[-\nabla^{2}-k+\frac{1}{2} \Sigma_{a b} \Sigma^{a b}\right] t_{b_{1} \ldots b_{k}},
$$

where we used expression (F.3) for the laplacian on the sphere.

Now consider a tensor field defined by the following contraction with a traceless mixedsymmetry tensor

$$
t_{\left(b_{1} \ldots b_{l_{2}}\right)\left(c_{1} \ldots c_{l_{3}}\right) \ldots}=x^{e_{1}} \ldots x^{e_{l_{1}}} f_{\left(e_{1} \ldots e_{l_{1}}\right)\left(b_{1} \ldots b_{l_{2}}\right)\left(c_{1} \ldots c_{l_{3}}\right) \ldots} .
$$


Notice that tracelessness and property (2.10) of $f$ implies transversality (F.1) and

$$
\nabla^{a_{i}} t_{a_{1} \ldots a_{k}}=0
$$

where $k=\sum_{i=2} l_{i}$ and $a_{i}$ can be any of the indices of the tensor. Using formula (F.3) it is easy to obtain

$$
\nabla^{2} t_{a_{1} \ldots a_{k}}=-\left[l_{1}\left(l_{1}+d-2\right)-k\right] t_{a_{1} \ldots a_{k}} .
$$

Moreover,

$$
\frac{1}{2} \Sigma_{a b} \Sigma^{a b} t_{\left(b_{1} \ldots b_{l_{2}}\right)\left(c_{1} \ldots c_{l_{3}}\right) \ldots}=\left[\sum_{i=1}^{h_{1}-1} l_{i+1}\left(l_{i+1}+d-2 i\right)\right] t_{\left(b_{1} \ldots b_{l_{2}}\right)\left(c_{1} \ldots c_{l_{3}}\right) \ldots},
$$

because $\Sigma_{a b}$ only rotates the indices, and therefore $\frac{1}{2} \Sigma_{a b} \Sigma^{a b}$ just measures the Casimir of the irreducible tensor. This statement can also be checked explicitly using the definition (F.5). One can also check that (F.7) leads to the expected result

$$
\frac{1}{2} J_{a b} J^{a b} t_{\left(b_{1} \ldots b_{l_{2}}\right)\left(c_{1} \ldots c_{l_{3}}\right) \ldots}=\left[\sum_{i=1}^{h_{1}} l_{i}\left(l_{i}+d-2 i\right)\right] t_{\left(b_{1} \ldots b_{l_{2}}\right)\left(c_{1} \ldots c_{l_{3}}\right) \ldots} .
$$

Using the projectors of the previous sections, we can construct the following function

$$
\Omega_{\lambda}^{b_{1} \ldots, b_{1}^{\prime} \ldots}(x, y)=c_{\lambda} x_{a_{1}} \ldots x_{a_{l_{1}}} \pi_{\lambda}^{\left(a_{1} \ldots a_{l_{1}}\right)\left(b_{1} \ldots b_{l_{2}}\right) \ldots,\left(a_{1}^{\prime} \ldots a_{l_{1}}^{\prime}\right)\left(b_{1}^{\prime} \ldots b_{l_{2}}^{\prime}\right) \ldots} y_{a_{1}^{\prime}} \ldots y_{a_{l_{1}}^{\prime}} .
$$

The arguments above show that this function is a tensor harmonic at the point $x$ with indices $b_{1} \ldots$ It is also a tensor harmonic at point $y$ with indices $b_{1}^{\prime} \ldots$ We shall fix the normalization constant $c_{\lambda}$ by imposing the following orthogonality relation

$$
\int_{S^{d-1}} d y \Omega_{\lambda}^{a_{1} \ldots, b_{1} \cdots}(x, y) \delta_{b_{1} b_{1}^{\prime}} \ldots \Omega_{\lambda^{\prime}}^{b_{1}^{\prime} \ldots, c_{1} \cdots}(y, z)=\delta_{\lambda, \lambda^{\prime}} \Omega_{\lambda}^{a_{1} \ldots, c_{1} \cdots}(x, z) .
$$

Using the definition (F.13) the only integral that we need to compute is

$$
I^{a_{1} \ldots a_{k}}=\int_{S^{d-1}} d y y^{a_{1}} \ldots y^{a_{k}} .
$$

Rotational and permutation symmetry imply that $I^{a_{1} \ldots a_{k}}=0$ for odd $k$ and

$$
I^{a_{1} \ldots a_{2 k}}=q_{k} \delta^{\left(a_{1} a_{2}\right.} \ldots \delta^{\left.a_{2 k-1} a_{2 k}\right)},
$$

for some constant $q_{k}$. This constant can be determined by computing the full contraction ${ }^{8}$

$$
\delta_{a_{1} a_{2}} \ldots \delta_{a_{2 k-1} a_{2 k}} I^{a_{1} \ldots a_{2 k}}=q_{k} 4^{k} k !\left(\frac{d}{2}\right)_{k}=\operatorname{Vol}\left(S^{d-1}\right) .
$$

This is sufficient to verify the orthogonality relation (F.14) and to determine the normalization constant

$$
c_{\lambda}=\frac{1}{l_{1} ! q_{l_{1}}}=\frac{4^{l_{1}}\left(\frac{d}{2}\right)_{l_{1}}}{\operatorname{Vol}\left(S^{d-1}\right)} .
$$

\footnotetext{
${ }^{8}$ The full contraction of $k$ Kronecker-deltas with the total index symmetrization of another set of $k$ Kronecker-deltas can be determined recursively.
} 
Let us now consider again the orthogonality relation (F.14) in the case of scalar harmonics and let us sum over $l$,

$$
\int_{S^{d-1}} d y \Omega_{\left(l^{\prime}\right)}(x, y) \sum_{l=0}^{\infty} \Omega_{(l)}(y, z)=\Omega_{\left(l^{\prime}\right)}(x, z) .
$$

This suggests the following completeness relation

$$
\sum_{l=0}^{\infty} \Omega_{(l)}(y, z)=\delta(y, z),
$$

where the delta-function is defined with respect to the sphere metric. This reasoning can be generalized to the case of tensor harmonics. We illustrate this method in the case of vector harmonics, corresponding to $\lambda=\left(l_{1}, 1\right)$. Summing the orthogonality relation (F.14) over $l_{1}$ we find

$$
\int_{S^{d-1}} d y \Omega_{\left(l_{1}^{\prime}, 1\right)}^{a, b^{\prime}}(x, y) \delta_{b^{\prime} b} \sum_{l_{1}=1}^{\infty} \Omega_{\left(l_{1}, 1\right)}^{b, c}(y, z)=\Omega_{\left(l_{1}^{\prime}, 1\right)}^{a, c}(x, z),
$$

which leads to

$$
\sum_{l_{1}=1}^{\infty} \Omega_{\left(l_{1}, 1\right)}^{b, c}(y, z)+\nabla_{y}^{b} \nabla_{z}^{c} Q(y, z)=\delta^{b c} \delta(x, z),
$$

where $Q(y, z)$ can be any function of $y \cdot z$ because its contribution to (F.21) vanishes by integration by parts. To determine this function we act with $\delta_{b^{\prime} b} \nabla_{y}^{b^{\prime}}$ on the last equation,

$$
\nabla_{z}^{c} \nabla_{y}^{2} Q(y, z)=\nabla_{y}^{c} \delta(x, z)=-\nabla_{z}^{c} \delta(x, z) .
$$

Comparing with (F.20) and using the laplacian eigenvalues of the scalar harmonics we conclude that

$$
Q(y, z)=\sum_{l=0}^{\infty} \frac{1}{l(l+d-2)} \Omega_{(l)}(y, z) .
$$

The discussion here is very similar to the discussion of harmonic functions in AdS in [54].

\section{F.1 Covariant derivatives}

We consider a tensor $t_{a_{1} \ldots a_{k}}$ obeying (F.1). Let us denote by $y^{\alpha}$ a set of $(d-1)$-coordinates parametrizing the unit sphere $S^{d-1}$. In these coordinates, the tensor is given by

$$
\tilde{t}_{\alpha_{1} \ldots \alpha_{k}}=\frac{\partial x^{a_{1}}}{\partial y^{\alpha_{1}}} \cdots \frac{\partial x^{a_{k}}}{\partial y^{\alpha_{k}}} t_{a_{1} \ldots a_{k}},
$$

and its covariant derivative is

$$
\begin{aligned}
\nabla_{\beta} \tilde{t}_{\alpha_{1} \ldots \alpha_{k}}= & \frac{\partial}{\partial y^{\beta}} \tilde{t}_{\alpha_{1} \ldots \alpha_{k}}-\sum_{i=1}^{k} \Gamma_{\beta \alpha_{i}}^{\gamma} \tilde{t}_{\alpha_{1} \ldots \alpha_{i-1} \gamma \alpha_{i+1} \ldots \alpha_{k}} \\
= & \frac{\partial x^{b}}{\partial y^{\beta}} \frac{\partial x^{a_{1}}}{\partial y^{\alpha_{1}}} \ldots \frac{\partial x^{a_{k}}}{\partial y^{\alpha_{k}}} \frac{\partial}{\partial x^{b}} t_{a_{1} \ldots a_{k}} \\
& +\sum_{i=1}^{k}\left(\frac{\partial^{2} x^{a_{i}}}{\partial y^{\beta} \partial y^{\alpha_{i}}}-\Gamma_{\beta \alpha_{i}}^{\gamma} \frac{\partial x^{a_{i}}}{\partial y^{\gamma}}\right) \frac{\partial x^{a_{1}}}{\partial y^{\alpha_{1}}} \ldots \frac{\partial x^{a_{i-1}}}{\partial y^{\alpha_{i-1}}} \frac{\partial x^{a_{i+1}}}{\partial y^{\alpha_{i+1}}} \ldots \frac{\partial x^{a_{k}}}{\partial y^{\alpha_{k}}} t_{a_{1} \ldots a_{k}}
\end{aligned}
$$


We will now show that the last line vanishes and therefore covariant derivatives on the sphere are equal to partial derivatives on $\mathbb{R}^{d}$ projected to the sphere. It is sufficient to show that

$$
\frac{\partial^{2} x^{a}}{\partial y^{\beta} \partial y^{\alpha}}-\Gamma_{\beta \alpha}^{\gamma} \frac{\partial x^{a}}{\partial y^{\gamma}} \propto x^{a},
$$

because the last line in (F.26) vanishes due to the transversality condition (F.1). Equation (F.27) is equivalent to

$$
\frac{\partial^{2} x^{a}}{\partial y^{\beta} \partial y^{\alpha}} \frac{\partial x_{a}}{\partial y^{\mu}}-\Gamma_{\beta \alpha}^{\gamma} \frac{\partial x^{a}}{\partial y^{\gamma}} \frac{\partial x_{a}}{\partial y^{\mu}}=0,
$$

which can be easily verified using the sphere metric

$$
g_{\alpha \beta}=\frac{\partial x^{a}}{\partial y^{\alpha}} \frac{\partial x_{a}}{\partial y^{\beta}},
$$

and the expression for the Levi-Civita connection

$$
\Gamma_{\beta \alpha}^{\gamma}=\frac{1}{2} g^{\gamma \mu}\left(g_{\mu \beta, \alpha}+g_{\mu \alpha, \beta}-g_{\beta \alpha, \mu}\right) .
$$

Open Access. This article is distributed under the terms of the Creative Commons Attribution License (CC-BY 4.0), which permits any use, distribution and reproduction in any medium, provided the original author(s) and source are credited.

\section{References}

[1] S. Ferrara, A.F. Grillo and R. Gatto, Tensor representations of conformal algebra and conformally covariant operator product expansion, Annals Phys. 76 (1973) 161 [INSPIRE].

[2] A.M. Polyakov, Nonhamiltonian approach to conformal quantum field theory, Zh. Eksp. Teor. Fiz. 66 (1974) 23 [INSPIRE].

[3] R. Rattazzi, V.S. Rychkov, E. Tonni and A. Vichi, Bounding scalar operator dimensions in 4D CFT, JHEP 12 (2008) 031 [arXiv:0807.0004] [INSPIRE].

[4] V.S. Rychkov and A. Vichi, Universal constraints on conformal operator dimensions, Phys. Rev. D 80 (2009) 045006 [arXiv: 0905.2211] [inSPIRE].

[5] D. Poland and D. Simmons-Duffin, Bounds on $4 D$ conformal and superconformal field theories, JHEP 05 (2011) 017 [arXiv: 1009.2087] [INSPIRE].

[6] R. Rattazzi, S. Rychkov and A. Vichi, Central charge bounds in $4 D$ conformal field theory, Phys. Rev. D 83 (2011) 046011 [arXiv: 1009.2725] [InSPIRE].

[7] R. Rattazzi, S. Rychkov and A. Vichi, Bounds in $4 D$ conformal field theories with global symmetry, J. Phys. A 44 (2011) 035402 [arXiv:1009.5985] [InSPIRE].

[8] D. Poland, D. Simmons-Duffin and A. Vichi, Carving out the space of $4 D$ CFTs, JHEP 05 (2012) 110 [arXiv:1109.5176] [INSPIRE].

[9] S. El-Showk, M.F. Paulos, D. Poland, S. Rychkov, D. Simmons-Duffin and A. Vichi, Solving the 3D Ising model with the conformal bootstrap, Phys. Rev. D 86 (2012) 025022 [arXiv: 1203.6064] [INSPIRE]. 
[10] P. Liendo, L. Rastelli and B.C. van Rees, The bootstrap program for boundary $C F T_{d}$, JHEP 07 (2013) 113 [arXiv: 1210.4258] [INSPIRE].

[11] C. Beem, L. Rastelli and B.C. van Rees, The $\mathcal{N}=4$ superconformal bootstrap, Phys. Rev. Lett. 111 (2013) 071601 [arXiv:1304.1803] [InSPIRE].

[12] F. Gliozzi, More constraining conformal bootstrap, Phys. Rev. Lett. 111 (2013) 161602 [arXiv:1307.3111] [INSPIRE].

[13] F. Kos, D. Poland and D. Simmons-Duffin, Bootstrapping the $O(N)$ vector models, JHEP 06 (2014) 091 [arXiv: 1307.6856] [INSPIRE].

[14] S. El-Showk, M. Paulos, D. Poland, S. Rychkov, D. Simmons-Duffin and A. Vichi, Conformal field theories in fractional dimensions, Phys. Rev. Lett. 112 (2014) 141601 [arXiv: 1309.5089] [INSPIRE].

[15] S. El-Showk, M.F. Paulos, D. Poland, S. Rychkov, D. Simmons-Duffin and A. Vichi, Solving the $3 d$ Ising model with the conformal bootstrap II. c-Minimization and precise critical exponents, J. Stat. Phys. 157 (2014) 869 [arXiv:1403.4545] [INSPIRE].

[16] Y. Nakayama and T. Ohtsuki, Approaching the conformal window of $O(n) \times O(m)$ symmetric Landau-Ginzburg models using the conformal bootstrap, Phys. Rev. D 89 (2014) 126009 [arXiv: 1404.0489] [INSPIRE].

[17] S.M. Chester, J. Lee, S.S. Pufu and R. Yacoby, The $\mathcal{N}=8$ superconformal bootstrap in three dimensions, JHEP 09 (2014) 143 [arXiv: 1406.4814] [INSPIRE].

[18] F. Kos, D. Poland and D. Simmons-Duffin, Bootstrapping mixed correlators in the 3D Ising model, JHEP 11 (2014) 109 [arXiv: 1406.4858] [INSPIRE].

[19] M.F. Paulos, JuliBootS: a hands-on guide to the conformal bootstrap, arXiv:1412.4127 [INSPIRE].

[20] C. Beem, M. Lemos, P. Liendo, L. Rastelli and B.C. van Rees, The $\mathcal{N}=2$ superconformal bootstrap, JHEP 03 (2016) 183 [arXiv:1412.7541] [INSPIRE].

[21] D. Simmons-Duffin, A semidefinite program solver for the conformal bootstrap, JHEP 06 (2015) 174 [arXiv: 1502.02033] [INSPIRE].

[22] F. Gliozzi, P. Liendo, M. Meineri and A. Rago, Boundary and interface CFTs from the conformal bootstrap, JHEP 05 (2015) 036 [arXiv:1502.07217] [INSPIRE].

[23] N. Bobev, S. El-Showk, D. Mazac and M.F. Paulos, Bootstrapping SCFTs with four supercharges, JHEP 08 (2015) 142 [arXiv:1503.02081] [INSPIRE].

[24] F. Kos, D. Poland, D. Simmons-Duffin and A. Vichi, Bootstrapping the $O(N)$ archipelago, JHEP 11 (2015) 106 [arXiv: 1504.07997] [INSPIRE].

[25] C. Beem, M. Lemos, L. Rastelli and B.C. van Rees, The $(2,0)$ superconformal bootstrap, Phys. Rev. D 93 (2016) 025016 [arXiv:1507.05637] [INSPIRE].

[26] L. Iliesiu, F. Kos, D. Poland, S.S. Pufu, D. Simmons-Duffin and R. Yacoby, Bootstrapping $3 D$ fermions, JHEP 03 (2016) 120 [arXiv:1508.00012] [INSPIRE].

[27] M. Lemos and P. Liendo, Bootstrapping $\mathcal{N}=2$ chiral correlators, JHEP 01 (2016) 025 [arXiv: 1510.03866] [INSPIRE].

[28] Y.-H. Lin, S.-H. Shao, D. Simmons-Duffin, Y. Wang and X. Yin, $\mathcal{N}=4$ superconformal bootstrap of the K3 CFT, arXiv:1511.04065 [INSPIRE]. 
[29] S.M. Chester, L.V. Iliesiu, S.S. Pufu and R. Yacoby, Bootstrapping $O(N)$ vector models with four supercharges in $3 \leq d \leq 4$, arXiv: 1511.07552 [INSPIRE].

[30] D. Li, D. Meltzer and D. Poland, Conformal collider physics from the lightcone bootstrap, JHEP 02 (2016) 143 [arXiv: 1511.08025] [INSPIRE].

[31] S.M. Chester and S.S. Pufu, Towards bootstrapping $Q E D_{3}$, arXiv:1601.03476 [INSPIRE].

[32] C. Behan, PyCFTBoot: a flexible interface for the conformal bootstrap, arXiv:1602.02810 [INSPIRE].

[33] D.M. Hofman, D. Li, D. Meltzer, D. Poland and F. Rejon-Barrera, A proof of the conformal collider bounds, JHEP 06 (2016) 111 [arXiv:1603.03771] [INSPIRE].

[34] F. Kos, D. Poland, D. Simmons-Duffin and A. Vichi, Precision islands in the Ising and $O(N)$ models, arXiv: 1603.04436 [INSPIRE].

[35] M.S. Costa, J. Penedones, D. Poland and S. Rychkov, Spinning conformal blocks, JHEP 11 (2011) 154 [arXiv:1109.6321] [InSPIRE].

[36] A.C. Echeverri, E. Elkhidir, D. Karateev and M. Serone, Deconstructing conformal blocks in $4 D C F T, J H E P 08$ (2015) 101 [arXiv:1505.03750] [INSPIRE].

[37] S. Ferrara, A. Grillo, G. Parisi and R. Gatto, The shadow operator formalism for conformal algebra. Vacuum expectation values and operator products, Lett. Nuovo Cim. 4 (1972) 115.

[38] F.A. Dolan and H. Osborn, Conformal partial waves: further mathematical results, arXiv:1108.6194 [INSPIRE].

[39] D. Simmons-Duffin, Projectors, shadows and conformal blocks, JHEP 04 (2014) 146 [arXiv: 1204.3894] [INSPIRE].

[40] F.A. Dolan and H. Osborn, Conformal four point functions and the operator product expansion, Nucl. Phys. B 599 (2001) 459 [hep-th/0011040] [InSPIRE].

[41] F.A. Dolan and H. Osborn, Conformal partial waves and the operator product expansion, Nucl. Phys. B 678 (2004) 491 [hep-th/0309180] [INSPIRE].

[42] F. Rejon-Barrera and D. Robbins, Scalar-vector bootstrap, JHEP 01 (2016) 139 [arXiv: 1508.02676] [INSPIRE].

[43] B. Geyer and M. Lazar, Twist decomposition of nonlocal light-cone operators II: general tensors of 2nd rank, Nucl. Phys. B 581 (2000) 341 [hep-th/0003080] [INSPIRE].

[44] J. Eilers, Geometric twist decomposition off the light-cone for nonlocal QCD operators, hep-th/0608173 [INSPIRE].

[45] M.S. Costa and T. Hansen, Conformal correlators of mixed-symmetry tensors, JHEP 02 (2015) 151 [arXiv:1411.7351] [INSPIRE].

[46] P. Cvitanović, Group theory: Birdtracks, Lie's and exceptional groups, Princeton University Press, Princeton U.S.A. (2008).

[47] M.S. Costa, J. Penedones, D. Poland and S. Rychkov, Spinning conformal correlators, JHEP 11 (2011) 071 [arXiv: 1107.3554] [INSPIRE].

[48] A.C. Echeverri, E. Elkhidir, D. Karateev and M. Serone, Seed conformal blocks in $4 D C F T$, arXiv:1601.05325 [INSPIRE].

[49] M. Hogervorst and S. Rychkov, Radial coordinates for conformal blocks, Phys. Rev. D 87 (2013) 106004 [arXiv:1303.1111] [INSPIRE]. 
[50] J. Penedones, E. Trevisani and M. Yamazaki, Recursion relations for conformal blocks, arXiv: 1509.00428 [INSPIRE].

[51] M.S. Costa, T. Hansen, J. Penedones and E. Trevisani, Radial expansion for spinning conformal blocks, arXiv:1603.05552 [INSPIRE].

[52] V.K. Dobrev, V.B. Petkova, S.G. Petrova and I.T. Todorov, Dynamical derivation of vacuum operator product expansion in Euclidean conformal quantum field theory, Phys. Rev. D 13 (1976) 887 [INSPIRE].

[53] N. El Samra and R.C. King, Dimensions of irreducible representations of the classical Lie groups, J. Phys. A 12 (1979) 2317.

[54] M.S. Costa, V. Gonçalves and J. Penedones, Spinning AdS propagators, JHEP 09 (2014) 064 [arXiv: 1404.5625] [INSPIRE]. 